\title{
Linear, Low Noise Microwave Photonic Systems using Phase and Frequency Modulation
}

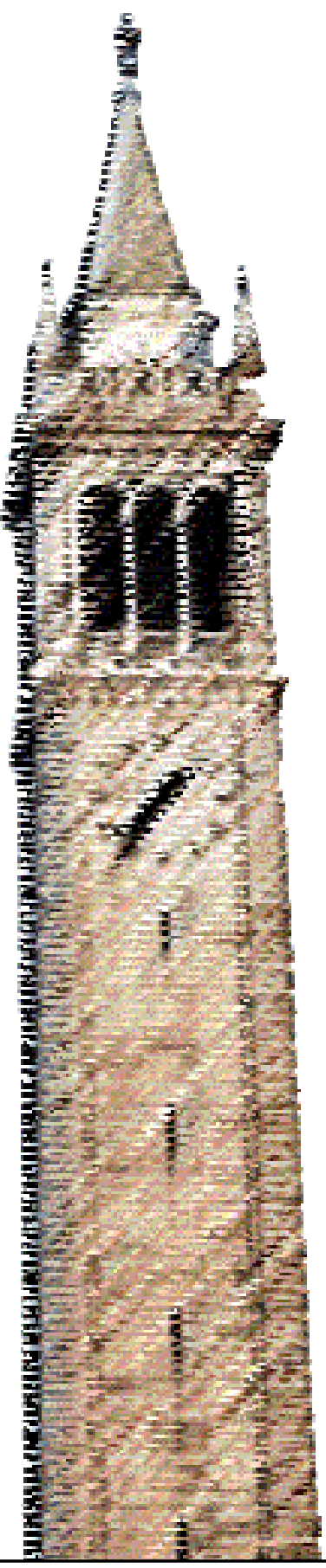

John Wyrwas

Electrical Engineering and Computer Sciences University of California at Berkeley

Technical Report No. UCB/EECS-2012-89

http://www.eecs.berkeley.edu/Pubs/TechRpts/2012/EECS-2012-89.html

May 11, 2012 
Copyright (C) 2012, by the author(s).

All rights reserved.

Permission to make digital or hard copies of all or part of this work for personal or classroom use is granted without fee provided that copies are not made or distributed for profit or commercial advantage and that copies bear this notice and the full citation on the first page. To copy otherwise, to republish, to post on servers or to redistribute to lists, requires prior specific permission. 
Linear, Low Noise Microwave Photonic Systems using Phase and Frequency Modulation

\author{
by \\ John Michael Wyrwas \\ A dissertation submitted in partial satisfaction \\ of the requirements for the degree of \\ Doctor of Philosophy \\ in \\ Engineering - Electrical Engineering and Computer Sciences \\ in the \\ GRADUATE DIVISION \\ of the \\ UNIVERSITY OF CALIFORNIA, BERKELEY \\ Committee in charge: \\ Professor Ming C. Wu, Chair \\ Professor Constance Chang-Hasnain \\ Professor Xiang Zhang
}

Spring 2012 



\begin{abstract}
Linear, Low Noise Microwave Photonic Systems using Phase and Frequency Modulation

by

John Michael Wyrwas

Doctor of Philosophy in Engineering - Electrical Engineering and Computer Sciences

University of California, Berkeley

Ming C. Wu, Chair

Photonic systems that transmit and process microwave-frequency analog signals have traditionally been encumbered by relatively large signal distortion and noise. Optical phase modulation (PM) and frequency modulation (FM) are promising techniques that can improve system performance. In this dissertation, I discuss an optical filtering approach to demodulation of PM and FM signals, which does not rely on high frequency electronics, and which scales in linearity with increasing photonic integration. I present an analytical model, filter designs and simulations, and experimental results using planar lightwave circuit (PLC) filters and FM distributed Bragg reflector (DBR) lasers. The linearity of the PM and FM photonic links exceed that of the current state-of-the-art.
\end{abstract}

Ming C. Wu
Dissertation Committee Chair 


\section{Contents}

1 Introduction $\quad 1$

1.1 Microwave photonics applications . . . . . . . . . . . . . 1

1.2 Advantages for signal distribution . . . . . . . . . . . . . . . 3

1.3 Dynamic range challenges . . . . . . . . . . . . . . . . . 3

1.3.1 Microwave photonic links . . . . . . . . . . . . . . . 4

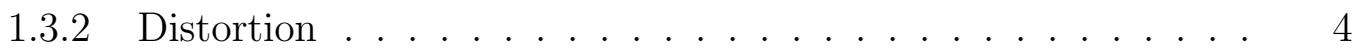

1.3 .3 Noise . . . . . . . . . . . . . . . . . 6

1.3.4 System example . . . . . . . . . . . . . . . . 7

1.4 Techniques to improve dynamic range . . . . . . . . . . . . . 9

2 Theory of PM-DD and FM-DD links 11

2.1 Motivation for phase and frequency modulation . . . . . . . . . 11

2.2 Link architecture . . . . . . . . . . . . . . . . . . . 12

2.3 History . . . . . . . . . . . . . . . . . . . . . . . . . . . . . . . . . . .

2.4 Analytical link analysis . . . . . . . . . . . . . . . 16

2.4.1 Two tone derivation . . . . . . . . . . . . . . 16

2.4.2 Small signal approximation . . . . . . . . . . . . . 22

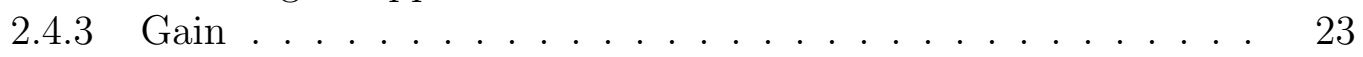

2.4.4 RF noise figure . . . . . . . . . . . . . . . . . . . 24

2.4 .5 Distortion . . . . . . . . . . . . . . . 25

2.4.6 Spurious free dynamic range . . . . . . . . . . . . . . . 25

2.5 Mach-Zehnder interferometer . . . . . . . . . . . . 26

2.6 Complementary linear-field demodulation . . . . . . . . . . . . 27

2.6.1 Noise and gain . . . . . . . . . . . . . . . . . 30

2.6.2 Transfer function curvature . . . . . . . . . . . . . . 31

2.6.3 Residual intensity modulation . . . . . . . . . . . . . . 35

2.6.4 Dispersion . . . . . . . . . . . . . . . . . . . 38

2.7 Summary . . . . . . . . . . . . . . . . . . . . 40

3 Simulated filter performance $\quad 41$

3.1 Filter coefficients . . . . . . . . . . . . . . . . . . 41

3.2 Scaling with filter order . . . . . . . . . . . . . . . . . . . . . . . . . . . . . 44

3.3 Numerical link simulation . . . . . . . . . . . . . . . . . . . 47

3.4 Summary . . . . . . . . . . . . . . . . . . 49 
4 Phase modulation experiments $\quad 52$

4.1 Planar lightwave circuit filters . . . . . . . . . . . . . . . . . . 52

4.2 Implementation and characterization . . . . . . . . . . . . 54

4.3 Link Results . . . . . . . . . . . . . . . . . . . . 55

4.3.1 Phase-modulated link with FIR filter . . . . . . . . . . . 55

4.3.2 Phase-modulated link with IIR filter . . . . . . . . . . . 61

4.4 Summary . . . . . . . . . . . . . . . . 63

5 Frequency modulation experiments $\quad 65$

5.1 Review of FM lasers . . . . . . . . . . . . . . . . 65

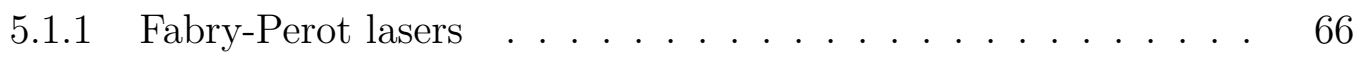

5.1 .2 DBR lasers . . . . . . . . . . . . . . . . 66

5.1.3 DFB lasers . . . . . . . . . . . . . . . . 67

5.2 Laser characterization . . . . . . . . . . . . . . . . 68

5.3 Frequency-modulated link with IIR filter . . . . . . . . . . . . . 72

5.4 Summary . . . . . . . . . . . . . . . . . . . . . 74

6 Conclusions and future work $\quad 75$

$\begin{array}{ll}\text { A Simulation code } & \mathbf{7 7}\end{array}$

A.1 Small-signal simulation f . . . . . . . . . . . . . 77

A.2 Large-signal simulation . . . . . . . . . . . . . . . . 80

A.3 Numerical simulation . . . . . . . . . . . . . . . . . . . . . 81

A.4 Link response . . . . . . . . . . . . . . . . . . . . 83

A.5 Link metrics . . . . . . . . . . . . . . . . . . . 84

$\begin{array}{lr}\text { Bibliography } & 86\end{array}$ 


\section{List of Figures}

1.1 Microwave photonics frequencies of interest. . . . . . . . . .

1.2 Noise and distortion limitations on the dynamic range of a signal transmission system. . . . . . . . . . . . . . . . . .

1.3 Diagram of signal propagation in a microwave photonic link. The output of the link is the original input signal with the addition of noise and distortion. . . . . . . . . . . . . .

1.4 Directly modulated IM-DD link comprised of a semiconductor laser, optical fiber span and photodetector. . . . . . . . . . . . 5

1.5 Externally modulated IM-DD link comprised of a laser, Mach-Zehnder intensity modulator, optical fiber span, and photodetector. . . . . . 5

1.6 Harmonic distortion. . . . . . . . . . . . . . . . . . . . . 6

1.7 Intermodulation distortion. $\ldots \ldots \ldots \ldots \ldots$

1.8 Two tone test. . . . . . . . . . . . . . . . . 6

1.9 Output intercept points and spurious free dynamic range. $\quad \ldots \ldots .7$

1.10 Electrical link dynamic range example with list of typical parameters. 8

1.11 Photonic link dynamic range example with list of typical parameters. $\quad 9$

2.1 Externally modulated PM-DD link comprised of a laser, lithium niobate phase modulator, optical fiber span, optical filters and photodetector. . . . . . . . . . . . . . . . . . . .

2.2 Directly modulated FM-DD link comprised of a multi-section laser, optical fiber span, optical filters and photodetector. . . . . . . . .

2.3 PM-DD link using a Mach Zehnder interferometer, and an IM-DD link with a dual-output Mach Zehnder modulator. For a given photocurrent, these links have the same figures of merit. The IM-DD link may use a multiplexing scheme to combine both complementary signals onto the same optical fiber.

2.4 Ideal filter transfer functions for an optical PM or FM discriminator in a complementary linear-field demodulation scheme. . . . . . . . .

2.5 Phase noise limited noise figure versus linewidth and modulation efficiency, assuming a $50 \mathrm{ohm}$ impedance. . . . . . . . . . .

2.6 Illustration of the quadratic envelope on the transfer function that bounds the second-order figures of merit for the complementary linearfield discriminator. . . . . . . . . . . . . . . . . . . .

6

.

7

8

9


2.7 Illustration of the cubic envelope on the transfer function that bounds the third-order figures of merit for the complementary linear-field discriminator. . . . . . . . . . . . . . . . . .

2.8 Monte Carlo simulation results to test the suitability of the derived bounds on the OIP2 and OIP3. Each point is the worst case of 1000 trials with random errors, and is compared to the analytical bounds. We assume closely spaced tones around $2 \mathrm{GHz}, 1 / 10 \mathrm{GHz}$ slope, 5 $\mathrm{mA}$ of current per detector $\left(i_{d c}=10 \mathrm{~mA}\right), 50 \mathrm{ohm}$ impedance, and 0.5 amplitude bias, $T=0.25$. The analytical expression bounds the simulation within less than $2 \mathrm{~dB} . \ldots \ldots \ldots$

$2.9 \mathrm{OIP}_{3}$ and $\mathrm{SFDR}_{3}$ for an ideal discriminator for different values of residual intensity modulation, assuming closely spaced tones around $2 \mathrm{GHz}, 5 \mathrm{~mA}$ of current per detector $\left(i_{d c}=10 \mathrm{~mA}\right), 50 \mathrm{ohm}$ impedance, and 0.5 amplitude bias, $T=0.25 \ldots \ldots \ldots$

2.10 OIP3 for complementary linear-field discriminators for different slope values and fiber dispersion, assuming standard $\mathrm{SMF}$, with $D=-20 \mathrm{ps}^{2} / \mathrm{km}$, closely spaced tones around $2 \mathrm{GHz}, 5 \mathrm{~mA}$ of current per detector $\left(i_{d c}=\right.$ 10mA), $50 \mathrm{ohm}$ impedance, and 0.5 amplitude bias, $T=0.25 . \quad$.

3.1 Transfer functions for the FIR discriminators optimized at midband. .

3.2 Simulated OIP3 for the three different 10th order FIR filter sets optimized at midband versus normalized modulation frequency. The photocurrent is scaled for $10 \mathrm{~mA}$ total photocurrent (5 mA per detector). The filter is more linear for lower modulation frequencies, and gets worse for large modulation frequencies. For the least squares fit filters, the local minima for certain modulation frequencies are apparent in the plot.

3.3 Simulated OIP2 for the 10th order maximally linear FIR filter set optimized at midband versus common mode rejection ratio. The CMRR is given in decibels of current suppressed. The photocurrent is scaled for $10 \mathrm{~mA}$ total photocurrent (5 mA per detector). The normalized modulation frequency is 0.03 , but no dependence of OIP2 on modulation frequency was observed. For infinite CMRR, the OIP2 value was limited by the numerical precision of the calculation.

3.4 Simulated OIP3 for maximally linear FIR filters, of different order, optimized at midband versus normalized modulation frequency. The photocurrent is scaled for $10 \mathrm{~mA}$ total photocurrent $(5 \mathrm{~mA}$ per detector.$\ldots \ldots \ldots \ldots \ldots \ldots$

3.5 Spurious free dynamic range versus filter order for $5 \mathrm{GHz}$ PM-DD links using maximally linear filters and $200 \mathrm{GHz}$ FSR. The link parameters are given in Table 3.3 on page 47.

3.6 Spurious free dynamic range for $5 \mathrm{GHz}$ PM-DD links using maximally linear filters for various FSR.

3.7 Numerical model of a PM-DD or FM-DD photonic link with two discriminator filters and balanced detection . . . . . . . . . . . . 
3.8 Link response versus input power for a $5 \mathrm{GHz}$ PM-DD link using tenthorder maximally linear filters. The link parameters are given in the text. . . . . . . . . . . . . . . . . .

3.9 Link response versus input power for a $5 \mathrm{GHz}$ PM-DD link using maximally linear filters of different order.

3.10 Spurious free dynamic range versus bandwidth for $5 \mathrm{GHz}$ PM-DD links using maximally linear filters of different orders.

4.1 FIR lattice filter architecture . . . . . . . . . . . . . . . 53

4.2 Tunable PLC FIR lattice filter architecture . . . . . . . . . . . 53

4.3 (a) Filter stage for an FIR lattice filter (b) Filter stage for an IIR, RAMZI filter. . . . . . . . . . . . . . 55

4.4 Photograph of single FIR filter with wiring board inside protective box. 56

4.5 Photograph of single FIR filter mounted on heat sink. . . . . . . . . 56

4.6 Diagram of the system used for characterization . . . . . . . . . . 57

4.7 Photograph of current amplifier board to drive the chrome heaters on the tunable filters. . . . . . . . . . . . . . . . 57

4.8 Photograph of National Instruments analog input/output card interface. . . . . . . . . . . . . . . . 58

4.9 Achieved filter amplitude and phase for the 6th order FIR lattice filter. 59

4.10 Fundamental and third-order intermodulation distortion versus laser wavelength. The modulation power is fixed at $10 \mathrm{dBm}$ and the photocurrent is fixed at $0.11 \mathrm{~mA}$. . . . . . . . . . . . . . .

4.11 Fundamental and third-order intermodulation distortion versus modulation power. The photocurrent is fixed at $0.11 \mathrm{~mA}$ and the wavelength is fixed at $1593.7 \mathrm{~nm}$.

4.12 Achieved filter amplitude and phase for the RAMZI filter.

4.13 Output intercept point of third-order intermodulation distortion versus laser wavelength in simulation and experiment. The total photocurrent is fixed at $10.5 \mathrm{~mA}$ and the modulation frequency is $5 \mathrm{GHz}$. The theoretical OIP3 of a link with a dual-output MZM and the same received photocurrent is also plotted in the figure.

4.14 OIP3 and OIP2 versus modulation frequency at a fixed photocurrent of $10.5 \mathrm{~mA}$ and wavelength of $1549.964 \mathrm{~nm}$.

4.15 Output power versus modulation power compared to a dual-output Mach-Zehdner modulator measured experimentally. The frequency is fixed at $3.3 \mathrm{GHz}$ and the effective DC photocurrent at $141 \mathrm{~mA}$. . . .

4.16 OIP3 versus effective DC photocurrent. The frequency is fixed at 4.0 $\mathrm{GHz}$ and the modulation power at $0 \mathrm{dBm} . \ldots . . . . . . .664$

5.1 Self heterodyne laser linewidth measurement experimental setup. . . . 68

5.2 Self heterodyne laser spectrum measurements with Lorentzian fits. . . 69

5.3 DC tuning measurement of DBR laser phase sections. . . . . . . . . . 70

5.4 FM modulation efficiency experimental setup. . . . . . . . . . . . 70

5.5 DBR FM modulation efficiency versus frequency. . . . . . . . . . . 71 
5.6 Phase-noise limited noise figure for FM DBR lasers from measured modulation efficiency and linewidth. . . . . . . . . . . . 71

5.7 Residual intensity modulation measurement of DBR FM lasers. . . . 72

5.8 Link gain versus modulation frequency for the FM link versus the PM+IIR link. . . . . . . . . . . . . . . . . . . 73

5.9 Distortion versus modulation frequency, compared to the results of the PM+IIR link. . . . . . . . . . . . . . . 73 


\section{List of Tables}

2.1 Approximations to the noise figure expressions for arbitrarily filtered links. These assume large positive gain with either shot or phase noise limited noise figures. Shot noise limits occur for moderate optical powers and phase noise limit occurs for much larger optical powers. These approximations are not valid if the link attenuates the rf power. . . .

2.2 General expressions for $O I P 2$, OIP3, and spurious free dynamic range for an abitrarily filtered link with either phase or frequency modulation and direct detection given in terms of the link distortion constants. SFDR is limited by either shot or phase noise, and second-order or third-order distortion. . . . . . . . . . . . . . . . . .

2.3 Figures of merit for an PM-DD link with an a-MZI and balanced de-

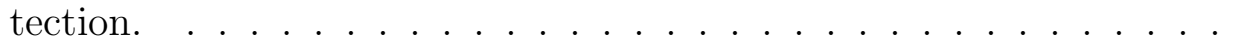

2.4 Gain and noise figure expressions for the complementary linear-field demodulated PM-DD link. . . . . . . . . . . . . . .

2.5 Expressions for the worst case $\mathrm{OIP}^{2}, \mathrm{OIP}^{3}$, and spurious free dynamic range for complimentary linear-field demodulation limited by filter cur-

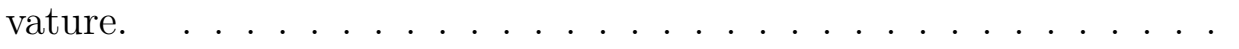

2.6 Expressions for OIP2 and OIP3 for complimentary linear-field demodulation limited by residual intensity modulation, with an arbitrary phase difference between the angle modulation and the intensity modulation. The frequency dependent terms are only a small correction for closely spaced tones. . . . . . . . . . . . . . . . .

3.1 Filter coefficients for negative slope and positive slope, midband optimized, 10th order, FIR discriminators. Each filter is symmetric, so half the coefficients are duplicated. The symmetric filters are guaranteed to have linear phase. The first least squares fit is optimized for normalized frequencies 0.3 to 0.7 , and the second least squares fit is optimized for normalized frequencies 0.45 to 0.55 . The coefficients for the maximally linear filter are from the cited reference. All three filters are Type I linear phase FIR filters (odd-length, symmetric). . . . . . 
3.2 Filter coefficients for the 2nd, 6th, 10th, 14th, and 18th order maximally linear filters in z-transform representation. Each filter is symmetric, so half the coefficients are duplicated. The coefficients given are for the positive slope filters. For negative slope filters, the even-numbered coefficients have opposite sign. . . . . . . . . . . . . . 45

3.3 Simulation parameters . . . . . . . . . . . . . . . . . 47

4.1 Filter phase and coupler parameters for a tenth-order maximally linear discriminator filter in lattice filter form . . . . . . . . . . . . . 


\section{Acknowledgments}

This work would not have been possible if it were not for the help of a great many people. First, I would like to thank my parents for instilling an appreciation for education, and for their love and support as my graduate studies brought me away to California. My advisor, Professor Ming C. Wu, has provided advice, resources and patience during the completion of my dissertation.

A special thanks goes to my research collaborators at Harris Corporation, especially Charles Middleton, Scott Meredith, Robert Peach, and Richard DeSalvo, and those at Alcatel-Lucent Bell Laboratories, including Mahmoud Rasras, Liming Zhang, and Y. K. Chen. Funding and guidance from the Defense Advanced Research Projects Agency (DARPA) has been instrumental in the completion of this work. I would like to thank Prof. Connie Chang-Hasnain, Prof. Xiang Zhang, and Prof. Paul Wright for serving on my exam or dissertation committees.

Finally, my academic colleagues at Berkeley have been indispensable for their stimulating discussions, contributions, and friendships, especially Erwin Lau, Devang Parekh, Alex Grine, Niels Quack, Amit Lakhani, Tae Joon Seok, Jeff Chou, ByungWook Yoo, and Trevor Chan. 


\section{Chapter 1}

\section{Introduction}

The impact of photonics on digital communication systems is extensive and well known. Fiber optics carry massive amounts of data between users and services around the globe. These systems are finding applications in shorter and shorter distances, from long-distance telecommunications, to communication between servers in data centers, to interconnects within computers themselves. The large bandwidths of photonic systems are enabling this revolution.

Less well known are the benefits of photonics to high-frequency analog systems. These "microwave photonic" systems are analogous to radio systems, where baseband signals are modulated onto a carrier frequency. Photonics provide very high frequency carriers, around $194 \mathrm{THz}$ for $1550 \mathrm{~nm}$ wavelength light used with standard single mode fiber, so signals being transmitted and manipulated are relatively low frequency in comparison. RADAR and wireless communications are two areas that can greatly benefit from microwave photonics.

Improvements in the analog performance of photonic systems, especially reductions in noise and distortion, have direct application back to digital communications. Next generation, commercial, digital fiber-optic communication systems are improving spectral efficiency (bits/s/Hz) over existing fibers in order to save on infrastructure upgrades to fiber optic networks. They are moving away from simple on-off-key (OOK) representations of digital data in favor of multi-level and coherent modulation techniques. Optimizing the analog performance of photonic devices and systems increases the achievable spectral efficiencies in these coherent systems, and empowers this next advance in communications.

\subsection{Microwave photonics applications}

Microwave photonics is the study of photonic devices, such as lasers and photodetectors, performing operations at microwave frequencies, and the application of these devices to microwave systems. Microwave photonics has been extensively reviewed by [1-4], and tutorial information has been published in book form by $[5,6]$. The field broadly defines the word "microwave" to include frequencies ranging from hundreds of megahertz to a terahertz. Much work has been performed in the Super High Fre- 
Figure 1.1: Microwave photonics frequencies of interest.

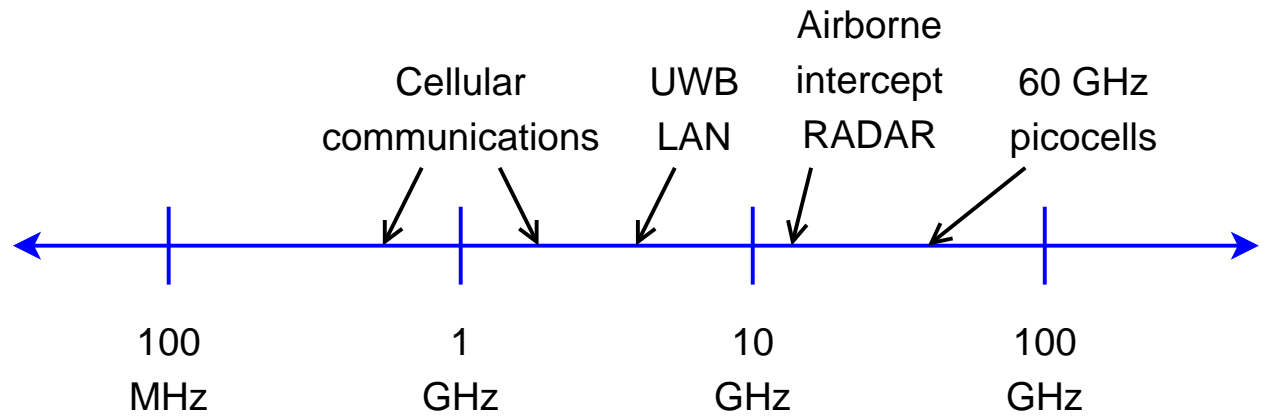

quency (SHF) band, defined by the International Telecommunication Union (ITU), which ranges from $3 \mathrm{GHz}$ to $30 \mathrm{GHz}$. A variety of RADAR and wireless communication frequencies fall within this band. Microwave photonic systems are analog systems. They are analog in the sense that they manipulate arbitrary baseband signals as well as digital signals that are modulated onto a higher carrier frequency.

The main applications for microwave photonics can be categorized into signal transmission and signal processing. Photonics can be used for antenna remoting and signal distribution for a variety of radio technologies. For example, an array of CDMA antennas are used to extend cellular coverage to the interior of a large building such as a railway station, airport or subway. Each individual antenna transmits the detected signals via microwave photonic links back to a single central location for processing. With the right design, the power consumption at each of the nodes can be made very small, and each node can be small and inexpensive [2].

In another example, [7], an array of radar antennas on a large military aircraft are connected to a central location with microwave photonic links. The array concept improves the overall sensitivity of the system over discrete transmitters, and photonics allows low-loss collection of the signals.

Signal processing can also be performed with microwave photonics. Researchers have implemented diverse functions such as tunable bandpass and notch filtering of interference [8], microwave mixing [9], arbitrary waveform generation [10], and wide band analog to digital conversion [11]. Photonics can be used for the generation of microwave signals. Optoelectronic oscillators (OEOs) are one technique which can produce very low-noise microwave oscillation [12]. Photonics can also generate millimeter wave signals through frequency multiplication techniques, such as with with injection locked lasers. The wide bandwidth of microwave photonics makes it ideal for performing these signal processing functions. 


\subsection{Advantages for signal distribution}

For signal distribution, the competition to photonics is coaxial cabling. Conventional systems are fed electronically with coaxial cable from the processing station. Electronic feeds (which are 3-300 meters in shipboard and avionics) have low efficiencies in size, weight and power (SWAP). These feeds are relatively large, inflexible and heavy because of multiple coax cable runs. They have high loss, which limits the range and requires amplification at the antennas. Coax is not especially wide bandwidth because its attenuation is frequency dependent. Coax is also susceptible to electromagnetic interference (EMI), which is undesirable in military applications.

Microwave photonic links have been explored for replacing traditional coaxial links in a variety of applications because of their many advantages [13-15]. Optical fibers have significant advantages in size and weight over microwave coax. Fiber has a thin cross section and its bend radius is much tigher than for coax. By remoting signals with fiber, the power burden can be shifted to the processing station. Fibers are low loss, and the loss does not depend very much on the signal frequency. Several signals can be multiplexed on the same fiber using wavelength division multiplexing. Fiber is immune from EMI.

The most successful commercial applications have been in hybrid-fiber-coax (HFC) infrastructure for distributing cable-television signals and in hybrid-fiber-radio (HFR) for distributing cellular signals to remote antennas [6,13]. Military radar and communication systems use analog fiber optic systems for antenna remoting. However, advanced military and next generation wireless systems need a large dynamic range of operation. This is challenging for microwave photonics, as they are not yet competitive with electronic systems in terms of noise and distortion [16]. In addition, large dynamic range is important for microwave photonics signal processing, and microwave photonic links are a performance limiting component of these systems. By improving the performance of the microwave photonic links, the full systems also are improved. The research question addressed in this work is whether we can have the advantages of fiber for microwave signal transmission while still maintaining a large dynamic range.

\subsection{Dynamic range challenges}

The dynamic range is the range of signal amplitudes that can be transmitted or processed by a system. In the wireless antenna remoting example, the dynamic range will play a role in determining the size and capacity of each cell. Remote-units located

close to the antenna have to limit their power and transmission rate if they exceed the upper end of the dynamic range, and remote units located far from the antenna will not be noticeable if they fall below the lower end of the dynamic range. At the lower end, the range is limited by noise, and at the upper end, often limited by the point where distortion of the signal by the system is noticeable. Distortion produces harmonics and mixtures between signal frequencies, and at a high enough signal power, these products become larger than the noise. This particular definition of dynamic 
Figure 1.2: Noise and distortion limitations on the dynamic range of a signal transmission system.
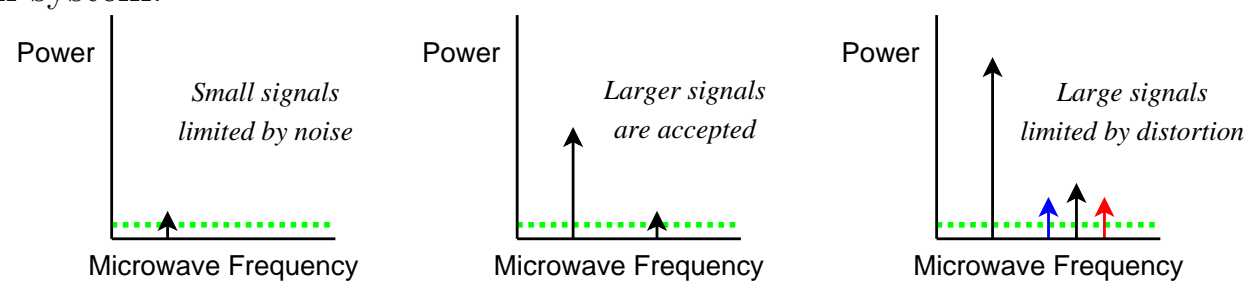

range is called the spurious free dynamic range. The largest distortion products tend to be the second and third orders, which grow quadratically and cubically with the input power. Fig. 1.2 illustrates the concept of dynamic range.

In the following sections, I will define relevant concepts, and then will give an example comparison between an electrical link and a microwave photonic link, which shows the limitations of the photonic system in terms of dynamic range.

\subsubsection{Microwave photonic links}

A microwave photonic link modulates arbitrary analog signals on a high frequency carrier. For $1550 \mathrm{~nm}$ light, the carrier is approximately $194 \mathrm{THz}$. The analog signals can be divided into frequency bands, for example, 0.1-4 GHz, 4-8 GHz, and 8-12 GHz. In each, an RF carrier has baseband data modulated upon it. The modulation process creates optical sidebands on the optical carrier. It also adds noise due to the phase and intensity noise of the laser, and distorts the signal. The detection process recovers the electrical signal, but also adds additional noise and distortion due to shot noise and nonlinearities in the photodetection. Fig. 1.4 illustrates the steps in a microwave photonic link.

Typical microwave photonic links uses intensity modulation and direct detection (IM-DD). These links will be the baseline for comparison in later sections. Fig. 1.4 illustrates a direct modulated IM-DD link, where the bias current to the laser is varied with the signal, thus varying the intensity of the emitted light. Fig. 1.5 illustrates an externally modulated IM-DD link, where a lithium-niobate Mach-Zehnder modulator is used to attenuate the laser light in proportion to the signal.

\subsubsection{Distortion}

Distortion includes both harmonic distortion and intermodulation distortion. Harmonic distortion creates multiples of a modulation frequency. It is typically out-ofband, but this is still important for multiband links and ultra-wideband links. Intermodulation distortion (IMD) or "intermod" is when signals of different frequencies are mixed. Typically, the most important IMD terms are 3rd order sum-and-difference products, which fall in-band. For example, for two modulation frequencies $f_{1}$ and $f_{2}$, the important mixing terms are $2 f_{2}-f_{1}$ and $2 f_{1}-f_{2}$.

Distortion is typically quantified using a two-tone-test. Two closely spaced fre- 
Figure 1.3: Diagram of signal propagation in a microwave photonic link. The output of the link is the original input signal with the addition of noise and distortion.
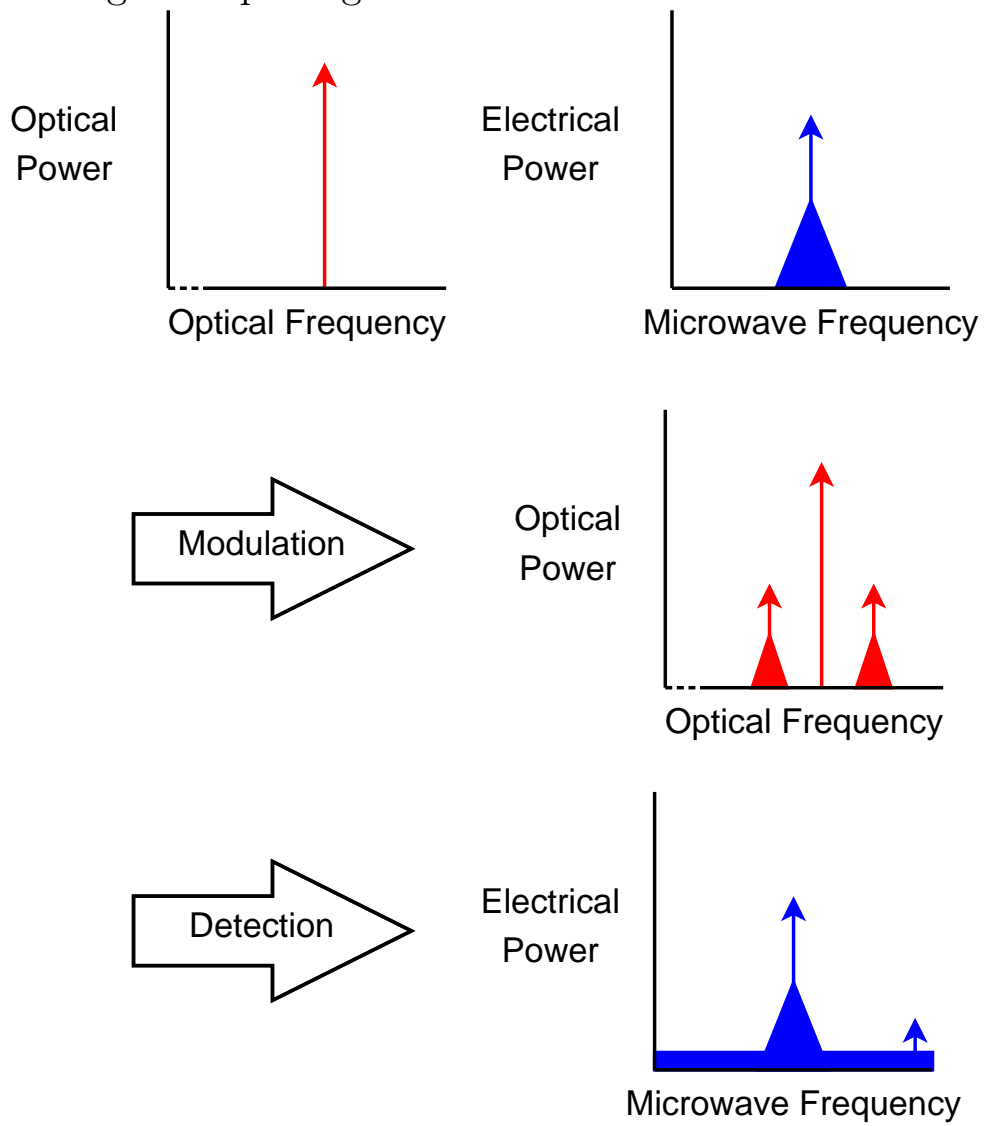

Figure 1.4: Directly modulated IM-DD link comprised of a semiconductor laser, optical fiber span and photodetector.

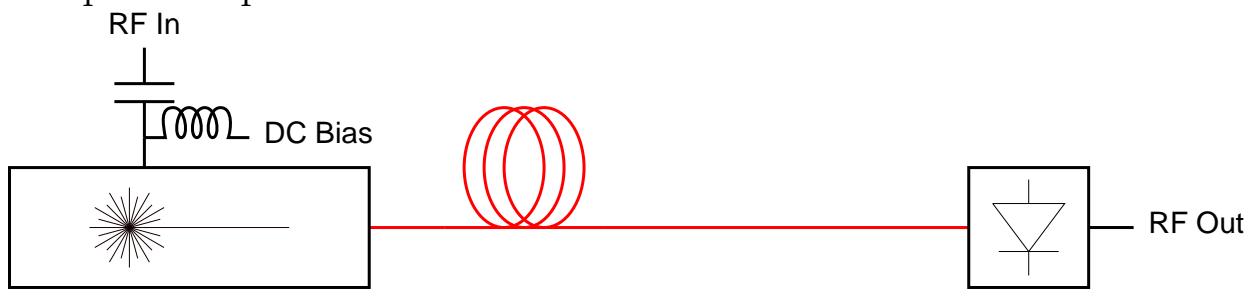

Figure 1.5: Externally modulated IM-DD link comprised of a laser, Mach-Zehnder intensity modulator, optical fiber span, and photodetector.

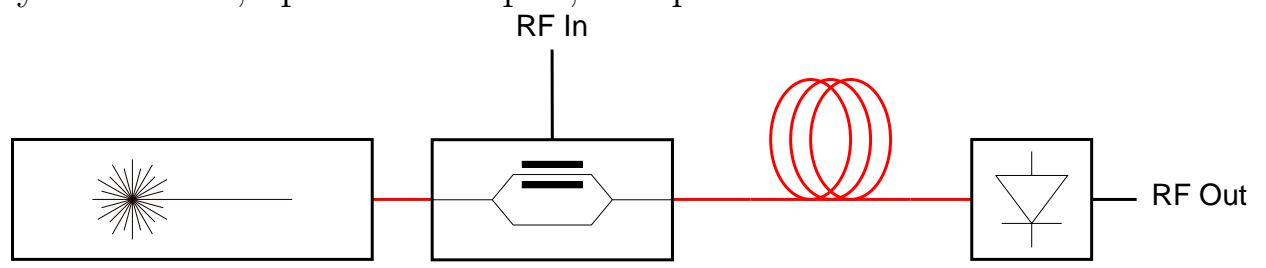




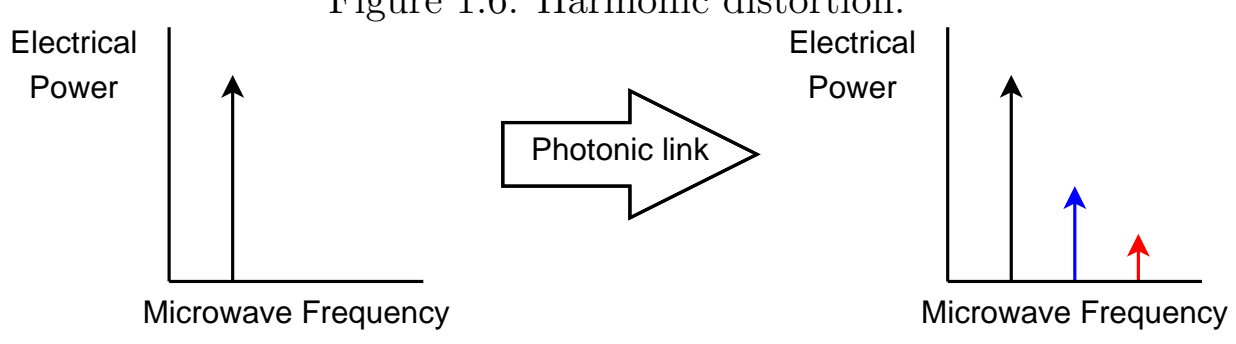

Figure 1.6: Harmonic distortion.
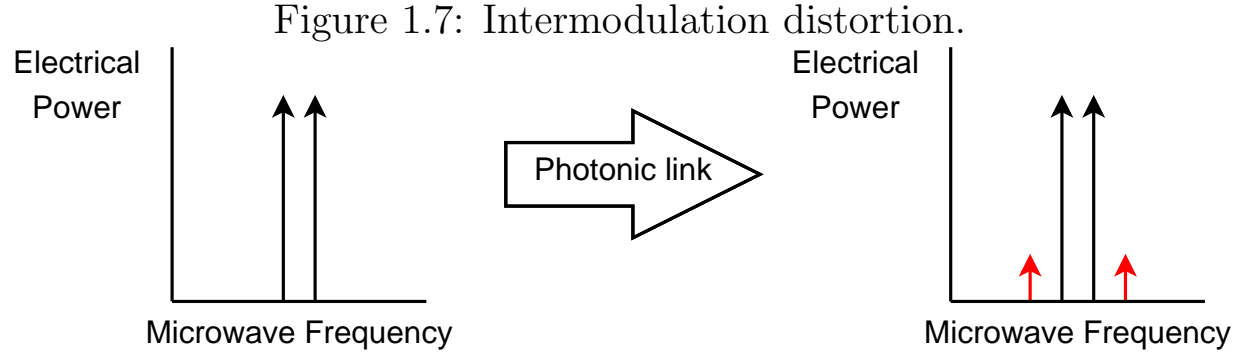

quencies are transmited, and the power in the resulting distortion terms are measured with a spectrum analyzer. Interpolating small signal measurements to high input powers, the points where the distortion terms are equal to the fundamental in power are called the intercept points. The output powers where the second-order distortion and third-order distortion are expected to be equal to the fundamental are the secondorder output intercept point (OIP2) and third-order output intercept point (OIP3). Larger values for OIP2 and OIP3 mean less distortion.

\subsubsection{Noise}

Laser relative intensity noise (RIN), laser frequency and phase noise, optical shot noise and modulator/detector thermal noise all contribute to the noise of the link. The noise of the link is quantified by its noise figure. The noise figure is given by the input's signal to noise ratio divided by the output's signal to noise ratio, usually assuming the input is thermal noise limited in a $50 \mathrm{ohm}$ impedance. A smaller noise figure link introduces less noise. The noise of the link combined with the distortion is also quantified by the spurious free dynamic range (SFDR) of the link.

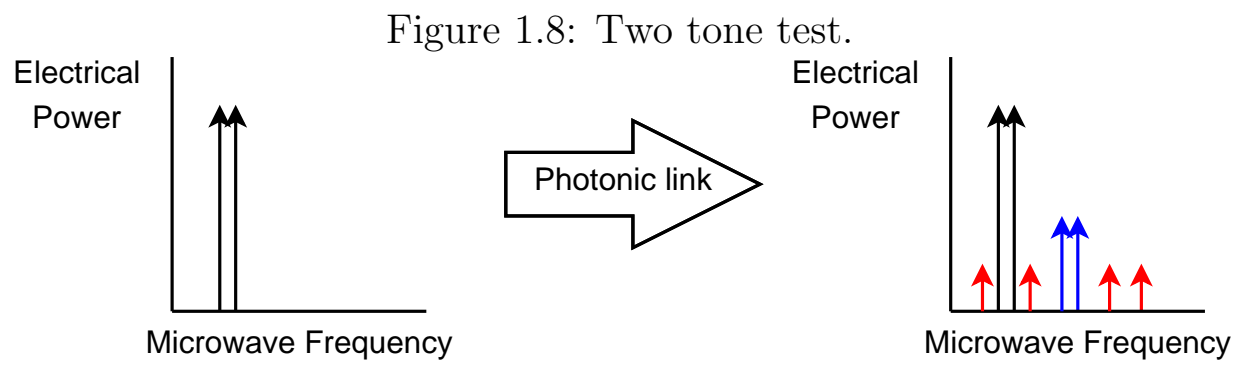


Figure 1.9: Output intercept points and spurious free dynamic range.

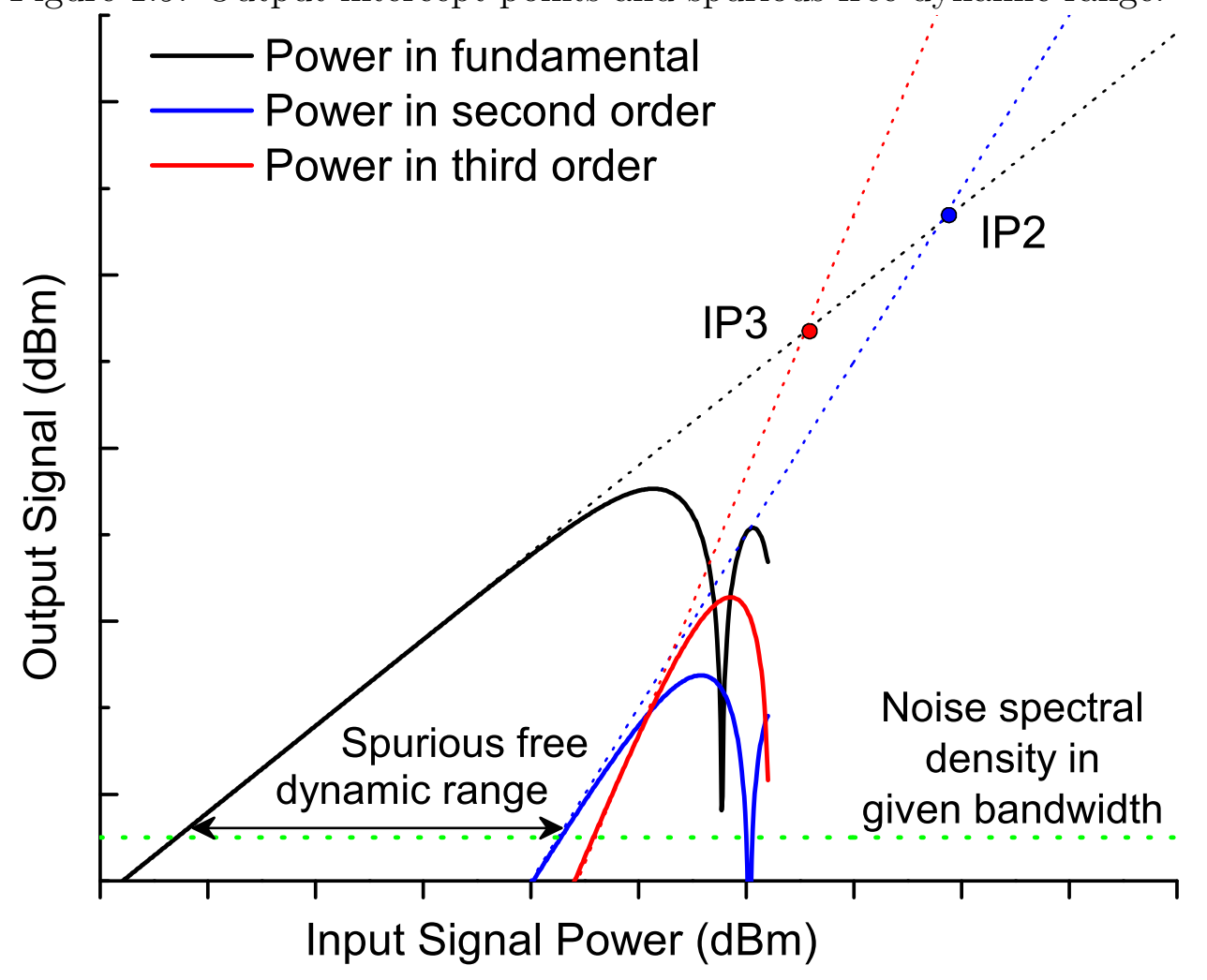

\subsubsection{System example}

I would like to give an example that illustrates the dynamic range of a very good electronic link compared to a microwave photonic link. Suppose I have to transmit a signal centered at $2 \mathrm{GHz}$ frequency over a distance of $100 \mathrm{~m}$. Very low attenuation, high performance coaxial cabling has been developed for avionics. At best, these cables have an attenuation of $20 \mathrm{~dB}$ per $100 \mathrm{~m}$. Typical commercial cabling has much higher attenuation.

Assume I place a high-dynamic-range pre-amplifier before the link to overcome the $20 \mathrm{~dB}$ attenuation. I assume a gain of $20 \mathrm{~dB}$, a $1 \mathrm{~dB}$ noise figure, and a thirdorder output intercept point of $10 \mathrm{~W}(40 \mathrm{dBm})$. Amplifiers are typically limited by third-order distortion, so the OIP3 value is relevant to calculating the spurious free dynamic range. In decibel units, the SFDR is given by

$$
S F D R=\frac{2}{3}\left(O I P 3-G+174 \frac{\mathrm{dBm}}{\mathrm{Hz}}-10 \log _{10}(B)-N F\right)
$$

where $G$ is the gain in $\mathrm{dB}$ units and $B$ is the bandwidth. In $1 \mathrm{~Hz}$ bandwidth, this would give a dynamic range of $129 \mathrm{~dB}$. (75 dB in $100 \mathrm{MHz}$ of bandwidth). The link noise figure is limited to the noise figure of the amplifier, and is about $1 \mathrm{~dB}$.

I will next illustrate the dynamic-range of a typical photonic link using commercially available components. This system consists of an electrical to optical (e-to-o) transducer, a fiber optic transmission line, and an optical to electrical (o-to-e) trans- 
Figure 1.10: Electrical link dynamic range example with list of typical parameters.

\begin{tabular}{|l|l|}
\hline Parameter & Value \\
\hline \hline Signal frequency & $2 \mathrm{GHz}$ \\
\hline Distance & $100 \mathrm{~m}$ \\
\hline Coaxial cable & Low loss PTFE dielectric or 0.325 in rigid coax \\
\hline Attenuation & $20 \mathrm{~dB} / 100 \mathrm{~m}$ \\
\hline Amplifier gain & $20 \mathrm{~dB}$ \\
\hline Amplifier noise figure & $1 \mathrm{~dB}$ \\
\hline Amplifier OIP3 & $10 \mathrm{~W}(40 \mathrm{dBm})$ \\
\hline Spurious free dynamic range & $129 \mathrm{~dB}$ in $1 \mathrm{~Hz}$ bandwidth \\
\hline Noise figure & $1 \mathrm{~dB}$ \\
\hline
\end{tabular}

ducer. Our e-to-o transducer is a high efficiency Mach-Zehnder modulator, which modulates a microwave signal onto the intensity of an optical carrier provided by a semiconductor laser. The o-to-e transducer is a photodiode, which detects the envelope of the intensity modulation. For $100 \mathrm{~m}$ of single-mode optical fiber, the transmission loss is less than $0.05 \mathrm{~dB}$, which is why fiber optics are extensively used for long distance communications. The parameters below were chosen to give a gain of $0 \mathrm{~dB}$ for the link.

The e-to-o transducer has a sinusoidal transfer function of light intensity versus voltage, which contributes a large amount of distortion to the final signal. This system requires a photodiode capable of handling high optical power. Research devices have been demonstrated that can handle much higher powers than this, but this is still an expensive device. The third-order distortion and shot noise limited SFDR for this link is derived in $\mathrm{dB}$ units per $1 \mathrm{~Hz}$ bandwidth by [17] as

$$
S F D R=\frac{2}{3} \cdot 10 \log _{10}\left(\frac{2 I_{d c}}{e}\right)
$$

where $e$ is the elementary charge and $I_{d c}$ the effective DC photocurrent. In $1 \mathrm{~Hz}$ of bandwidth, this would give a dynamic range of $116 \mathrm{~dB}$, which is $13 \mathrm{~dB}$ worse than the electronics case. What's worse is the noise figure of this particular link, which is $18.5 \mathrm{~dB}$, compared to $1 \mathrm{~dB}$ for the electronics case. Assuming a shot-noise limited receiver, the noise figure is calculated by using [17]

$$
N F=10 \log _{10}\left(\frac{2 e V_{\pi}^{2}}{I_{d c} \pi^{2} K T Z_{\text {in }}}\right)
$$


Figure 1.11: Photonic link dynamic range example with list of typical parameters.

RF In

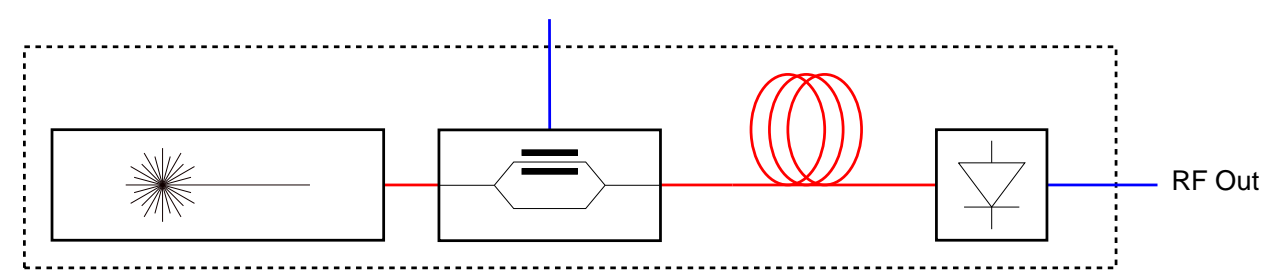

\begin{tabular}{|l|l|}
\hline Parameter & Value \\
\hline \hline Signal frequency & $2 \mathrm{GHz}$ \\
\hline Distance & $100 \mathrm{~m}$ \\
\hline Fiber attenuation & $<0.05 \mathrm{~dB}$ \\
\hline Modulator & High efficiency Lithium Niobate MZM \\
\hline Halfwave voltage & $3 \mathrm{~V}$ \\
\hline Photodetector & High power InGaAs PIN photodiode \\
\hline Photocurrent & $20 \mathrm{~mA}$ \\
\hline Spurious free dynamic range & $116 \mathrm{~dB}$ in $1 \mathrm{~Hz}$ bandwidth \\
\hline Noise figure & $18.5 \mathrm{~dB}$ \\
\hline
\end{tabular}

where $V_{\pi}$ is the modulator half-wave voltage, $K$ is Boltzmann's constant, $T$ is the system temperature $(300 \mathrm{~K}), Z_{\text {in }}$ is the input impedance of the system, typically 50 ohms.

The noise figure is heavily influenced by the inefficiency of the e-to-o transducer, given by large $V_{\pi}$. In addition, in a real system, the input and output of the system must be impedance matched. If passive impedance matching is used, the usable signal level is further reduced. For better noise and dynamic range performance, I would like to have higher efficiency e-to-o conversion, and e-to-o conversion that is much more linear.

\subsection{Techniques to improve dynamic range}

There has been much work performed to improve the dynamic range of microwave photonic links through both optical design and by using electrical system techniques. The noise and linearity performance of externally modulated photonic links scale with increasing optical power at the detector, as can be seen in equations 1.1 and 1.2. Work has been dedicated to improving the power handling of photodetectors and their linearity $[18,19]$, designing high power handling optical fibers to reduce optical power induced stimulated Brillouin scattering, and reducing laser relative intensity noise to ensure that the receiver is shot noise limited at higher optical powers. On the modulator side, there have been efforts to decrease the halfwave voltages of MachZehnder modulators to improve the link gain. 
Researchers have developed modulator designs which improve the link linearity over that of a simple MZM. These modulators, with multiple modulation sections, have a transfer function that is more linear than the MZM's sinusoidal one [20]. However, linearized modulators are complicated, difficult to fabricate, difficult to optimize for high-frequency (traveling-wave) operation, and have had little experimental demonstration.

Laser designers have worked on improving the direct intensity modulation linearity of semiconductor lasers. There has been interest in modeling and choosing physical device parameters which minimize the distortion (for example, [21]). Strong optical injection locking is one technique which has been shown improve to linearity by increasing laser resonance frequency [22]. System design techniques, including using a push-pull configuration with balanced detection have shown some success [15].

There are electronic means for improving link distortion by compensating for modulation nonlinearity. These include predistortion [23, 24], feedforward linearization techniques [25], and feedback linearization [26]. However, these techniques require fast electronics to perform the linearization. At the present time, they are not useable for very high frequency microwave photonics beyond a few $\mathrm{GHz}$.

In this work, I have demonstrated linearity improvement using two techniques called phase modulation direct-detection (PM-DD) and frequency modulation directdetection (FM-DD). These approaches are based on optical system design and do not require high-speed electronics for linearization, so they are potentially useable to very high modulation frequencies. The modulation techniques are simple, requiring only a lithium niobate phase modulator or a direct-modulated multi-section laser. The demodulation process does require optical filters, but these are realizeable with a variety of fabrication technologies. PM-DD and FM-DD systems scale in performance with detector power handling as do IM-DD links, so they benefit from more general device research in the field. The following chapters will present theoretical derivations, simulations and experimental evidence of the benefits which PM-DD and FM-DD microwave photonic links can provide to improve the noise and linearity in microwave photonic systems. 


\section{Chapter 2}

\section{Theory of PM-DD and FM-DD links}

\subsection{Motivation for phase and frequency modulation}

Microwave photonic links (MPLs) with large dynamic range are an essential component of high-performance microwave distribution and processing systems. Large dynamic ranges require low signal distortion and low noise figures. These metrics are poor in traditional intensity modulated links, but modulation is not limited to the intensity. Other parameters of the light can be used to convey information, including the amplitude, phase, frequency, spatial modes, and polarization of the light's electric field. Phase modulation (PM) and frequency modulation (FM), where the instaneous optical phase or frequency is varied in proportion to the input signal, are considered to be promising alternatives to IM. PM is a promising modulation technique for MPLs because devices are highly linear. Phase modulators based on the linear electro-optic effect, including those fabricated in lithium niobate, are intrinsically linear, and authors have also reported linear, integrable phase modulators fabricated in indium-phosphide [27].

The signal loss of MPLs is an important factor for links and systems as it impacts the signal to noise ratio. Traditional intensity-modulated direct-detection (IMDD) links experience large signal-loss and resulting low noise figures due to the low modulation efficiency of lithium niobate Mach Zehnder modulators (MZMs). On the other hand, directly modulated frequency modulated (FM) lasers have been demonstrated with high modulation efficiency and with modulation bandwidths that are not limited by the laser relaxation frequency [28]. Recent work on multi-section DFB [29] and EML lasers [30] have produced modulation efficiencies two orders of magnitude better than traditional intensity modulation. An improvement in modulation efficiency could make a major impact on the noise performance of microwave photonic links. Besides high modulation efficiency, the performance of these devices is also more linear than direct intensity modulation and Mach Zehnder modulators, and there is low thermal 
Figure 2.1: Externally modulated PM-DD link comprised of a laser, lithium niobate phase modulator, optical fiber span, optical filters and photodetector.

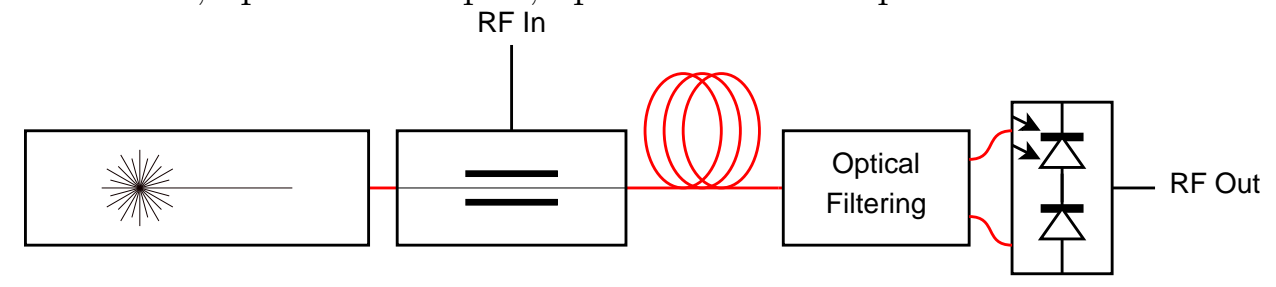

Figure 2.2: Directly modulated FM-DD link comprised of a multi-section laser, optical fiber span, optical filters and photodetector.

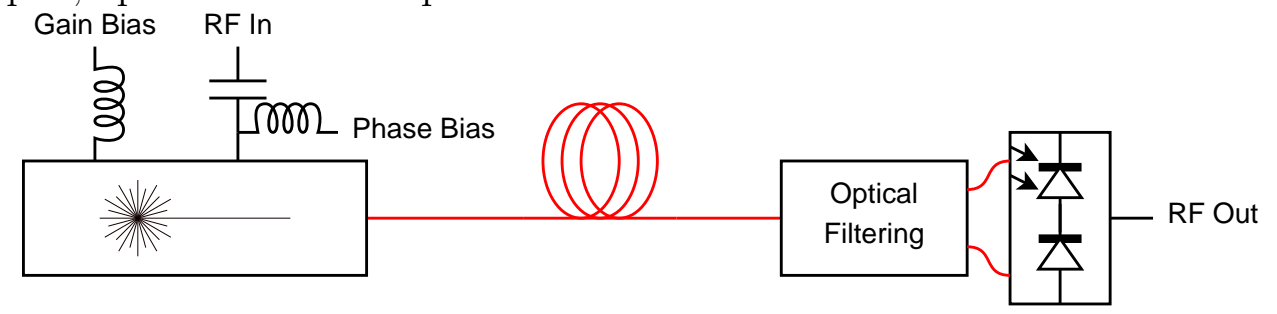

cross-talk in integrated laser arrays. PM and FM have favorable characteristics for linearity and gain in MPLs.

\subsection{Link architecture}

Because photodiodes only respond to the intensity envelope of the light, phase and frequency modulation can not be directly detected. Coherent detection using heterodyning is one possibile demodulation scheme, but heterodyning is nonlinear and adds complexity. Alternatively, one can use a direct-detection system. We have designed demodulators which use optical filters to convert the phase and frequency modulation into AM before direct detection at a photodetector. The filters are called phase and frequency discriminators. The demodulation process is called phase-modulation or frequency-modulation direct-detection (PM-DD or FM-DD [31]), filter-slope detection, or interferometric detection [17]. The architecture for the PM-DD and FM-DD links consists of a modulation source, discriminator filters, and balanced detectors. The link architectures are shown in Fig. 2.1 and Fig. 2.2. Discriminators for PMDD and FM-DD links have similar design because PM is identical to FM but with a modulation depth that is linearly dependent on modulation-frequency.

The sidebands of a phase-modulated or frequency-modulated signal possess certain amplitude and phase relationships among themselves such that the envelope of the signal is independent of time. A discriminator works by modifying these phase and amplitude relationships such that the amplitude of the envelope of the resultant signal fluctuates in the same manner versus time as did the instantaneous frequency of the original signal [32]. One can also think of the discriminator as a filtering function with a frequency dependent amplitude. The slope of the function converts variations in the optical frequency into variations in the amplitude. This view is accurate for slow 
Figure 2.3: PM-DD link using a Mach Zehnder interferometer, and an IM-DD link with a dual-output Mach Zehnder modulator. For a given photocurrent, these links have the same figures of merit. The IM-DD link may use a multiplexing scheme to combine both complementary signals onto the same optical fiber.
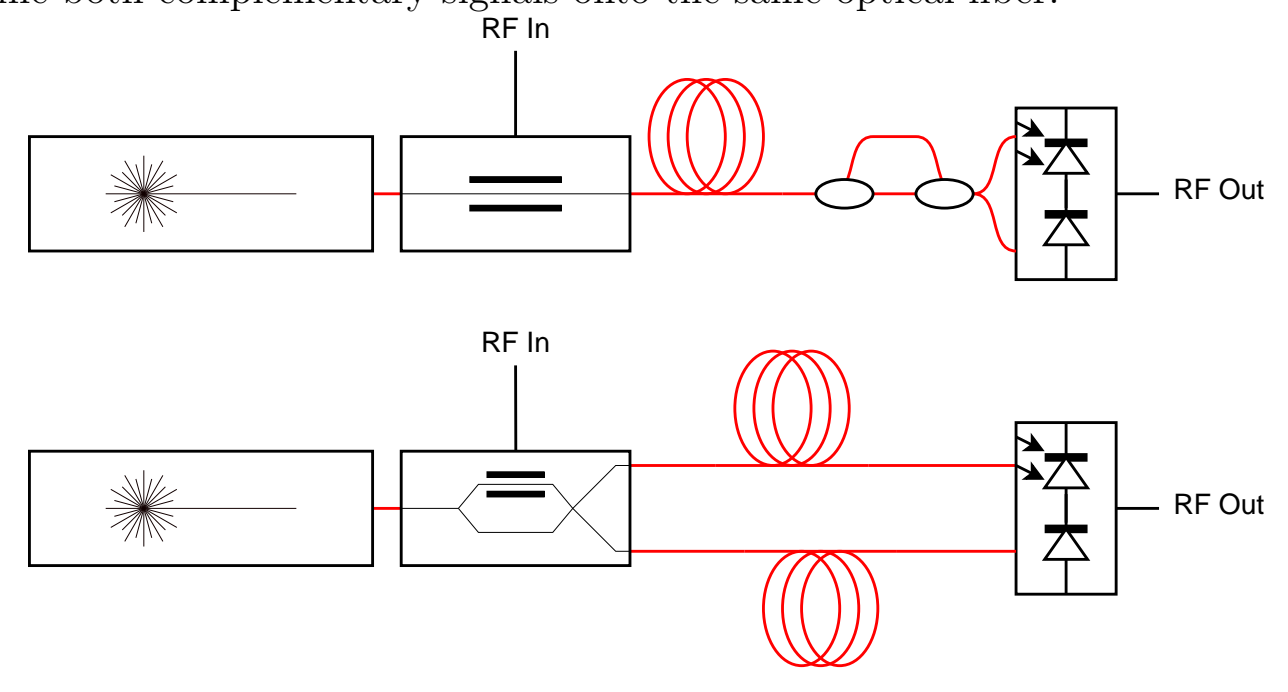

variations of the optical frequency. However, it generally can be misleading since it assumes that the instantaneous frequency of the light is equivalent to a time-averaged frequency. Nevertheless, the model is instructive as it suggests that functions with larger slopes will have higher conversion efficiency to AM, and that a function with many large high order derivatives will distort the AM signal more than one with a more "linear" function.

The system's performance is determined by the transfer function of the optical filter. For example, a Mach Zehnder interferometer (MZI) after a phase modulator has comparable nonlinearity to a Mach Zehnder modulator [17]. This is shown in Fig. 2.3. Authors have proposed various discriminator-filters to optimize the demodulation for low distortion, including birefringent crystals [33], asymmetrical Mach Zehnder interferometers (a-MZI) [17, 34], Fabry-Perot filters [35], fiber Bragg gratings [36] and tunable integrated filters [37, 38].

In the PM-DD and FM-DD links, the ideal transfer function of the optical filter is a linear ramp of field-transmission versus offset frequency from the optical carrier, which is a quadratic ramp of power transmission. The ideal filters have linear phase. The power is split between two filters with complementary slope, and detected with a balanced photodetector. I first analyzed this complementary linear-field demodulation scheme analytically in [39]. The link architecture is shown in Fig. 2.4. A single filter and detector has low third-order distortion, and the balanced detection cancels second-harmonics of the signal's Fourier-frequency components produced by squaring of the AM. Since it is difficult to implement this transfer function in optics, a realized discriminator will have a transfer function with some non-idealities. 
Figure 2.4: Ideal filter transfer functions for an optical PM or FM discriminator in a complementary linear-field demodulation scheme.

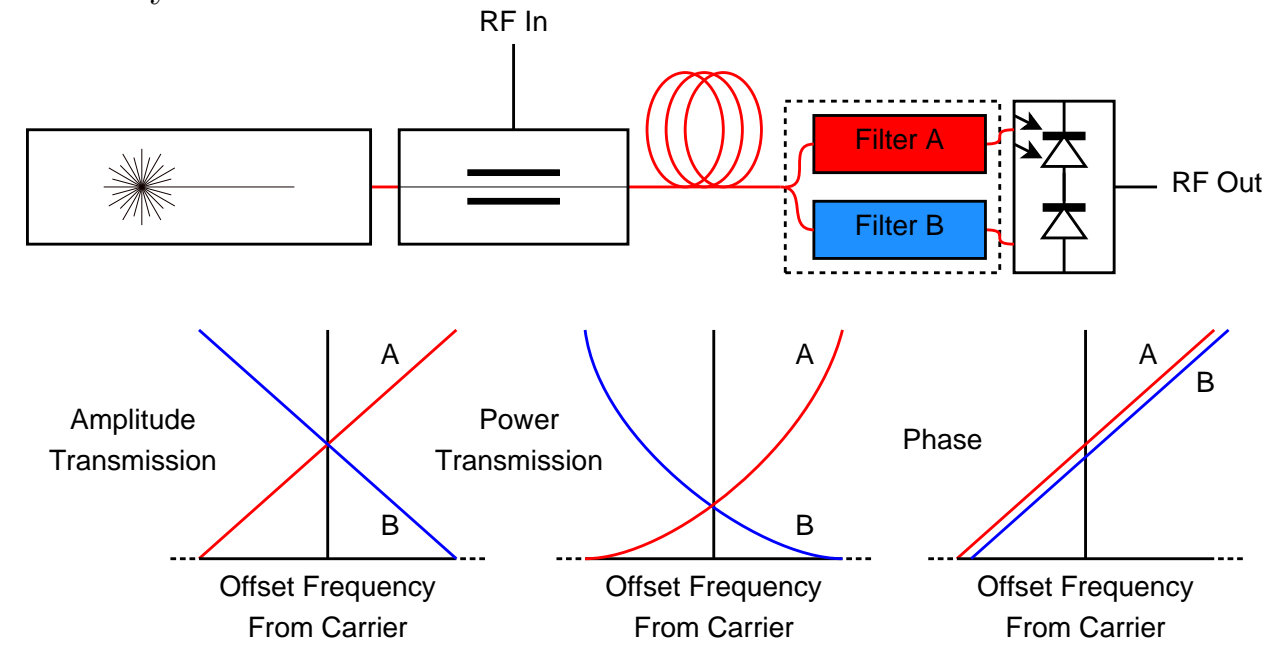

\subsection{History}

The work of Harris, [40], was the earliest use of a quadrature biased Mach Zehnder interferometer structure to discriminate optical FM. An interferometric path difference was created by passing the light through a birefringent crystal when the light's polarization was angled between the fast and slow axes of the crystal. It was noted by Harris that optimal FM to AM conversion occurs at the quadrature bias point. The technique was also applied to phase modulated light in [32]. Besides PM to AM discrimination, suppression of unwanted incident AM was done by applying a 180 degree phase shift to one of the two complementary polarization states at the output of the discriminator. The initial AM canceled when both polarization states, now with their PM in phase but AM 180 degrees out of phase, were detected at a single polarization-insensitive photodetector.

Another physical implementation of the MZI style discriminator using mirrors and beam splitters was suggested by [34]. In this case, balanced photodetection was used to cancel AM. Such an interferometer was experimentally verified by [41]. [34] also suggested the use of balanced detection for the birefringent crystal device of [40].

Concurrent to the development of direct frequency modulation of semiconductor lasers in works such as [42], [43] performed digital data transmission experiments using a Michelson interferometer to discriminate optical frequency shift keying (FSK).

The use of FM semiconductor lasers and discriminator detection was extended to transmitting subcarrier-multiplexed, analog signals for applications in cable television distribution. Experimental results for a Fabry-Perot discriminated, FM subcarriermultiplexed system were presented by [44]. An array of optical frequency modulated DFB lasers and a Fabry-Perot discriminator were used to transmit and demodulate a large number of microwave FM, analog video channels. A similar system was also used to transmit subcarrier-multiplexed, digital signals in [35].

Because analog links require high linearity and low noise, a number of authors have 
derived figures of merit for the performance of analog FM-DD links. [45] analyzed the frequency-dependent response of a link with a quadrature biased MZI discriminator subject to large modulation-depth AM and FM. [46] studied the intermodulation distortion for a Fabry-Perot discriminated link with a large number of channels, while taking into account both FM and IM on each channel. [17] derived figures of merit for the dynamic range of a phase modulated link with an MZI discriminator and balanced detection.

[47] studied a link with an arbitrary discriminator. The general formulae were applied to the particular cases of an MZI and a Fabry-Perot interferometer. However, the analysis was inaccurate since it looked at the system in terms of light intensity transmission through the interferometer, and ignored the coherence of the filtering. The transmission was expanded in terms of a Taylor series. The analysis assumed that derivatives of the transmission spectrum of the interferometer (in the Fourier-frequency domain) with respect to the instantaneous optical frequency were proportional to overall link nonlinearity. Similar (inaccurate) theoretical analyses using Taylor series were published by [48] and [49]. However, these papers did include new models for the nonlinearities in the lasers' FM and included the effects of residual IM.

To improve the linearity of an FM-DD link, many alternatives to the MachZehnder and Fabry-Perot interferometers have been suggested. In very early work, [33] proposed a linear-field discriminator using a network of birefringent crystals. The device was a tenth-order finite-impulse-response (FIR) filter. The series of crystals worked as a series of cascaded Mach Zehnder interferometers, and the network was equivalent to a lattice filter architecture. The filter coefficients chosen were the exponential Fourier series approximation to a triangular wave. The authors understood that linear demodulation, required for high fidelity signal transmission, could be accomplished with a discriminator that has a linear FM to AM transfer function, and that high-order filters could be used to implement this linear-field discriminator.

Except for the early work of [33], other "linearized" discriminators in the literature were designed such that the filter's optical intensity transmission ramped linearly with frequency offset from the carrier, rather than the field amplitude. These designs are not consistent with our theoretical link models. [50] and [51] proposed pairs of chirped fiber-Bragg gratings with either the index variation or chirp rate varied nonlinearly. [38] proposed a frequency discriminator based on an MZI with ring resonators in its arms. [52] suggested that the linearity of a Sagnac discriminator could be improved by adding ring resonators.

There have been recent experimental results for discriminators with intensity versus frequency offset ramps. None of these devices have demonstrated significant linearity improvement over a MZI . Design and experimental results from a microring structure implemented in a CMOS waveguide process were reported by [37, 53]. Experimental and theoretical results using fiber-Bragg gratings were presented in $[36,54-58]$. These experiments used pairs of complementary gratings designed to have a a transfer function whose intensity transmission ramped linearly with offset frequency from the carrier. The gratings were low-biased to perform carrier suppression. In $[56,58]$, the authors presented a clipping-free dynamic range limit for an 
FM-DD system. (In related work, [59, 60], the authors used Bragg gratings to convert phase modulation into single sideband modulation.) After a theoretical analysis, the authors later realized the limitations of their discriminator filter design, [57]:

[...] the ideal linear power reflectivity-versus-frequency curve does not result in an ideal half-wave rectification, as suggested by the simple time-domain view. Rather, in addition to the signal component, the output includes a dc component as well as a nonlinear distortion.

They explained the discrepancy, [36]:

The reason this intuition fails is that combining a time-domain view of the FM signal (instantaneous frequency, not averaged over time) with a frequency domain view of the FBG filter response is inconsistent with the frequency domain analysis $[\ldots]$

It is erroneous to think of the modulated signal in terms of its instantaneous frequency while looking at the frequency spectrum of the filter. The carrier is not really being swept along the ramp of the filter by the modulation, so considering it in the same way as, for example, the small-signal current to voltage relationship of an amplifier is not correct. In this work, I present complementary linear-field demodulation as a technique that can produce a microwave photonic link with low distortion.

\subsection{Analytical link analysis}

In this section, I derive figures-of-merit for a PM-DD or an FM-DD link that uses an arbitrary optical filter for discrimination, following my published work in [39]. This derivation is related to earlier theoretical work by [36], who published results for single-tone modulation. Follow-up work has been performed by [61], which consider links with partially coherent sources. I find expressions for the currents at each microwave frequency at the output of the link under a two-tone test. I take a smallmodulation-depth approximation. The standard definitions for the linearity figures of merit rely on this small signal approximation. I obtain expressions for the signalto-noise ration (SNR), second-order and third-order output intercept points (OIP2 and OIP3), spurious-free dynamic range (SFDR) and noise figure (NF). I apply these general formulae to the specific cases of the Mach Zehnder interferometer, a linear intensity ramp filter and complementary linear-field filters. For the linear-field filter, I derive the noise figure's dependence on the link's regime of operation and quantify the effect of filter curvature and the laser's residual IM on the distortion.

\subsubsection{Two tone derivation}

An optical signal that is phase or frequency modulated by two sinusoidal tones can be represented by the time varying electric field

$$
e_{\text {mod }}(t)=\kappa \sqrt{2 P_{\text {opt }}} \cos \left[2 \pi f_{c} t+\beta_{1} \sin \left(2 \pi f_{1} t\right)+\beta_{2} \sin \left(2 \pi f_{2} t\right)\right]
$$


where $P_{\text {opt }}$ is the rms optical power, $\kappa$ is a constant with units relating optical field and optical power such that $P_{\text {opt }}=\left\langle e(t)^{2}\right\rangle / \kappa^{2}, f_{c}$ is the frequency of the optical carrier, $f_{1}$ and $f_{2}$ are the modulation frequencies and $\beta_{1}$ and $\beta_{2}$ are the angle modulation depths. For PM, $\beta$ is the peak phase shift induced by the modulator. For a peak applied voltage of $V$, the peak phase shift is $\beta=\pi V / V_{\pi}(f)$, and the halfway voltage is generally frequency dependent. For FM, each modulation depth is equal to the maximum optical frequency deviation of the carrier induced by the modulation divided by the frequency of the modulation, $\beta=\delta_{f} / f$. The modulation of the light can be thought of in terms of variations in the instantaneous frequency of the light due to the applied signal. The optical frequency, or wavelength, varies sinusoidally in time. The instantaneous frequency of the light is given by the derivative of the phase of the light,

$$
\frac{1}{2 \pi} \frac{\partial}{\partial t}\left[2 \pi f_{c} t+\beta_{1} \sin \left(2 \pi f_{1} t\right)+\beta_{2} \sin \left(2 \pi f_{2} t\right)\right]=f_{c}+\delta_{f_{1}} \cos \left(2 \pi f_{1} t\right)+\delta_{f_{2}} \cos \left(2 \pi f_{2} t\right)
$$

The link generally has additional undesired residual IM and noise. The correction to the electric field is

$$
\begin{aligned}
e_{\text {mod }}(t)= & a(t)+\kappa \sqrt{2 P_{\text {opt }}[1+n(t)]} \\
& \cdot \sqrt{1+m_{1} \cos \left(2 \pi f_{1} t+\phi\right)+m_{2} \cos \left(2 \pi f_{2} t+\phi\right)} \\
& \cdot \cos \left[2 \pi f_{c} t+\beta_{1} \sin \left(2 \pi f_{1} t\right)+\beta_{2} \sin \left(2 \pi f_{2} t\right)+\varphi(t)\right]
\end{aligned}
$$

where $n(t)$ is the RIN of the source, $\varphi(t)$ is the phase noise of the source, $a(t)$ is the ASE noise from an optical amplifier, $m_{1}$ and $m_{2}$ represent the IM depths for the two tones and $\phi$ is the phase difference between the IM and the FM. The link will also amplify thermal noise present at the input.

In the next few equations, I expand the expression for the modulated electric field into its frequency components so that filtering can be expressed in the frequency domain. The residual IM depth and the intensity noise are assumed to be much smaller than the angle modulation, so the square root in (2.3) can be expanded using a Taylor series, yielding

$$
\begin{aligned}
e_{\text {mod }}(t) \approx & a(t)+\kappa \sqrt{2 P_{\text {opt }}} \\
& \cdot\left(1+\frac{1}{2} m_{1} \cos \left(2 \pi f_{1} t+\phi\right)+\frac{1}{2} m_{2} \cos \left(2 \pi f_{2} t+\phi\right)+\frac{1}{2} n(t)\right) \\
& \cdot \cos \left[2 \pi f_{c} t+\beta_{1} \sin \left(2 \pi f_{1} t\right)+\beta_{2} \sin \left(2 \pi f_{2} t\right)+\varphi(t)\right]
\end{aligned}
$$

Ignoring noise, this can be written using an angular addition trigonometric identity 
as

$$
\begin{aligned}
e_{\text {mod }}(t)= & \kappa \sqrt{2 P_{\text {opt }}} \operatorname{Re}\{ \\
& \cos \left[2 \pi f_{c} t+\beta_{1} \sin \left(2 \pi f_{1} t\right)+\beta_{2} \sin \left(2 \pi f_{2} t\right)\right] \\
& +\frac{1}{4} m_{1} \cos \left[2 \pi\left(f_{c}+f_{1}\right) t+\beta_{1} \sin \left(2 \pi f_{1} t\right)+\beta_{2} \sin \left(2 \pi f_{2} t\right)+\phi\right] \\
& +\frac{1}{4} m_{1} \cos \left[2 \pi\left(f_{c}-f_{1}\right) t+\beta_{1} \sin \left(2 \pi f_{1} t\right)+\beta_{2} \sin \left(2 \pi f_{2} t\right)-\phi\right] \\
& +\frac{1}{4} m_{2} \cos \left[2 \pi\left(f_{c}+f_{2}\right) t+\beta_{1} \sin \left(2 \pi f_{1} t\right)+\beta_{2} \sin \left(2 \pi f_{2} t\right)+\phi\right] \\
& \left.+\frac{1}{4} m_{2} \cos \left[2 \pi\left(f_{c}-f_{1}\right) t+\beta_{1} \sin \left(2 \pi f_{1} t\right)+\beta_{2} \sin \left(2 \pi f_{2} t\right)-\phi\right]\right\}
\end{aligned}
$$

The Jacobi-Anger expansion is given by $e^{i \beta \cos \theta}=\sum_{n=-\infty}^{\infty} j^{n} J_{n}(\beta) e^{i n \theta}$, where $j$ is the imaginary unit and $J_{n}(\beta)$ is a Bessel function of the first kind. Applying this formula, the expression in final form expands to

$$
\begin{aligned}
e_{\text {mod }}(t)= & \kappa \sqrt{2 P_{\text {opt }}} \operatorname{Re}\{ \\
& \sum_{n=-\infty}^{\infty} \sum_{p=-\infty}^{\infty} J_{n}\left(\beta_{1}\right) J_{p}\left(\beta_{2}\right) \exp \left[j 2 \pi\left(f_{c}+n f_{1}+p f_{2}\right) t\right] \\
& +\frac{1}{4} m_{1} \sum_{n=-\infty}^{\infty} \sum_{p=-\infty}^{\infty} J_{n}\left(\beta_{1}\right) J_{p}\left(\beta_{2}\right) \exp \left[j 2 \pi\left(f_{c}+[n+1] f_{1}+p f_{2}\right) t+j \phi\right] \\
& +\frac{1}{4} m_{1} \sum_{n=-\infty}^{\infty} \sum_{p=-\infty}^{\infty} J_{n}\left(\beta_{1}\right) J_{p}\left(\beta_{2}\right) \exp \left[j 2 \pi\left(f_{c}+[n-1] f_{1}+p f_{2}\right) t-j \phi\right] \\
& +\frac{1}{4} m_{2} \sum_{n=-\infty}^{\infty} \sum_{p=-\infty}^{\infty} J_{n}\left(\beta_{1}\right) J_{p}\left(\beta_{2}\right) \exp \left[j 2 \pi\left(f_{c}+n f_{1}+[p+1] f_{2}\right) t+j \phi\right] \\
& \left.+\frac{1}{4} m_{2} \sum_{n=-\infty}^{\infty} \sum_{p=-\infty}^{\infty} J_{n}\left(\beta_{1}\right) J_{p}\left(\beta_{2}\right) \exp \left[j 2 \pi\left(f_{c}+n f_{1}+[p-1] f_{2}\right) t-j \phi\right]\right\}
\end{aligned}
$$

An arbitrary optical filter is used on the link to convert the angle modulation to IM. With multiple detectors, we denote the transfer function seen by the field before each detector as $H^{z}(f)$ for the $\mathrm{z}^{\text {th }}$ of $\mathrm{Z}$ detectors. For example, $H^{1}\left(f_{c}\right)$ is the attenuation of the optical carrier seen at the first detector. The transfer function includes the splitting loss. For later convenience, I employ a shorthand notation for electric field transmission at each frequency in the optical spectrum that corresponds to an optical sideband around the carrier:

$$
h_{n, p}^{z} \equiv H^{z}\left(f_{c}+n f_{1}+p f_{2}\right)
$$

where $n$ and $p$ are integer indices and $H$ is the complex transfer function of the filter, representing its phase and amplitude response, including any insertion losses 
or optical amplifier gain. For example, $h_{0,0}$ is the field transmission for the optical carrier, and $h_{-1,0}$ is the transmission of the negative, first order sideband spaced $f_{1}$ away from the carrier.

The electric field after the filter at photodetector $z$ is

$$
\begin{aligned}
e_{\text {det }}^{z}(t)= & e_{\text {mod }}(t) * h^{z}(t) \\
= & \kappa \sqrt{2 P_{\text {opt }}} \operatorname{Re}\{ \\
& \sum_{n=-\infty}^{\infty} \sum_{p=-\infty}^{\infty} J_{n}\left(\beta_{1}\right) J_{p}\left(\beta_{2}\right) h_{n, p}^{z} \exp \left[j 2 \pi\left(f_{c}+n f_{1}+p f_{2}\right) t\right] \\
& +\frac{1}{4} m_{1} \sum_{n=-\infty}^{\infty} \sum_{p=-\infty}^{\infty} J_{n}\left(\beta_{1}\right) J_{p}\left(\beta_{2}\right) h_{n+1, p}^{z} \exp \left[j 2 \pi\left(f_{c}+[n+1] f_{1}+p f_{2}\right) t+j \phi\right] \\
& +\frac{1}{4} m_{1} \sum_{n=-\infty}^{\infty} \sum_{p=-\infty}^{\infty} J_{n}\left(\beta_{1}\right) J_{p}\left(\beta_{2}\right) h_{n-1, p}^{z} \exp \left[j 2 \pi\left(f_{c}+[n-1] f_{1}+p f_{2}\right) t-j \phi\right] \\
& +\frac{1}{4} m_{2} \sum_{n=-\infty}^{\infty} \sum_{p=-\infty}^{\infty} J_{n}\left(\beta_{1}\right) J_{p}\left(\beta_{2}\right) h_{n, p+1}^{z} \exp \left[j 2 \pi\left(f_{c}+n f_{1}+[p+1] f_{2}\right) t+j \phi\right] \\
& \left.+\frac{1}{4} m_{2} \sum_{n=-\infty}^{\infty} \sum_{p=-\infty}^{\infty} J_{n}\left(\beta_{1}\right) J_{p}\left(\beta_{2}\right) h_{n, p-1}^{z} \exp \left[j 2 \pi\left(f_{c}+n f_{1}+[p-1] f_{2}\right) t-j \phi\right]\right\}
\end{aligned}
$$

The indices of each infinite sum can be renumbered to obtain

$$
\begin{aligned}
e_{\text {det }}^{z}(t)= & \kappa \sqrt{2 P_{\text {opt }}} \operatorname{Re}\{ \\
& \sum_{n=-\infty}^{\infty} \sum_{p=-\infty}^{\infty} J_{n}\left(\beta_{1}\right) J_{p}\left(\beta_{2}\right) h_{n, p}^{z} \exp \left[j 2 \pi\left(f_{c}+n f_{1}+p f_{2}\right) t\right] \\
& +\frac{1}{4} m_{1} \sum_{n=-\infty}^{\infty} \sum_{p=-\infty}^{\infty} J_{n-1}\left(\beta_{1}\right) J_{p}\left(\beta_{2}\right) h_{n, p}^{z} \exp \left[j 2 \pi\left(f_{c}+n f_{1}+p f_{2}\right) t+j \phi\right] \\
& +\frac{1}{4} m_{1} \sum_{n=-\infty}^{\infty} \sum_{p=-\infty}^{\infty} J_{n+1}\left(\beta_{1}\right) J_{p}\left(\beta_{2}\right) h_{n, p}^{z} \exp \left[j 2 \pi\left(f_{c}+n f_{1}+p f_{2}\right) t-j \phi\right] \\
& +\frac{1}{4} m_{2} \sum_{n=-\infty}^{\infty} \sum_{p=-\infty}^{\infty} J_{n}\left(\beta_{1}\right) J_{p-1}\left(\beta_{2}\right) h_{n, p}^{z} \exp \left[j 2 \pi\left(f_{c}+n f_{1}+p f_{2}\right) t+j \phi\right] \\
& \left.+\frac{1}{4} m_{2} \sum_{n=-\infty}^{\infty} \sum_{p=-\infty}^{\infty} J_{n}\left(\beta_{1}\right) J_{p+1}\left(\beta_{2}\right) h_{n, p}^{z} \exp \left[j 2 \pi\left(f_{c}+n f_{1}+p f_{2}\right) t-j \phi\right]\right\}
\end{aligned}
$$

This simplifies to a compact expression for the signal after the filter in terms of its frequency components,

$$
e_{\text {det }}^{z}(t)=\kappa \sqrt{2 P_{\text {opt }}} \operatorname{Re}\left\{\sum_{n=-\infty}^{\infty} \sum_{p=-\infty}^{\infty} j_{n, p}^{z} \exp \left[j 2 \pi\left(f_{c}+n f_{1}+p f_{2}\right) t\right]\right\}
$$


where I define

$$
\begin{aligned}
j_{n, p}^{z} \equiv & h_{n, p}^{z}\left\{J_{n}\left(\beta_{1}\right) J_{p}\left(\beta_{2}\right)\right. \\
& +\frac{1}{4} m_{1}\left[J_{n-1}\left(\beta_{1}\right) e^{j \phi}+J_{n+1}\left(\beta_{1}\right) e^{-j \phi}\right] J_{p}\left(\beta_{2}\right) \\
& \left.+\frac{1}{4} m_{2} J_{n}\left(\beta_{1}\right)\left[J_{p-1}\left(\beta_{2}\right) e^{j \phi}+J_{p+1}\left(\beta_{1}\right) e^{-j \phi}\right]\right\}
\end{aligned}
$$

The electric field is incident upon a photodetector at the termination of a fiber-optic link. The photodetector is assumed to be an ideal square-law detector operating in its linear regime with responsivity $\Re$. The photocurrent is

$$
i^{z}(t)=\Re P_{o p t}\left\{\sum_{n=-\infty}^{\infty} \sum_{p=-\infty}^{\infty} \sum_{g=-\infty}^{\infty} \sum_{k=-\infty}^{\infty} j_{n, p}^{z} j_{g, k}^{z *} \exp \left[j 2 \pi\left([n-g] f_{1}+[p-k] f_{2}\right) t\right]\right\}
$$

This can be split up into the de term, harmonics of $f_{1}$, harmonics of $f_{2}$ and mixtures between $f_{1}$ and $f_{2}$.

$$
\begin{aligned}
i^{z}(t)= & \Re P_{o p t}\left\{\sum_{g=-\infty}^{\infty} \sum_{k=-\infty}^{\infty}\left|j_{g, k}^{z}\right|^{2}\right. \\
& +\sum_{n=-\infty}^{\infty, n \neq g} \sum_{g=-\infty}^{\infty} \sum_{k=-\infty}^{\infty} j_{n, k}^{z} j_{g, k}^{z *} \exp \left[j 2 \pi[n-g] f_{1} t\right] \\
& +\sum_{p=-\infty}^{\infty, p \neq k} \sum_{g=-\infty}^{\infty} \sum_{k=-\infty}^{\infty} j_{g, p}^{z} j_{g, k}^{z *} \exp \left[j 2 \pi[p-k] f_{2} t\right] \\
& \left.+\sum_{n=-\infty}^{\infty, n \neq g} \sum_{p=-\infty}^{\infty, p \neq k} \sum_{g=-\infty}^{\infty} \sum_{k=-\infty}^{\infty} j_{n, p}^{z} j_{g, k}^{z *} \exp \left[j 2 \pi\left([n-g] f_{1}+[p-k] f_{2}\right) t\right]\right\}
\end{aligned}
$$

The indices of each infinite sum can be renumbered to obtain

$$
\begin{aligned}
i^{z}(t)= & \Re P_{o p t}\left\{\sum_{g=-\infty}^{\infty} \sum_{k=-\infty}^{\infty}\left|j_{g, k}^{z}\right|^{2}\right. \\
& +\sum_{n=-\infty}^{\infty, n \neq 0} \sum_{g=-\infty}^{\infty} \sum_{k=-\infty}^{\infty} j_{n+g, k}^{z} j_{g, k}^{z *} \exp \left[j 2 \pi n f_{1} t\right] \\
& +\sum_{p=-\infty}^{\infty, p \neq 0} \sum_{g=-\infty}^{\infty} \sum_{k=-\infty}^{\infty} j_{g, p+k}^{z} j_{g, k}^{z *} \exp \left[j 2 \pi p f_{2} t\right] \\
& \left.+\sum_{n=-\infty}^{\infty, n \neq 0} \sum_{p=-\infty}^{\infty, p \neq 0} \sum_{g=-\infty}^{\infty} \sum_{k=-\infty}^{\infty} j_{n+g, p+k}^{z} j_{g, k}^{z *} \exp \left[j 2 \pi\left(n f_{1}+p f_{2}\right) t\right]\right\}
\end{aligned}
$$

The double infinite sums over $n$ and $p$ are rewritten as singly infinite sums, and the 
sums over negative integers have their signs flipped giving

$$
\begin{aligned}
i^{z}(t)= & \Re P_{o p t}\left\{\sum_{g=-\infty}^{\infty} \sum_{k=-\infty}^{\infty}\left|j_{g, k}^{z}\right|^{2}\right. \\
& +\sum_{n=1}^{\infty} \sum_{g=-\infty}^{\infty} \sum_{k=-\infty}^{\infty}\left(j_{n+g, k}^{z} j_{g, k}^{z *} \exp \left[j 2 \pi n f_{1} t\right]+j_{-n+g, k}^{z} j_{g, k}^{z *} \exp \left[-j 2 \pi n f_{1} t\right]\right) \\
& +\sum_{p=1}^{\infty} \sum_{g=-\infty}^{\infty} \sum_{k=-\infty}^{\infty}\left(j_{g, p+k}^{z} j_{g, k}^{z *} \exp \left[j 2 \pi p f_{2} t\right]+j_{g,-p+k}^{z} j_{g, k}^{z *} \exp \left[-j 2 \pi p f_{2} t\right]\right) \\
& +\sum_{n=1}^{\infty} \sum_{p=1}^{\infty} \sum_{g=-\infty}^{\infty} \sum_{k=-\infty}^{\infty} \\
& \left(j_{n+g, p+k}^{z} j_{g, k}^{z *} \exp \left[j 2 \pi\left(n f_{1}+p f_{2}\right) t\right]+j_{-n+g,-p+k}^{z} j_{g, k}^{z *} \exp \left[-j 2 \pi\left(n f_{1}+p f_{2}\right) t\right]\right. \\
& \left.\left.+j_{n+g,-p+k}^{z} j_{g, k}^{z *} \exp \left[j 2 \pi\left(n f_{1}-p f_{2}\right) t\right]+j_{-n+g, p+k}^{z} j_{g, k}^{z *} \exp \left[-j 2 \pi\left(n f_{1}-p f_{2}\right) t\right]\right)\right\}
\end{aligned}
$$

A number added to its complex conjugate is twice the real part. With this simplification, this arranges to a final expression for the photodetector output given an arbitrary filter:

$$
\begin{aligned}
i^{z}(t)= & \Re P_{\text {opt }} \operatorname{Re}\left\{\sum_{g=-\infty}^{\infty} \sum_{k=-\infty}^{\infty}\left|j_{g, k}^{z}\right|^{2}\right. \\
& +2 \sum_{n=1}^{\infty} \sum_{g=-\infty}^{\infty} \sum_{k=-\infty}^{\infty} j_{n+g, k}^{z} j_{g, k}^{z *} \exp \left[j 2 \pi n f_{1} t\right] \\
& +2 \sum_{p=1}^{\infty} \sum_{g=-\infty}^{\infty} \sum_{k=-\infty}^{\infty} j_{g, p+k}^{z} j_{g, k}^{z *} \exp \left[j 2 \pi p f_{2} t\right] \\
& +2 \sum_{n=1}^{\infty} \sum_{p=1}^{\infty} \sum_{g=-\infty}^{\infty} \sum_{k=-\infty}^{\infty} j_{n+g, p+k}^{z} j_{g, k}^{z *} \exp \left[j 2 \pi\left(n f_{1}+p f_{2}\right) t\right] \\
& \left.+2 \sum_{n=1}^{\infty} \sum_{p=1}^{\infty} \sum_{g=-\infty}^{\infty} \sum_{k=-\infty}^{\infty} j_{n+g,-p+k}^{z} j_{g, k}^{z *} \exp \left[j 2 \pi\left(n f_{1}-p f_{2}\right) t\right]\right\}
\end{aligned}
$$

The double-sum over indices $g$ and $k$ gives the contribution of each pair of optical sidebands that beat together to produce the rf photocurrent. In this form, the current is separated into different frequency components which are indicated by the summation indices $n$ and $p$. The first term, where $n$ and $p$ are both identically zero, gives the dc. The second term, a summation over the index $n$, gives the fundamental tone at frequency $f_{1}$ and its harmonics. The third term, a summation over the index $p$, gives the fundamental tone at frequency $f_{2}$ and its harmonics. The fourth term is the sum frequencies produced by the mixing, and the fifth term is the difference frequencies produced by the mixing. 


\subsubsection{Small signal approximation}

For small modulation depth, $\beta \ll 1$, and no residual IM, $m=0$, the Bessel functions can be approximated by $J_{0}(\beta) \approx 1$ and $J_{n}(\beta) \approx(\beta / 2)^{|n|} /|n|$ !, for positive $n$, noting that $J_{-n}(\beta)=(-1)^{n} J_{n}(\beta)$. Keeping terms of lowest polynomial order, the current simplifies to the following equation (2.10). This equation gives the small signal approximation for any frequency:

$$
\begin{aligned}
i^{z}(t)= & \Re P_{o p t} \operatorname{Re}\left\{\left|h_{0,0}^{z}\right|^{2}\right. \\
& +2 \sum_{n=1}^{\infty} \sum_{g=0}^{n} \frac{\beta_{1}^{n}}{2^{n}} \frac{(-1)^{g}}{(n-g) ! g !} h_{n-g, 0}^{z} h_{-g, 0}^{z *} \exp \left[j 2 \pi n f_{1} t\right] \\
& +2 \sum_{p=1}^{\infty} \sum_{k=0}^{p} \frac{\beta_{2}^{p}}{2^{p}} \frac{(-1)^{k}}{(p-k) ! k !} h_{0, p-k}^{z} h_{0,-k}^{z *} \exp \left[j 2 \pi p f_{2} t\right] \\
& +2 \sum_{n=1}^{\infty} \sum_{p=1}^{\infty} \sum_{g=0}^{n} \sum_{k=0}^{p} \frac{\beta_{1}^{n} \beta_{2}^{p}}{2^{n+p}} \frac{(-1)^{g+k}}{(n-g) ! g !(p-k) ! k !} h_{n-g, p-k}^{z} h_{-g,-k}^{z *} \exp \left[j 2 \pi\left(n f_{1}+p f_{2}\right) t\right] \\
& \left.+2 \sum_{n=1}^{\infty} \sum_{p=1}^{\infty} \sum_{g=0}^{n} \sum_{k=0}^{p} \frac{\beta_{1}^{n} \beta_{2}^{p}}{2^{n+p}} \frac{(-1)^{p+g+k}}{(n-g) ! g !(p-k) ! k !} h_{n-g,-p+k}^{z} h_{-g, k}^{z *} \exp \left[j 2 \pi\left(n f_{1}-p f_{2}\right) t\right]\right\}
\end{aligned}
$$

There are four current components of interest. The amplitude of the dc, as should be expected, is proportional to the optical power in the optical carrier after the filter. The current at the fundamental frequency $f_{1}$ is linearly proportional to the modulation depth. It depends on the negative and positive first-order sidebands beating with the optical carrier. The current at the second-harmonic frequency $2 f_{1}$ has a quadratic relationship to modulation depth. It depends on the second-order sidebands beating with the optical carrier, as well as the first-order sidebands beating with each other. The current produced at the difference frequency $2 f_{1}-f_{2}$ is a third-order intermodulation product. These currents are

$$
\begin{aligned}
i_{d c}^{z} & =\Re P_{o p t} X_{0}^{z} \\
i_{f_{1}}^{z} & =\Re P_{o p t} \beta_{1} \operatorname{Re}\left\{X_{1}^{z} \exp \left[j 2 \pi f_{1} t\right]\right\} \\
i_{2 f_{1}}^{z} & =\Re P_{o p t} \frac{1}{4} \beta_{1}^{2} \operatorname{Re}\left\{X_{2}^{z} \exp \left[j 4 \pi f_{1} t\right]\right\} \\
i_{2 f_{1}-f_{2}}^{z} & =\Re P_{o p t} \frac{1}{8} \beta_{1}^{2} \beta_{2} \operatorname{Re}\left\{X_{3}^{z} \exp \left[j 2 \pi\left(2 f_{1}-f_{2}\right) t\right]\right\}
\end{aligned}
$$

where for convenience, I define the following complex constants, which I will call link 
distortion constants:

$$
\begin{aligned}
X_{0}^{z}= & h_{0,0}^{z} h_{0,0}^{z *} \\
X_{1}^{z}= & h_{1, \sum_{k=-\infty}^{z} j_{n+g,-p+k}^{z} j_{g, k}^{z *} 0} h_{0,0}^{z *}-h_{0,0}^{z} h_{-1,0}^{z *} \\
Y_{1}^{z}= & h_{1,0}^{z} h_{0,0}^{z *}+h_{0,0}^{z} h_{-1,0}^{z *} \\
X_{2}^{z}= & h_{2,0}^{z} h_{0,0}^{z *}-2 h_{1,0}^{z} h_{-1,0}^{z *}+h_{0,0}^{z} h_{-2,0}^{z *} \\
X_{3}^{z}= & -h_{2,-1}^{z} h_{0,0}^{z *}+h_{2,0}^{z} h_{0,1}^{z *}+2 h_{1,-1}^{z} h_{-1,0}^{z *} \\
& +h_{0,0}^{z} h_{-2,1}^{z *}-h_{0,-1}^{z} h_{-2,0}^{z *}-2 h_{1,0}^{z} h_{-1,1}^{z *}
\end{aligned}
$$

For a balanced detector system, the currents subtract from each other. The link constants for each branch can be subtracted from each other such that $X_{0} \equiv X_{0}^{1}-X_{0}^{2}$, $X_{1} \equiv X_{1}^{1}-X_{1}^{2}$, etc. Each rf photocurrent outputs an rms power, which is proportional to the square of the dc current, into the load impedance, $Z_{\text {out }}$. The powers for the signal, second harmonic, and intermodulation distortion are as follows:

$$
\begin{aligned}
P_{f_{1}} & =\frac{1}{2}\left|Z_{\text {out }}\right| \Re^{2} P_{\text {opt }}^{2} \beta_{1}^{2}\left|X_{1}\right|^{2} \\
P_{2 f_{1}} & =\frac{1}{32}\left|Z_{\text {out }}\right| \Re^{2} P_{\text {opt }}^{2} \beta_{1}^{4}\left|X_{2}\right|^{2} \\
P_{2 f_{1}-f_{2}} & =\frac{1}{128}\left|Z_{\text {out }}\right| \Re^{2} P_{\text {opt }}^{2} \beta_{1}^{4} \beta_{2}^{2}\left|X_{3}\right|^{2}
\end{aligned}
$$

In this section, I have derived closed form expressions for the photocurrents at different frequencies at the output of a filtered FM link. A general result has been given in (2.9) which includes residual intensity modulation, and can be solved to arbitrary precision by taking a large number of terms in the infinite sum. A small signal approximation, (2.10), gives the output current at any frequency component of interest. Expressions for the photocurrent at the fundamental, second harmonic and third order intermodulation distortion have been derived, which will be useful in expressing figures of merit for distortion and dynamic range.

\subsubsection{Gain}

For an PM link, the modulator is driven by an applied voltage. The peak input voltage, $V_{i n}$ produces an rms input power $P_{i n}$ when delivered to a load impedance $Z_{\text {in }}$ such that $\beta_{1}^{2}=\left(\pi V_{i n} / V_{\pi}\right)^{2}=\pi^{2} 2 P_{i n}\left|Z_{i n}\right| / V_{\pi}^{2}$. The output signal is given by $(2.20)$. The gain is therefore

$$
G_{P M}=\left|Z_{\text {in }}\right|\left|Z_{\text {out }}\right|\left(\frac{\pi \Re P_{\text {opt }}}{V_{\pi}}\left|X_{1}\right|\right)^{2}
$$

For an FM link, the modulation efficiency of a current modulated FM laser is $\eta$, in units of $\mathrm{Hz} / \mathrm{A}$, typically of the order of a few hundred $\mathrm{MHz}$ per $\mathrm{mA}$. The peak input current, $i_{\text {in }}$ gives a modulation of $\delta_{f_{1}}^{2}=\left(\eta i_{i n}\right)^{2}=2 \eta^{2} P_{i n} /\left|Z_{i n}\right|$. The gain is therefore

$$
G_{F M}=\frac{\left|Z_{\text {out }}\right|}{\left|Z_{\text {in }}\right|}\left(\frac{\eta \Re P_{\text {opt }}}{f_{1}}\left|X_{1}\right|\right)^{2}
$$




\subsubsection{RF noise figure}

In this section, I derive the signal to noise ratio (SNR) for the small signal approximation of an arbitrary link and the noise figure. A passive link with no amplification will be considered, so the primary noises seen at the detector are shot, thermal, phase and RIN. The shot noise spectral density is proportional to $q$, the elementary charge, and to the total dc from the photodetectors, $i_{d c}=\Re P_{o p t} \sum\left|X_{0}^{z}\right|$. The thermal noise spectral density is equal to the product Boltzmann's constant, $k_{B}$, and the temperature, $T_{K}$.

$$
\begin{aligned}
& S_{\text {sn }}=2 q i_{d c}\left|Z_{\text {out }}\right| \\
& S_{\text {tn }}=k_{B} T_{K}
\end{aligned}
$$

Assuming a Lorentzian model for the laser's spectral line, the phase noise on the optical carrier is white noise with spectral density proportional to the laser's 3-dB linewidth, $\triangle \nu$ [62]. The phase fluctuations are converted to intensity fluctuations by the filter in the same manner as it converts the modulation. The average phase fluctuations in a small bandwidth near some frequency, $f$, are

$$
\left\langle\varphi(t)^{2}\right\rangle \approx \frac{\triangle \nu}{\pi} \frac{\triangle f}{f^{2}}
$$

Near the first modulation frequency, $f_{1}$, the power spectral density of the phase noise is

$$
S_{\text {pn }} \approx\left|Z_{\text {out }}\right| \Re^{2} P_{\text {opt }}^{2} \frac{\Delta \nu}{\pi f_{1}^{2}}\left|X_{1}\right|^{2}
$$

The modulation is assumed to be below the relaxation frequency of the laser, so the RIN is modeled as white noise. The power spectral density of the noise at the output, near the modulation frequency is

$$
S_{\text {in }} \approx \frac{1}{4}\left|Z_{\text {out }}\right| \Re^{2} P_{\text {opt }}^{2} \frac{\left\langle n(t)^{2}\right\rangle}{B}\left|Y_{1}\right|^{2}
$$

where $B$ is the bandwidth in $\mathrm{Hz}$. The total noise power is

$$
P_{\text {noise }} \approx\left(S_{s n}+S_{t n}+S_{p n}+S_{\text {in }}\right) B
$$

The noise figure is given by relation to the gain, given in (2.23) and (2.24), and for a thermal noise limited input as

$$
N F=1+\frac{1}{G}+\frac{\left(S_{s n}+S_{p n}+S_{i n}\right)}{G k_{B} T_{K}}
$$

For large positive link gain, approximations to the noise figure expressions for shot and phase noise limiting are given in Table 2.1 on page 25.

It is important to warn that the effect of noise caused by optical amplification has not been explicitly included in this analysis. It is likely that optical amplification, such as an EDFA, will be used in high performance PM/FM-DD link architectures since the NF and spurious free dynamic range scale with the optical power. The noise degradation from the laser's intrinsic noise by the amplifiers must be included in the quantity provided for the laser phase and amplitude noise. 
Table 2.1: Approximations to the noise figure expressions for arbitrarily filtered links. These assume large positive gain with either shot or phase noise limited noise figures. Shot noise limits occur for moderate optical powers and phase noise limit occurs for much larger optical powers. These approximations are not valid if the link attenuates the rf power.

\begin{tabular}{|c|c|c|}
\hline & PM & FM \\
\hline \hline Shot noise SNR & $\frac{\Re P_{\text {opt }} \beta_{1}^{2}\left|X_{1}\right|^{2}}{4 q B \sum\left|X_{0}^{z}\right|}$ & $\frac{\Re P_{\text {opt }} \delta_{f_{1}}^{2}\left|X_{1}\right|^{2}}{4 q B f_{1}^{2} \sum\left|X_{0}^{z}\right|}$ \\
\hline Phase noise SNR & $\frac{\pi \beta_{1}^{2} f_{1}^{2}}{2 \Delta \nu B}$ & $\frac{\pi \delta_{f_{1}}^{2}}{2 \Delta \nu B}$ \\
\hline Gain & $\left|Z_{\text {in }}\right|\left|Z_{\text {out }}\right|\left(\frac{\pi \Re P_{\text {opt }}}{V_{\pi}}\left|X_{1}\right|\right)^{2}$ & $\frac{\left|Z_{\text {out }}\right|}{\left|Z_{\text {in }}\right|}\left(\frac{\eta \Re P_{\text {opt }}}{f_{1}}\left|X_{1}\right|\right)^{2}$ \\
\hline Shot noise NF & $1+\frac{2 q V_{\pi}^{2} \sum\left|X_{0}^{z}\right|}{\left|Z_{\text {in }}\right| \Re P_{\text {opt }} \pi^{2}\left|X_{1}\right|^{2} k_{B} T_{K}}$ & $1+\frac{\left|Z_{i n}\right| f_{1}^{2} 2 q \sum\left|X_{0}^{z}\right|}{\Re P_{\text {opt }} \eta^{2}\left|X_{1}\right|^{2} k_{B} T_{K}}$ \\
\hline Phase noise NF & $1+\frac{\Delta \nu V_{\pi}^{2}}{\left|Z_{\text {in }}\right| \pi^{3} f_{1}^{2} k_{B} T_{K}}$ & $1+\frac{\left|Z_{i n}\right| \Delta \nu}{\eta^{2} \pi k_{B} T_{K}}$ \\
\hline
\end{tabular}

\subsubsection{Distortion}

The signal distortion caused by the link can be described by the output power at frequencies that are harmonics and mixing terms of the modulation frequencies. For now, I assume there is no residual IM, and assume two modulation tones have equal modulation depth, $\beta=\beta_{1}=\beta_{2}$ for PM, or $\delta_{f}=\delta_{f_{1}}=\delta_{f_{2}}$ for FM. The second-order output intercept point (OIP2) and third-order output intercept point (OIP3) for PM and FM are calculated in the table below.

\subsubsection{Spurious free dynamic range}

The spurious free dynamic range (SFDR) is defined as the SNR at the maximum usable modulation depth. This can be defined when either the second-order or thirdorder distortion products breach the noise floor. The second harmonic is equal to the shot noise power at modulation depth $\beta_{1}^{2}=\frac{8}{\left|X_{2}\right|} \sqrt{\frac{q B \sum\left|X_{0}^{z}\right|}{\Re P_{o p t}}}$ and to the phase noise power at modulation depth $\beta_{1}^{2}=4 \sqrt{\frac{2 \triangle \nu B}{\pi}}\left|X_{1}\right| / f_{1}\left|X_{2}\right|$. The IMD3 is equal to the shot noise power at modulation depth $\beta_{1}^{2}=\left(\frac{256 q B \sum\left|X_{0}^{z}\right|}{\Re P_{o p t}\left|X_{3}\right|^{2}}\right)^{1 / 3}$ and to the phase noise noise power at modulation depth $\beta_{1}^{2}=\left(\frac{128 \Delta \nu B\left|X_{1}\right|^{2}}{\pi f_{1}^{2}\left|X_{3}\right|^{2}}\right)^{1 / 3}$. Using the previously calculated expressions for the SNR, the spurious free dynamic ranges are compiled in Table 2.2. In addition, the third-order limited SFDR could also be calculated given the noise figure, gain, and output intercept points in $\mathrm{dB}$ units by the expression $S F D R=\frac{2}{3}\left(O I P 3-G+174 \frac{\mathrm{dBm}}{\mathrm{Hz}}-10 \log _{10}(B)-N F\right)$. These figures-of-merit are often defined with respect to $1 \mathrm{~Hz}$ bandwidth. They generally depend on the 
Table 2.2: General expressions for OIP2, OIP3, and spurious free dynamic range for an abitrarily filtered link with either phase or frequency modulation and direct detection given in terms of the link distortion constants. SFDR is limited by either shot or phase noise, and second-order or third-order distortion.

\begin{tabular}{|c|c|c|}
\hline & PM & FM \\
\hline \hline OIP2 & $8\left|Z_{\text {out }}\right| \Re^{2} P_{\text {opt }}^{2} \mid \frac{\left|X_{1}\right|^{4}}{\left|X_{2}\right|^{2}}$ & $8\left|Z_{\text {out }}\right| \Re^{2} P_{\text {opt }}^{2} \mid \frac{\left|X_{1}\right|^{4}}{\left|X_{2}\right|^{2}}$ \\
\hline Shot noise $S F D R_{2}$ & $2 \frac{\left|X_{1}\right|^{2}}{\left|X_{2}\right|} \sqrt{\frac{\Re P_{\text {opt }}}{q B \sum\left|X_{0}^{z}\right|}}$ & $2 \frac{\left|X_{1}\right|^{2}}{\left|X_{2}\right|} \sqrt{\frac{\Re P_{\text {opt }}}{q B \sum\left|X_{0}^{z}\right|}}$ \\
\hline Phase noise $S F D R_{2}$ & $2 \frac{\left|X_{1}\right|}{\left|X_{2}\right|} f_{1} \sqrt{\frac{2 \pi}{\Delta \nu B}}$ & $2 \frac{\left|X_{1}\right|}{\left|X_{2}\right|} f_{1} \sqrt{\frac{2 \pi}{\Delta \nu B}}$ \\
\hline OIP3 & $4\left|Z_{\text {out }}\right| \Re^{2} P_{\text {opt }}^{2} \frac{\left|X_{1}\right|^{3}}{\left|X_{3}\right|}$ & $4\left|Z_{\text {out }}\right| \Re^{2} P_{\text {opt }}^{2} \frac{f_{2}}{f_{1}} \frac{\left|X_{1}\right|^{3} \mid}{\left|X_{3}\right|}$ \\
\hline Shot noise $S F D R_{3}$ & $\left|X_{1}\right|^{2}\left(\frac{\Re P_{\text {opt }} 2}{\left|X_{3}\right| q B \sum\left|X_{0}^{z}\right|}\right)^{2 / 3}$ & $\left|X_{1}\right|^{2}\left(\frac{f_{2} \Re P_{\text {opt }} 2}{f_{1}\left|X_{3}\right| q B \sum\left|X_{0}^{z}\right|}\right)^{2 / 3}$ \\
\hline Phase noise $S F D R_{3}$ & $\left(\frac{4 f_{1}^{2} \pi\left|X_{1}\right|}{\Delta \nu B\left|X_{3}\right|}\right)^{2 / 3}$ & $\left(\frac{4 f_{1} f_{2} \pi\left|X_{1}\right|}{\Delta \nu B\left|X_{3}\right|}\right)^{2 / 3}$ \\
\hline
\end{tabular}

particular modulation frequencies chosen. Maximizing the ratios of $\left|X_{1}\right| /\left|X_{2}\right|$ and $\left|X_{1}\right| /\left|X_{3}\right|$ will improve the dynamic range of the link.

\subsection{Mach-Zehnder interferometer}

The simplest filter used as a discriminator is an asymmetrical Mach-Zehnder interferometer (a-MZI). I derive figures of merit for this link in order to verify the general theory against previously published results. One arm of the interferometer has a time shift with respect to the second arm, denoted by $\tau$. We assume $50 \%$ coupling ratios and quadrature bias, obtained by choosing the carrier frequency and time delay.

The filter transfer functions seen by the two output branches of the Mach Zehnder interferometer are

$$
\begin{aligned}
& h_{n, p}^{1}=\frac{1}{2}-\frac{j}{2} \exp \left[-j 2 \pi\left(n f_{1}+p f_{2}\right) \tau\right] \\
& h_{n, p}^{2}=\frac{j}{2}-\frac{1}{2} \exp \left[-j 2 \pi\left(n f_{1}+p f_{2}\right) \tau\right]
\end{aligned}
$$

By taking the absolute value squared of either transfer function, one can see that its intensity response is the familiar raised sinusoid and it is quadrature biased with half the carrier power transmitted to each branch.

Using the transfer functions, I evaluate the link distortion constants for both 
branches:

$$
\begin{aligned}
X_{0}^{1} & =\frac{1}{2} \\
X_{1}^{1} & =\frac{1}{2} j\left(1-\exp \left[-j 2 \pi f_{1} \tau\right]\right) \\
Y_{1}^{1} & =\frac{1}{2}\left(1+\exp \left[-j 2 \pi f_{1} \tau\right]\right) \\
X_{2}^{1} & =0 \\
X_{3}^{1} & =-4 \sin ^{2}\left[\pi f_{1} \tau\right] \sin \left[\pi f_{2} \tau\right] \exp \left[-j \pi\left(2 f_{1}-f_{2}\right) \tau\right]
\end{aligned}
$$

and

$$
\begin{aligned}
& X_{0}^{2}=\frac{1}{2} \\
& X_{1}^{2}=-j \frac{1}{2}\left(1-\exp \left[-j 2 \pi f_{1} \tau\right]\right) \\
& Y_{1}^{2}=\frac{1}{2}\left(1+\exp \left[-j 2 \pi f_{1} \tau\right]\right) \\
& X_{2}^{2}=0 \\
& X_{3}^{2}=4 \sin ^{2}\left[\pi f_{1} \tau\right] \sin \left[\pi f_{2} \tau\right] \exp \left[-j \pi\left(2 f_{1}-f_{2}\right) \tau\right]
\end{aligned}
$$

As expected for an MZI at quadrature, I find that there is no second-harmonic so that OIP2 is infinite. For the FM link, we choose a short time delay such that approximation $f_{1} \tau, f_{2} \tau \ll 1$ is valid. The absolute value of the other coefficients after the balanced detection are

$$
\begin{aligned}
& \left|X_{1}\right|=2\left|\sin \left(\pi f_{1} \tau\right)\right| \approx 2 \pi f_{1} \tau \\
& \left|X_{3}\right|=8 \sin ^{2}\left(\pi f_{1} \tau\right)\left|\sin \left(\pi f_{2} \tau\right)\right| \approx 8 \pi^{3} f_{1}^{2} f_{2} \tau^{3}
\end{aligned}
$$

A summary of the figures of merit are given in the table below. The same results are found by [17], which supports the general analysis. The important result from [17] was that the shot noise limited spurious free dynamic range of the PM-MZI link is identical to that of a Mach Zehnder modulated IM-DD link.

\subsection{Complementary linear-field demodulation}

In this section, I discuss filter transfer functions that allow for highly linear discrimination. I find that the ideal system has two filters with ramps of electric field transmission versus frequency, and linear phase.

A number of groups have proposed or built optical filters that have a transfer function linear in optical intensity versus frequency and small group delay. Within one-half period, the transfer function can be represented by

$$
h_{n, p}=\sqrt{A\left(f_{b}+n f_{1}+p f_{2}\right)} \exp \left[-j 2 \pi\left(f_{b}+n f_{1}+p f_{2}\right) \tau\right]
$$


Table 2.3: Figures of merit for an PM-DD link with an a-MZI and balanced detection.

\begin{tabular}{|c|c|c|}
\hline & PM & FM (small delay) \\
\hline \hline Gain & $\left|Z_{\text {in }}\right|\left|Z_{\text {out }}\right| 4\left(\frac{i_{d c} \pi}{V_{\pi}}\left|\sin \left(\pi f_{1} \tau\right)\right|\right)^{2}$ & $\frac{\left|Z_{\text {out }}\right|}{\left|Z_{\text {in }}\right|} 4\left(\pi \eta i_{d c} \tau\right)^{2}$ \\
\hline Shot noise NF & $1+\frac{q V_{\pi}^{2}}{\left|Z_{\text {in }}\right| 2 i_{d c} \pi^{2} k_{B} T_{K}\left|\sin \left(\pi f_{1} \tau\right)\right|^{2}}$ & $1+\frac{\Delta Z_{\text {in }} \mid q}{\Re P_{\text {opt }} \eta^{2} 2 \pi^{4} \tau^{2} k_{B} T_{K}}$ \\
\hline Phase noise NF & $1+\frac{\Delta \nu V_{\pi}^{2}}{\left|Z_{\text {in }}\right| \pi^{3} f_{1}^{2} k_{B} T_{K}}$ & $1+\frac{\left|Z_{\text {in }}\right| \Delta \nu}{\eta^{2} \pi k_{B} T_{K}}$ \\
\hline OIP3 & $4\left|Z_{\text {out }}\right| i_{d c}^{2} \frac{\sin \left(\pi f_{1} \tau\right)}{\sin \left(\pi f_{2} \tau\right)} \mid$ & $4\left|Z_{\text {out }}\right| i_{d c}^{2}$ \\
\hline Shot noise $S F D R_{3}$ & $\left(\frac{\sin ^{4}\left(\pi f_{1} \tau\right) 2 i_{d c}}{\left|\sin \left(\pi f_{2} \tau\right)\right| q B}\right)^{2 / 3}$ & $\pi^{2} f_{1}^{2} \tau^{2}\left(\frac{2 i_{d c}}{q B}\right)^{2 / 3}$ \\
\hline Phase noise $S F D R_{3}$ & $\left(\frac{f_{1}^{2} \pi}{\Delta \nu B\left|\sin \left(\pi f_{1} \tau\right) \sin \left(\pi f_{2} \tau\right)\right|}\right)^{2 / 3}$ & $\left(\frac{1}{\Delta \nu B \pi \tau^{2}}\right)^{2 / 3}$ \\
\hline
\end{tabular}

where $A$ is a slope in units of inverse frequency, $f_{b}$ is a bias frequency offset from the carrier, and $\tau$ is a time delay giving the filter linear phase. The intensity response is

$$
h_{n, p} h_{n, p}^{*}=A\left(f_{b}+n f_{1}+p f_{2}\right)
$$

which is linear in slope $A$. Using the transfer function, I evaluate the link constants:

$$
\begin{aligned}
& X_{0}=\sqrt{A f_{b}} e^{-j 2 \pi f_{b} \tau} \\
& X_{1}=A f_{b}\left(\sqrt{1+\frac{f_{1}}{f_{b}}}-\sqrt{1-\frac{f_{1}}{f_{b}}}\right) e^{-j 2 \pi f_{1} \tau} \\
& X_{2}=A f_{b}\left(\sqrt{1+2 \frac{f_{1}}{f_{b}}}-\sqrt{1-\frac{f_{1}}{f_{b}}}\right. \\
& \left.-2 \sqrt{1+\frac{f_{1}}{f_{b}}} \sqrt{1-\frac{f_{1}}{f_{b}}}\right) e^{-j 4 \pi f_{1} \tau} \\
& X_{3}=A f_{b}\left(2 \sqrt{1+\frac{f_{1}}{f_{b}}-\frac{f_{2}}{f_{b}}} \sqrt{1-\frac{f_{1}}{f_{b}}}\right. \\
& -2 \sqrt{1-\frac{f_{1}}{f_{b}}+\frac{f_{2}}{f_{b}}} \sqrt{1+\frac{f_{1}}{f_{b}}} \sqrt{1-2 \frac{f_{1}}{f_{b}}+\frac{f_{2}}{f_{b}}} \\
& -\sqrt{1+2 \frac{f_{1}}{f_{b}}-\frac{f_{2}}{f_{b}}}+\sqrt{1+2 \frac{f_{1}}{f_{b}}} \sqrt{1+\frac{f_{2}}{f_{b}}} \\
& \left.-\sqrt{1-2 \frac{f_{1}}{f_{b}}} \sqrt{1-\frac{f_{2}}{f_{b}}}\right) e^{-j 2 \pi \tau\left(2 f_{1}-f_{2}\right)}
\end{aligned}
$$

Generally, $X_{2}$ and $X_{3}$ are non-zero for this discriminator, even if the square roots are expanded. This means that a discriminator that is linear in optical intensity will still produce second-order and third-order distortion. Mixing in the photodetector 
produces cross terms that are not eliminated. An FM discriminator that is linear is optical intensity will not produce a distortion-less link.

The ideal discriminator for the link is a pair of optical filters that are linear in electric field. Within one period, the field transmission ramps linearly with frequency, and the filter has linear phase. The transfer functions near the carrier are

$$
\begin{aligned}
& h_{n, p}^{1}=\frac{1}{\sqrt{2}} A\left(f_{b}+n f_{1}+p f_{2}\right) \exp \left[-j 2 \pi\left(f_{b}+n f_{1}+p f_{2}\right) \tau\right] \\
& h_{n, p}^{2}=\frac{1}{\sqrt{2}} A\left(f_{b}-n f_{1}-p f_{2}\right) \exp \left[-j 2 \pi\left(f_{b}+n f_{1}+p f_{2}\right) \tau\right]
\end{aligned}
$$

where $A$ is a slope in units of inverse frequency and $\tau$ is a time delay. The $1 / \sqrt{2}$ prefactor is an optical splitter before two physical filters. I define the constant $T$ to describe the dc bias of the filter, which is the fraction of optical power transmitted by the filter at the optical carrier frequency. The link distortion constants are

$$
\begin{aligned}
& X_{0}^{1}=A^{2} f_{b}^{2} / 2 \equiv T / 2 \\
& X_{1}^{1}=A f_{1} T^{1 / 2} e^{-j 2 \pi f_{1} \tau} \\
& Y_{1}^{1}=T e^{-j 2 \pi f_{1} \tau} \\
& X_{2}^{1}=A^{2} f_{1}^{2} e^{-j 4 \pi f_{1} \tau} \\
& X_{3}^{1}=0
\end{aligned}
$$

and

$$
\begin{aligned}
& X_{0}^{2}=T / 2 \\
& X_{1}^{2}=-A f_{1} T^{1 / 2} e^{-j 2 \pi f_{1} \tau} \\
& Y_{1}^{2}=T e^{-j 2 \pi f_{1} \tau} \\
& X_{2}^{2}=A^{2} f_{1}^{2} e^{-j 4 \pi f_{1} \tau} \\
& X_{3}^{2}=0
\end{aligned}
$$

All higher order link-constants are zero. The non-zero values of $X_{2}^{z}$ are due to the squaring of the signal at the detector. The distortion is caused by the first-order sidebands beating with each other. However, because the second harmonics are in phase, they cancel at the balanced detector, giving perfect distortionless performance. The current component at the fundamental frequency will be $180^{\circ}$ out of phase between the two photodetectors, but the second-harmonic will be in phase. After the balanced detector, the only term that does not cancel is

$$
\left|X_{1}\right|=2 A f_{1} T^{1 / 2}
$$

It is important to note that the intensity modulation term also cancels because of balanced detection. Residual intensity modulation of the laser and relative intensity noise present before the demodulation will not be present at the output of this system.

In the small modulation depth approximation, this ideal link has no other higherorder distortion. Using a symbolic algebra solver, I verified that the current is zero for 
all intermodulation and harmonic frequencies up to sixth order. At a given harmonic, sum or difference frequency, if all the sidebands in the sum in (2.9) corresponding to that frequency fall within a region of the filter that closely approximates the desired linear ramp function, the output current is zero.

Additional sources of nonlinearity are the frequency modulated laser source, optical fibers and photodetector. For sufficient modulation depth, the dominant sidebands will fall outside the bandwidth of the filter and this saturation will cause nonlinearities.

\subsubsection{Noise and gain}

In this section, I will consider the effect of the bias, $\mathrm{T}$, on the noise figure of the link. Low biasing the filter, to decrease the dc current at the detector, had been suggested by [54] and others to improve the noise figure (NF) of a PM or FM link. However, there is a tradeoff between decreasing the dc, which decreases shot noise, and reducing the signal gain, so an optimal bias point must be found. The filter cannot be biased exactly at the null or the link would have zero output current, since I find in (2.64) that the output is proportional to the square root of the bias. This is consistent with experience with carrier suppression on IM-DD links.

The noise figure of the link is comprised of a term for an attenuated link, the shot noise component, and the phase noise component. Intensity noise does not appear because it is canceled with the balanced detection. The noise figures for PM and FM are given by as follows. They are written in terms of the dc photocurrent at the detectors, instead of the total optical power before the filters, since current handling of the diodes is usually a limiting factor.

$$
\begin{aligned}
N F_{P M}=1 & +\frac{T V_{\pi}^{2}}{\left|Z_{\text {in }}\right|\left|Z_{\text {out }}\right| 4 \pi^{2} i_{d c}^{2} A^{2} f_{1}^{2}} \\
& +\frac{q V_{\pi}^{2} T}{\left|Z_{\text {in }}\right| i_{d c} \pi^{2} 2 k_{B} T_{K} A^{2} f_{1}^{2}}+\frac{\triangle \nu V_{\pi}^{2}}{\left|Z_{\text {in }}\right| \pi^{3} f_{1}^{2} k_{B} T_{K}} \\
N F_{F M}=1 & +\frac{\left|Z_{\text {in }}\right| T}{\left|Z_{\text {out }}\right| 4 \eta^{2} i_{d c}^{2} A^{2}} \\
& +\frac{\left|Z_{\text {in }}\right| q T}{i_{\text {dc }} \eta^{2} 2 k_{B} T_{K} A^{2}}+\frac{\left|Z_{\text {in }}\right| \triangle \nu}{\eta^{2} \pi k_{B} T_{K}}
\end{aligned}
$$

A useful question is whether it makes sense to low bias the filter in an attempt to improve the noise figure. The answer depends on whether the designer is limited by optical power available or by the maximum photocurrent the photodetectors can handle. For a fixed current, for which the optical power is increased to maintain, the derivative of the $\mathrm{NF}$ with respect to the bias is

$$
\begin{aligned}
& \frac{\partial N F_{P M}}{\partial T}=\frac{V_{\pi}^{2}}{\left|Z_{\text {in }}\right|\left|Z_{\text {out }}\right| 4 \pi^{2} i_{d c}^{2} A^{2} f_{1}^{2}}+\frac{q V_{\pi}^{2}}{\left|Z_{\text {in }}\right| i_{d c} \pi^{2} 2 k_{B} T_{K} A^{2} f_{1}^{2}} \\
& \frac{\partial N F_{F M}}{\partial T}=\frac{\left|Z_{\text {in }}\right|}{\left|Z_{\text {out }}\right| 4 \eta^{2} i_{d c}^{2} A^{2}}+\frac{\left|Z_{\text {in }}\right| q}{i_{d c} \eta^{2} 2 k_{B} T_{K} A^{2}}
\end{aligned}
$$


Table 2.4: Gain and noise figure expressions for the complementary linear-field demodulated PM-DD link.

\begin{tabular}{|c|c|c|}
\hline & PM & FM \\
\hline \hline Gain & $\left|Z_{\text {in }}\right|\left|Z_{\text {out }}\right| 4 T^{-1}\left(\frac{\pi i_{d c}}{V_{\pi}} A f_{1}\right)^{2}$ & $\frac{\left|Z_{\text {out }}\right|}{\left|Z_{\text {in }}\right|} 4 T^{-1}\left(\eta i_{d c} A\right)^{2}$ \\
\hline Shot noise NF & $1+\frac{q V_{\pi}^{2}}{\left|Z_{\text {in }}\right| \Re P_{o p t} \pi^{2} 2 k_{B} T_{K} A^{2} f_{1}^{2}}$ & $1+\frac{\left|Z_{\text {in }}\right| q}{\Re P_{\text {opt }} \eta^{2} 2 k_{B} T_{K} A^{2}}$ \\
\hline Phase noise NF & $1+\frac{\triangle \nu V_{\pi}^{2}}{\left|Z_{\text {in }}\right| \pi^{3} f_{1}^{2} k_{B} T_{K}}$ & $1+\frac{\left|Z_{\text {in }}\right| \Delta \nu}{\eta^{2} \pi k_{B} T_{K}}$ \\
\hline Laser linewidth limit & $\left(N F_{p n}-1\right) \frac{\left|Z_{i n}\right| \pi^{3} f_{1}^{2} k_{B} T_{K}}{V_{\pi}^{2}}$ & $\left(N F_{p n}-1\right) \frac{\eta^{2} k_{B} T_{K} \pi}{\left|Z_{\text {in }}\right|}$ \\
\hline
\end{tabular}

These are always positive quantities so the noise figure monotonically decreases with decreasing bias as long as the current is maintained. However, the phase noise will begin to dominate over the shot noise when $T q<2 i_{d c} A^{2} \frac{\Delta \nu}{\pi}$, and any NF improvement will be negligible. For example, with $\triangle \nu=1 \mathrm{MHz}$ and $A=1 / 50 \mathrm{GHz}$, choosing a low bias point only makes sense if the maximum dc current is less than $160 \mu \mathrm{A}$.

If the available optical power is fixed, $i_{d c}=\Re P_{o p t} T$, then the derivative of the NF with respect to the bias is always negative:

$$
\begin{aligned}
& \frac{\partial N F_{P M}}{\partial T}=-\frac{V_{\pi}^{2}}{\left|Z_{\text {in }}\right|\left|Z_{\text {out }}\right| 4 \pi^{2} \Re^{2} P_{\text {opt }}^{2} T^{2} A^{2} f_{1}^{2}} \\
& \frac{\partial N F_{F M}}{\partial T}=-\frac{\left|Z_{\text {in }}\right|}{\left|Z_{\text {out }}\right| 4 \eta^{2} \Re^{2} P_{\text {opt }}^{2} T^{2} A^{2}}
\end{aligned}
$$

Reducing the bias only serves to reduce the gain of the link and the NF gets worse with the lower bias.

For high optical powers, the NF is phase noise limited. This is independent of the filter bias and the slope of the filter. Because random frequency fluctuations are added to the optical carrier at the same time as it is modulated, the maximally achievable SNR is set at the laser, and cannot be improved by the rest of the system. This formula sets a fundamental relationship between the maximally achievable noise figure, the laser linewidth and the modulation efficiency. For a given noise figure and modulation efficiency, the maximum laser linewidth is given in the table above. This fundamental relationship between modulation efficiency, linewidth and noise figure is plotted in Fig. 2.5 for a range of typical values.

\subsubsection{Transfer function curvature}

The realized complementary, linear-field filters will be non-ideal in their phase and amplitude transfer functions. It is useful to define a mask or envelope for the filter transfer function's phase and amplitude which will guarantee a desired nonlinearity performance. I find that a mask with the form of a quadratic equation of frequency offset from the carrier will give a tractable bound for OIP2, and a cubic mask will give a tractable bound for OIP3. Local derivatives do not affect distortion as long as the function falls within given envelope. 
Figure 2.5: Phase noise limited noise figure versus linewidth and modulation efficiency, assuming a 50 ohm impedance.

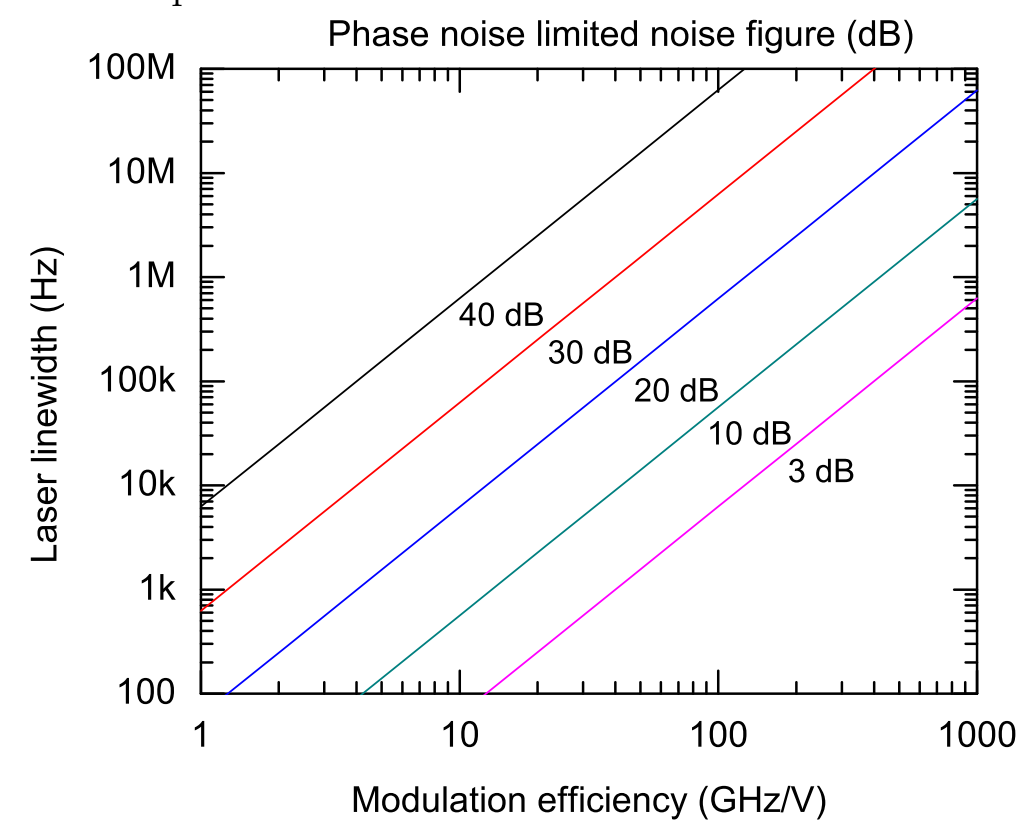

The realized transfer function for one branch of the discriminator is written in the form

$$
h(f)=\frac{1}{\sqrt{2}}\left(\sqrt{T}+A f+\triangle_{a}(f)\right) \exp \left[-j 2 \pi f \tau-j \triangle_{p}(f)\right]
$$

where $\triangle_{a}(f)$ and $\triangle_{p}(f)$ are the deviations from the ideal phase and amplitude, and $f$ is the offset from the carrier.

Figure 2.6 on page 33 illustrates the masks for the amplitude and phase for bounding the second-order figures of merit. The deviations from ideal for the amplitude and phase must fall within bounds which relax further away from the carrier frequency:

$$
\begin{aligned}
& \triangle_{a}(f)=\varepsilon_{2}(f) A^{2} f^{2}, \\
& \left|\varepsilon_{2}(f)\right| \leq e_{2, \max }
\end{aligned}
$$

and

$$
\begin{aligned}
\triangle_{p}(f) & =\phi_{2} A^{2} f^{2} \\
\left|\phi_{2}(f)\right| & \leq \phi_{2, \max }
\end{aligned}
$$

where $\varepsilon_{2, \max }$ and $\phi_{2, \max }$ are small positive constants. For a two-tone test derivation, I make the approximations that the modulation tones are closely spaced, $f_{1} \approx f_{2} \equiv f$, the phase deviation is small so that $\exp [-j \phi] \approx 1-j \phi$ and the frequency is low with respect to the bias so that $A f \leq \sqrt{T}$. For the OIP2 derivation, I use the second harmonic as the distortion term of interest. I also assume complementary filters and 
Figure 2.6: Illustration of the quadratic envelope on the transfer function that bounds the second-order figures of merit for the complementary linear-field discriminator.

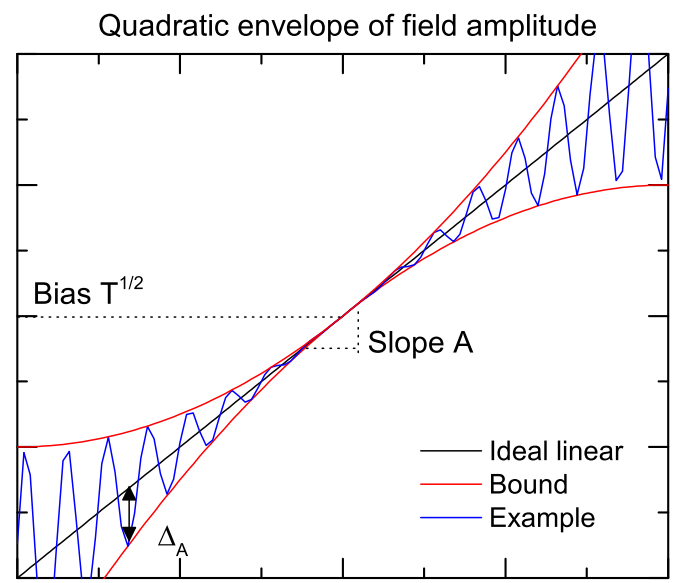

Offset frequency from carrier $(\mathrm{Hz})$

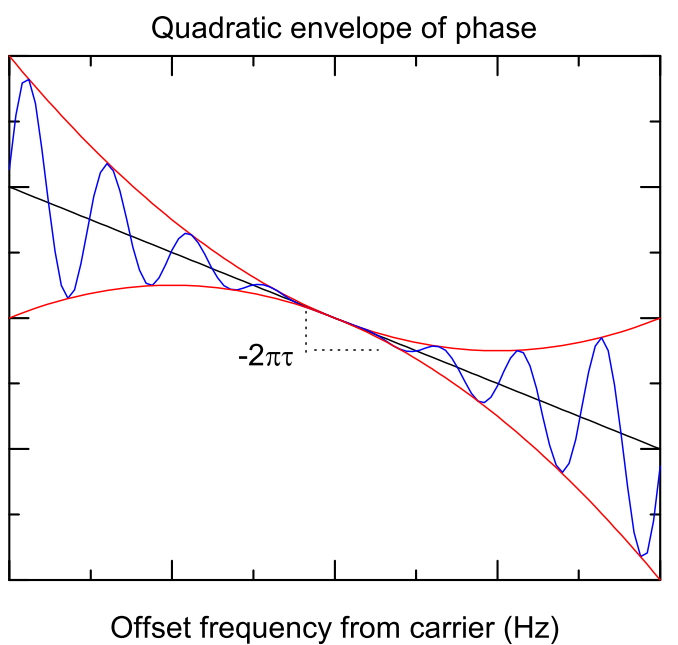

balanced detection. The two tone transfer function for one branch is

$$
\begin{aligned}
h_{n, p}^{1}= & \frac{1}{\sqrt{2}}\left(\sqrt{T}+A\left(n f_{1}+p f_{2}\right)+\varepsilon_{2}\left(n f_{1}+p f_{2}\right) A^{2}\left(n f_{1}+p f_{2}\right)^{2}\right) \\
& \cdot \exp \left[-j 2 \pi\left(n f_{1}+p f_{2}\right) \tau-j \phi_{2}\left(n f_{1}+p f_{2}\right) A^{2}\left(n f_{1}+p f_{2}\right)^{2}\right]
\end{aligned}
$$

After algebraic simplifications, assuming the worst case addition of errors, the second-order link distortion constant is bounded by

$$
\left|X_{2}\right| \leq A^{2} f^{2}\left(C_{1} \varepsilon_{2, \max }+j C_{2} \phi_{2, \max }\right)
$$

where

$$
\begin{aligned}
& C_{1}=12 \sqrt{T} \\
& C_{2}=12 T-4 A^{2} f^{2}
\end{aligned}
$$

and its magnitude is therefore

$$
\left|X_{2}\right| \leq A^{2} f^{2} 12 \sqrt{\varepsilon_{2, \max }^{2} T+\phi_{2, \max }^{2}\left(T-\frac{1}{3} A^{2} f^{2}\right)^{2}} .
$$

The second-order output intercept point is lower bounded as

$$
\text { OIP2 } \geq \frac{8}{9} R_{\text {load }} i_{d c}^{2} \frac{1}{\varepsilon_{2, \text { max }}^{2} T+\phi_{2, \text { max }}^{2}\left(T-\frac{1}{3} A^{2} f^{2}\right)^{2}}
$$

for the worst case frequency,

$$
\text { OIP2 } \geq \frac{8}{9} R_{\text {load }} i_{d c}^{2} \frac{1}{\varepsilon_{2, \max }^{2} T+\phi_{2, \text { max }}^{2} T^{2}}
$$


Figure 2.7: Illustration of the cubic envelope on the transfer function that bounds the third-order figures of merit for the complementary linear-field discriminator.

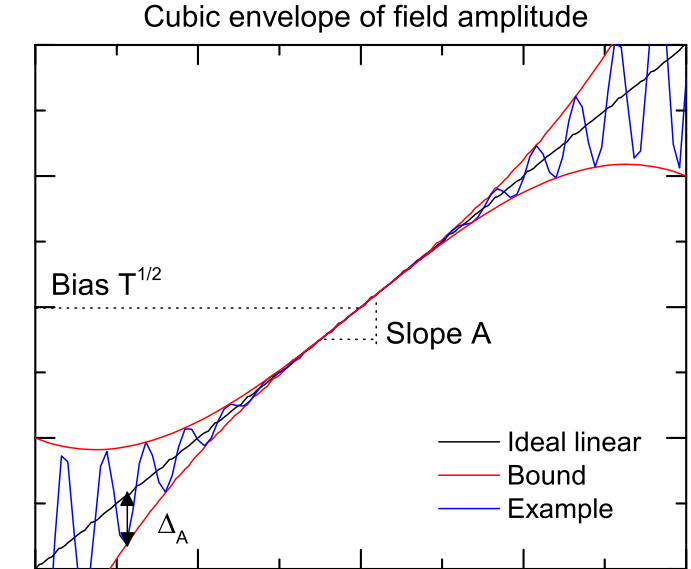

Offset frequency from carrier $(\mathrm{Hz})$

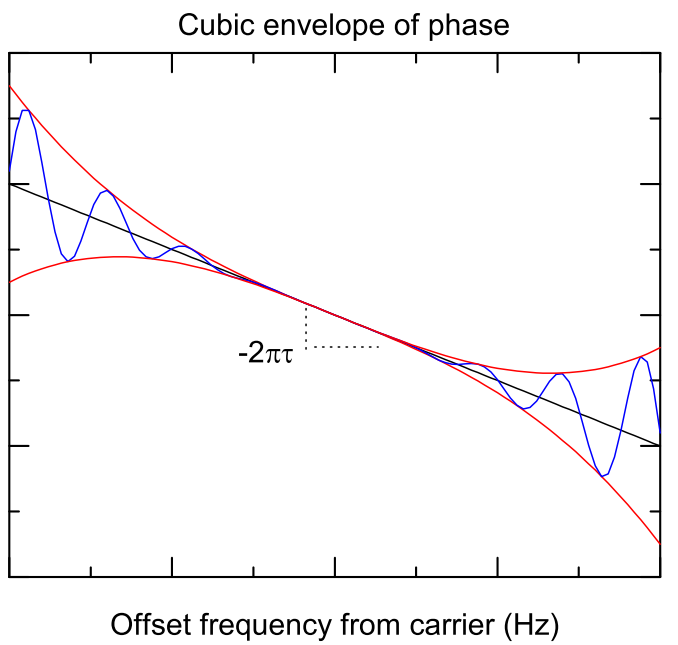

and for the important half field-bias case where $\sqrt{T}=1 / 2$,

$$
\text { OIP2 } \geq \frac{32}{9} R_{\text {load }} i_{d c}^{2} \frac{1}{\varepsilon_{2, \text { max }}^{2}+\frac{1}{4} \phi_{2, \text { max }}^{2}}
$$

Figure 2.7 on page 34 illustrates the masks for the amplitude and phase for bounding the third-order figures of merit. The deviations from ideal for the amplitude and phase must fall within bounds which relax further away from the carrier frequency:

$$
\begin{aligned}
& \triangle_{a}(f)=\varepsilon_{3}(f) A^{3} f^{3}, \\
& \left|\varepsilon_{3}(f)\right| \leq e_{3, \max }
\end{aligned}
$$

and

$$
\begin{aligned}
\triangle_{p}(f) & =\phi_{3}(f) A^{3} f^{3} \\
\left|\phi_{3}(f)\right| & \leq \phi_{3, \max }
\end{aligned}
$$

where $\varepsilon_{3, \max }$ and $\phi_{3, \max }$ are small positive constants. For a two-tone test derivation, I make the same approximations as before. The two tone transfer function for one branch is

$$
\begin{aligned}
h_{n, p}^{1}= & \frac{1}{\sqrt{2}}\left(\sqrt{T}+A\left(n f_{1}+p f_{2}\right)+\varepsilon_{3}\left(n f_{1}+p f_{2}\right) A^{3}\left(n f_{1}+p f_{2}\right)^{3}\right) \\
& \cdot \exp \left[-j 2 \pi\left(n f_{1}+p f_{2}\right) \tau-j \phi_{3}\left(n f_{1}+p f_{2}\right) A^{3}\left(n f_{1}+p f_{2}\right)^{3}\right]
\end{aligned}
$$

After algebraic simplifications, assuming the worst case addition of errors, the third-order link distortion constant, given by is bounded by 


$$
X_{3} \leq A^{3} f^{3}\left(C_{1} \varepsilon_{3, \max }+j C_{2} \phi_{3, \max }\right)
$$

where

$$
\begin{aligned}
& C_{1}=24 \sqrt{T} \\
& C_{2}=24 T+36 A^{2} f^{2}
\end{aligned}
$$

and its magnitude is therefore

$$
\left|X_{3}\right| \leq A^{3} f^{3} 24 T^{1 / 2} \sqrt{\varepsilon_{3, \max }^{2}+\phi_{3, \max }^{2} T\left(1+\frac{3}{2} A^{2} f^{2} / T\right)^{2}} .
$$

The third-order output intercept point is lower bounded as

$$
\text { OIP3 } \geq \frac{4}{3} R_{\text {load }} \frac{i_{d c}^{2}}{T} \frac{1}{\sqrt{\varepsilon_{3, \text { max }}^{2}+\phi_{3, \text { max }}^{2} T\left(1+\frac{3}{2} A^{2} f^{2} / T\right)^{2}}}
$$

for the worst case frequency,

$$
\text { OIP3 } \geq \frac{4}{3} R_{\text {load }} \frac{i_{d c}^{2}}{T} \frac{1}{\sqrt{\varepsilon_{3, \text { max }}^{2}+\phi_{3, \text { max }}^{2} T(1+3 / 32 T)^{2}}}
$$

and for the important half field-bias case where $\sqrt{T}=1 / 2$,

$$
\text { OIP3 } \geq \frac{16}{3} R_{\text {load }} i_{d c}^{2} \frac{1}{\sqrt{\varepsilon_{3, \max }^{2}+\phi_{3, \max }^{2} \frac{121}{256}}}
$$

I performed Monte Carlo simulations to verify these error bounds. I created a complementary linear-field transfer function and added random deviations that fall within the mask. The transfer function was used to analytically calculate the distortion figures of merit. This was repeated 1000 times for each parameter, and the worst case was saved. The worst-case simulated distortions fell within 0.5 to 2 $\mathrm{dB}$ above the lower bound, making this a suitable mask. The best cases sometimes outperformed the bound by 10s of decibels, but this was highly dependent on the modulation frequency.

\subsubsection{Residual intensity modulation}

Residual intensity modulation sets a lower limit on the distortion for a link using complementary linear field discriminators. The effect of residual IM can be obtained from (2.9). It is difficult to write a general expression, but it is possible to expand some individual terms. In lowest polynomial order of the modulation depth, the 
Table 2.5: Expressions for the worst case OIP2, OIP3, and spurious free dynamic range for complimentary linear-field demodulation limited by filter curvature.

\begin{tabular}{|c|c|c|}
\hline & $\overline{\mathrm{PM}}$ & FM \\
\hline$O I P 2$ & $\frac{8}{9}\left|Z_{\text {out }}\right| \frac{i_{d c}^{2}}{T} \frac{1}{\varepsilon_{2, \max }^{2}+\phi_{2, \max }^{2} T}$ & $\frac{8}{9}\left|Z_{\text {out }}\right| \frac{i_{d c}^{2}}{T} \frac{1}{\varepsilon_{2, \max }^{2}+\phi_{2, \max }^{2} T}$ \\
\hline Shot noise $S F D R_{2}$ & $\frac{2}{3} \sqrt{\frac{\Re P_{o p t}}{q B\left(\varepsilon_{2, \max }^{2}+\phi_{2, \max }^{2} T\right)}}$ & $\frac{2}{3} \sqrt{\frac{\Re P_{o p t}}{q B\left(\varepsilon_{2, \text { max }}^{2}+\phi_{2, \text { max }}^{2} T\right)}}$ \\
\hline Phase noise $S F D R_{2}$ & $\frac{1}{3 A} \sqrt{\frac{2 \pi}{\Delta \nu B\left(\varepsilon_{2, \max }^{2}+\phi_{2, \max }^{2} T\right)}}$ & $\frac{1}{3 A} \sqrt{\frac{2 \pi}{\Delta \nu B\left(\varepsilon_{2, \max }^{2}+\phi_{2, \max }^{2} T\right)}}$ \\
\hline OIP3 & $\frac{4}{3}\left|Z_{\text {out }}\right| \frac{i_{d c}^{2}}{T} \frac{1}{\sqrt{\varepsilon_{3, \max }^{2}+\phi_{3, \max }^{2} T}}$ & $\frac{4}{3}\left|Z_{\text {out }}\right| \frac{i_{d c}^{2}}{T} \frac{1}{\sqrt{\varepsilon_{3, \max }^{2}+\phi_{3, \max }^{2} T}} \frac{f_{2}}{f_{1}}$ \\
\hline Shot noise $S F D R_{3}$ & $\left(\frac{2 \Re P_{o p t}}{3 q B \sqrt{\varepsilon_{3, \text { max }}^{2}+\phi_{3, \text { max }}^{2} T}}\right)^{2}$ & $\left(\frac{2 \Re P_{\text {opt }}}{3 q B \sqrt{\varepsilon_{3, \text { max }}^{2}+\phi_{3, \text { max }}^{2} T}} \frac{f_{2}}{f_{1}}\right)$ \\
\hline Phase noise $\mathrm{SFDR}_{3}$ & $\left(\frac{\pi}{3 A^{2} \Delta \nu B \sqrt{\varepsilon_{3, \max }^{2}+\phi_{3, \max }^{2} T}}\right)^{2 / 3}$ & $\left(\frac{\pi}{3 A^{2} \Delta \nu B \sqrt{\varepsilon_{3, \text { max }}^{2}+\phi_{3, \text { max }}^{2} T}} \frac{f_{2}}{f_{1}}\right)$ \\
\hline
\end{tabular}

Figure 2.8: Monte Carlo simulation results to test the suitability of the derived bounds on the OIP2 and OIP3. Each point is the worst case of 1000 trials with random errors, and is compared to the analytical bounds. We assume closely spaced tones around 2 $\mathrm{GHz}, 1 / 10 \mathrm{GHz}$ slope, $5 \mathrm{~mA}$ of current per detector $\left(i_{d c}=10 \mathrm{~mA}\right), 50 \mathrm{ohm}$ impedance, and 0.5 amplitude bias, $T=0.25$. The analytical expression bounds the simulation within less than $2 \mathrm{~dB}$.
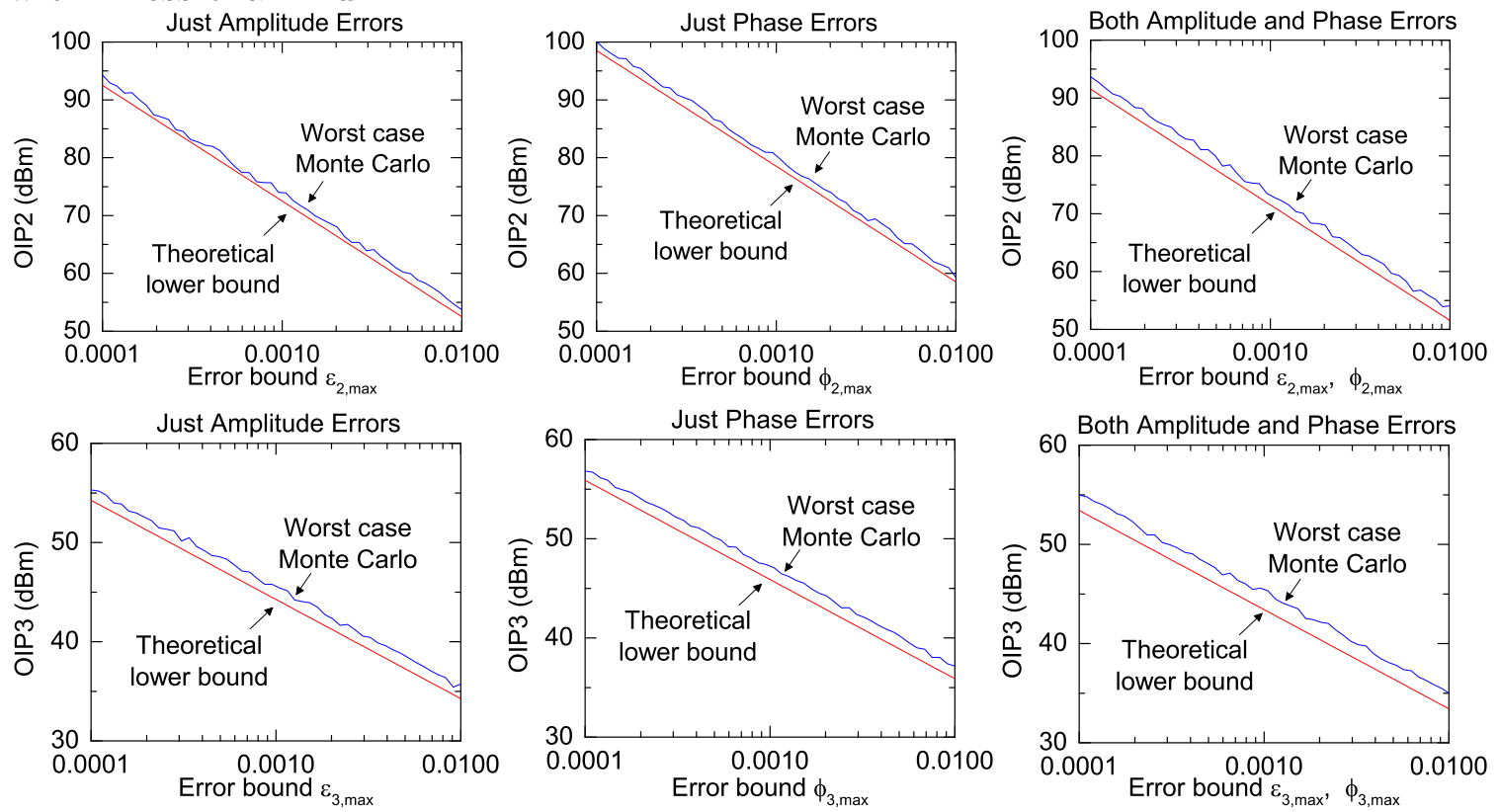
currents of interest are

$$
\begin{aligned}
& i_{d c}^{z} \approx \Re P_{o p t}\left|h_{0,0}^{z}\right|^{2} \\
& i_{f_{1}}^{z} \approx \Re P_{o p t} R e\left\{\left[\beta_{1}\left(h_{1,0}^{z} h_{0,0}^{z *}-h_{0,0}^{z} h_{-1,0}^{z *}\right)\right.\right. \\
& \left.\left.+\frac{1}{2} m_{1}\left(h_{1,0}^{z} h_{0,0}^{z *}+h_{0,0}^{z} h_{-1,0}^{z *}\right) e^{j \phi}\right] \exp \left[j 2 \pi f_{1} t\right]\right\} \\
& i_{2 f_{1}}^{z} \approx \Re P_{\text {opt }} \frac{1}{4} R e\left\{\left[\beta_{1}^{2}\left(h_{2,0}^{z} h_{0,0}^{z *}-2 h_{1,0}^{z} h_{-1,0}^{z *}+h_{0,0}^{z} h_{-2,0}^{z *}\right)\right.\right. \\
& \left.+m_{1} \beta_{1}\left(h_{2,0}^{z} h_{0,0}^{z *}-h_{0,0}^{z} h_{-2,0}^{z *}\right) e^{j \phi}+\frac{1}{2} m_{1}^{2} h_{1,0}^{z} h_{-1,0}^{z *} e^{j 2 \phi}\right] \\
& \left.\exp \left[j 4 \pi f_{1} t\right]\right\} \\
& i_{2 f_{1}-f_{2}}^{z} \approx \Re P_{o p t} \frac{1}{8} R e\left\{\left[\beta _ { 1 } ^ { 2 } \beta _ { 2 } \left(-h_{2,-1}^{z} h_{0,0}^{z *}+h_{2,0}^{z} h_{0,1}^{z *}+2 h_{1,-1}^{z} h_{-1,0}^{z *}\right.\right.\right. \\
& \left.-2 h_{1,0}^{z} h_{-1,1}^{z *}-h_{0,-1}^{z} h_{-2,0}^{z *}+h_{0,0}^{z} h_{-2,1}^{z *}\right) \\
& +m_{1} \beta_{1} \beta_{2} e^{j \phi}\left(h_{2,0}^{z} h_{0,1}^{z *}-h_{2,-1}^{z} h_{0,0}^{z *}+h_{0,-1}^{z} h_{-2,0}^{z *}-h_{0,0}^{z} h_{-2,1}^{z *}\right) \\
& +\frac{1}{2} m_{2} \beta_{1}^{2} e^{-j \phi}\left(h_{2,0}^{z} h_{0,1}^{z *}+h_{2,-1}^{z} h_{0,0}^{z *}-2 h_{1,0}^{z} h_{-1,1}^{z *}\right. \\
& \left.-2 h_{1,-1}^{z} h_{-1,0}^{z *}+h_{0,0}^{z} h_{-2,1}^{z *}+h_{0,-1}^{z} h_{-2,0}^{z *}\right) \\
& +\frac{1}{2} m_{1}^{2} \beta_{2} e^{j 2 \phi}\left(-h_{1,-1}^{z} h_{-1,0}^{z *}+h_{1,0}^{z} h_{-1,1}^{z *}\right) \\
& +\frac{1}{2} m_{1} m_{2} \beta_{1}\left(h_{2,0}^{z} h_{0,1}^{z *}+h_{1,-1}^{z} h_{-1,0}^{z *}-h_{1,0}^{z} h_{-1,1}^{z *}-h_{0,-1}^{z} h_{-2,0}^{z *}\right) \\
& \left.\exp \left[j 2 \pi\left(2 f_{1}-f_{2}\right) t\right]\right\}
\end{aligned}
$$

It is useful to normalize the IM to the FM. One method of normalization is to look at the optical power the IM and angle modulation contribute to the first-order optical sidebands in the small signal approximation. The optical power in the first order sidebands from the IM is $P_{\text {opt }} m_{1}^{2} / 16$. The optical power in the first order sidebands due to the PM or FM is $P_{o p t} \beta^{2} / 4$. I define a relative residual IM, $\Gamma$, as

$$
\Gamma \equiv \frac{m}{2 \beta}
$$

The corrected expressions for the link distortion constants, including the effect of 
residual intensity modulation, are

$$
\begin{aligned}
X_{0}^{z}= & h_{0,0}^{z} h_{0,0}^{z *} \\
X_{1}^{z}= & h_{1,0}^{z} h_{0,0}^{z *}-h_{0,0}^{z} h_{-1,0}^{z *} \\
& +\Gamma\left(h_{1,0}^{z} h_{0,0}^{z *}+h_{0,0}^{z} h_{-1,0}^{z *}\right) e^{j \phi} \\
X_{2}^{z}= & h_{2,0}^{z} h_{0,0}^{z *}-2 h_{1,0}^{z} h_{-1,0}^{z *}+h_{0,0}^{z} h_{-2,0}^{z *} \\
& +2 \Gamma\left(h_{2,0}^{z} h_{0,0}^{z *}-h_{0,0}^{z} h_{-2,0}^{z *}\right) e^{j \phi} \\
& +2 \Gamma^{2} h_{1,0}^{z} h_{-1,0}^{z *} e^{j 2 \phi} \\
X_{3}^{z}= & -h_{2,-1}^{z} h_{0,0}^{z *}+h_{2,0}^{z} h_{0,1}^{z *}+2 h_{1,-1}^{z} h_{-1,0}^{z *} \\
& +h_{0,0}^{z} h_{-2,1}^{z *}-h_{0,-1}^{z} h_{-2,0}^{z *}-2 h_{1,0}^{z} h_{-1,1}^{z *} \\
& +2 \Gamma e^{j \phi}\left(h_{2,0}^{z} h_{0,1}^{z *}-h_{2,-1}^{z} h_{0,0}^{z *}+h_{0,-1}^{z} h_{-2,0}^{z *}-h_{0,0}^{z} h_{-2,1}^{z *}\right) \\
& +\Gamma e^{-j \phi}\left(h_{2,0}^{z} h_{0,1}^{z *}+h_{2,-1}^{z} h_{0,0}^{z *}-2 h_{1,0}^{z} h_{-1,1}^{z *}\right. \\
& \left.-2 h_{1,-1}^{z} h_{-1,0}^{z *}+h_{0,0}^{z} h_{-2,1}^{z *}+h_{0,-1}^{z} h_{-2,0}^{z *}\right) \\
& +2 \Gamma^{2} e^{j 2 \phi}\left(-h_{1,-1}^{z} h_{-1,0}^{z *}+h_{1,0}^{z} h_{-1,1}^{z *}\right) \\
& +2 \Gamma^{2}\left(h_{2,0}^{z} h_{0,1}^{z *}+h_{1,-1}^{z} h_{-1,0}^{z *}-h_{1,0}^{z} h_{-1,1}^{z *}-h_{0,-1}^{z} h_{-2,0}^{z *}\right)
\end{aligned}
$$

For the complementary, linear-field demodulation, the magnitude of the distortion constants are

$$
\begin{aligned}
& \left|X_{1}\right|=2 A f_{1} T^{1 / 2} \\
& \left|X_{2}\right|=8 A f_{1} T^{1 / 2} \Gamma|\cos (\phi / 2)| \\
& \left|X_{3}\right|=4 A \Gamma^{2} T^{1 / 2}\left|2 f_{1}+f_{2} \exp [j 2 \phi]\right|
\end{aligned}
$$

Since the intensity modulation is residual, the frequency modulation will be much greater than the intensity modulation. With balanced detection, both the dominant second-harmonic terms and dominant IMD3 terms are quadratic with the intensity modulation are linear in the IM. The values for residual intensity modulation limited OIP2 and OIP3 are in the below table. A set of example curves are shown in 2.9. It is interesting to note that the values for the spurious free dynamic range are independent of the bias.

\subsubsection{Dispersion}

The dispersion of the optical fiber also increases the distortion of a PM-DD or FMDD link. The dispersion is modeled by multiplying the filter transfer function by the term $\exp \left[-j \pi D z\left(n f_{1}+p f_{2}\right)^{2}\right]$, where $D$ is the fiber dispersion parameter and $z$ is the fiber length. The figure below, 2.10, shows example curves of the upper limit the dispersion sets on OIP3. It degrades by $20 \mathrm{~dB}$ per decade of fiber length. This can be corrected by using a length of dispersion compensated fiber, or by designing a discriminator filter's transfer function to include the inverse of the dispersion. The mechanism for the dispersion's impact on the link distortion is conversion of phase or frequency modulation to intensity modulation. 
Table 2.6: Expressions for OIP2 and OIP3 for complimentary linear-field demodulation limited by residual intensity modulation, with an arbitrary phase difference between the angle modulation and the intensity modulation. The frequency dependent terms are only a small correction for closely spaced tones.

\begin{tabular}{|c|c|c|}
\hline & PM & FM \\
\hline \hline OIP2 & $2\left|Z_{\text {out }}\right| \frac{i_{d c}^{2}}{T}\left|\frac{A f_{1}}{\Gamma \cos (\phi / 2)}\right|^{2}$ & $2\left|Z_{\text {out }}\right| \frac{i_{d c}^{2}}{T}\left|\frac{A f_{1}}{\Gamma \cos (\phi / 2)}\right|^{2}$ \\
\hline Shot noise $S F D R_{2}$ & $\frac{A f_{1}}{\Gamma|\cos (\phi / 2)|} \sqrt{\frac{\Re P_{\text {opt }}}{q B}}$ & $\frac{A f_{1}}{\Gamma|\cos (\phi / 2)|} \sqrt{\frac{\Re P_{\text {opt }}}{q B}}$ \\
\hline Phase noise $S F D R_{2}$ & $\frac{f_{1}}{\Gamma|\cos (\phi / 2)|} \sqrt{\frac{\pi}{2 \Delta \nu B}}$ & $\frac{f_{1}}{\Gamma|\cos (\phi / 2)|} \sqrt{\frac{\pi}{2 \Delta \nu B}}$ \\
\hline OIP3 & $8\left|Z_{\text {out }}\right| \frac{i_{\text {dc }}}{T}\left(\frac{A f_{1}}{\Gamma}\right)^{2} \frac{f_{1}}{\mid 2 f_{1}+f_{2} \exp [j 2 \phi \mid]}$ & $8\left|Z_{\text {out }}\right| \frac{i_{\text {dc }}^{2}}{T}\left(\frac{A f_{1}}{\Gamma}\right)^{2} \frac{f_{2}}{\mid 2 f_{1}+f_{2} \exp [j 2 \phi \mid]}$ \\
\hline Shot noise $S F D R_{3}$ & $\left(\frac{4 \Re P_{\text {opt }}}{q B}\left(\frac{A f_{1}}{\Gamma}\right)^{2} \frac{f_{1}}{\left|2 f_{1}+f_{2} \exp [j 2 \phi]\right|}\right)^{\frac{2}{3}}$ & $\left(\frac{4 \Re P_{\text {opt }}}{q B}\left(\frac{A f_{1}}{\Gamma}\right)^{2} \frac{f_{2}}{\left|2 f_{1}+f_{2} \exp [j 2 \phi]\right|}\right)^{\frac{2}{3}}$ \\
\hline Phase noise $S F D R_{3}$ & $\left(\frac{2 \pi}{\Delta \nu B}\left(\frac{A f_{1}}{\Gamma}\right)^{2} \frac{f_{1}}{\left|2 f_{1}+f_{2} \exp [j 2 \phi]\right|}\right)^{\frac{2}{3}}$ & $\left(\frac{2 \pi}{\Delta \nu B}\left(\frac{A f_{1}}{\Gamma}\right)^{2} \frac{f_{2}}{\left|2 f_{1}+f_{2} \exp [j 2 \phi]\right|}\right)^{\frac{2}{3}}$ \\
\hline
\end{tabular}

Figure 2.9: $O I P 3$ and $\mathrm{SFDR}_{3}$ for an ideal discriminator for different values of residual intensity modulation, assuming closely spaced tones around $2 \mathrm{GHz}, 5 \mathrm{~mA}$ of current per detector $\left(i_{d c}=10 \mathrm{~mA}\right), 50 \mathrm{ohm}$ impedance, and 0.5 amplitude bias, $T=0.25$.
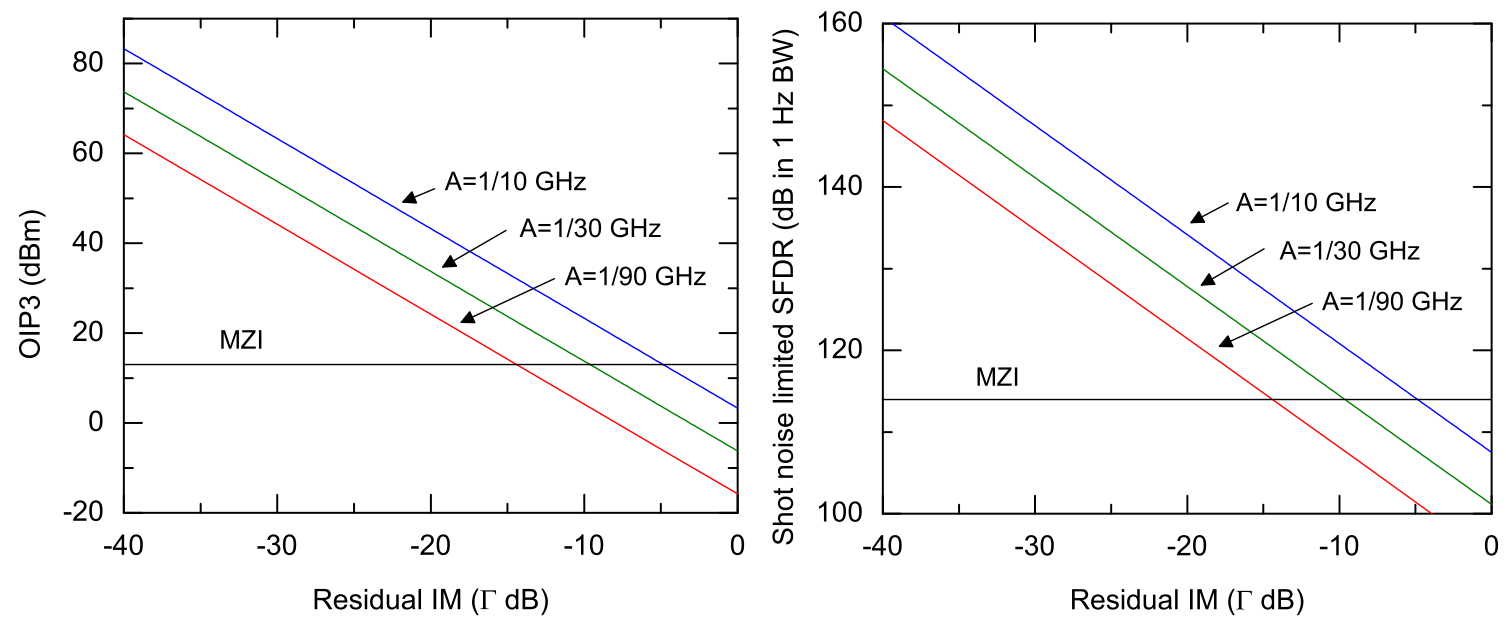
Figure 2.10: OIP3 for complementary linear-field discriminators for different slope values and fiber dispersion, assuming standard SMF, with $D=-20 \mathrm{ps}^{2} / \mathrm{km}$, closely spaced tones around $2 \mathrm{GHz}, 5 \mathrm{~mA}$ of current per detector $\left(i_{d c}=10 \mathrm{~mA}\right), 50 \mathrm{ohm}$ impedance, and 0.5 amplitude bias, $T=0.25$.

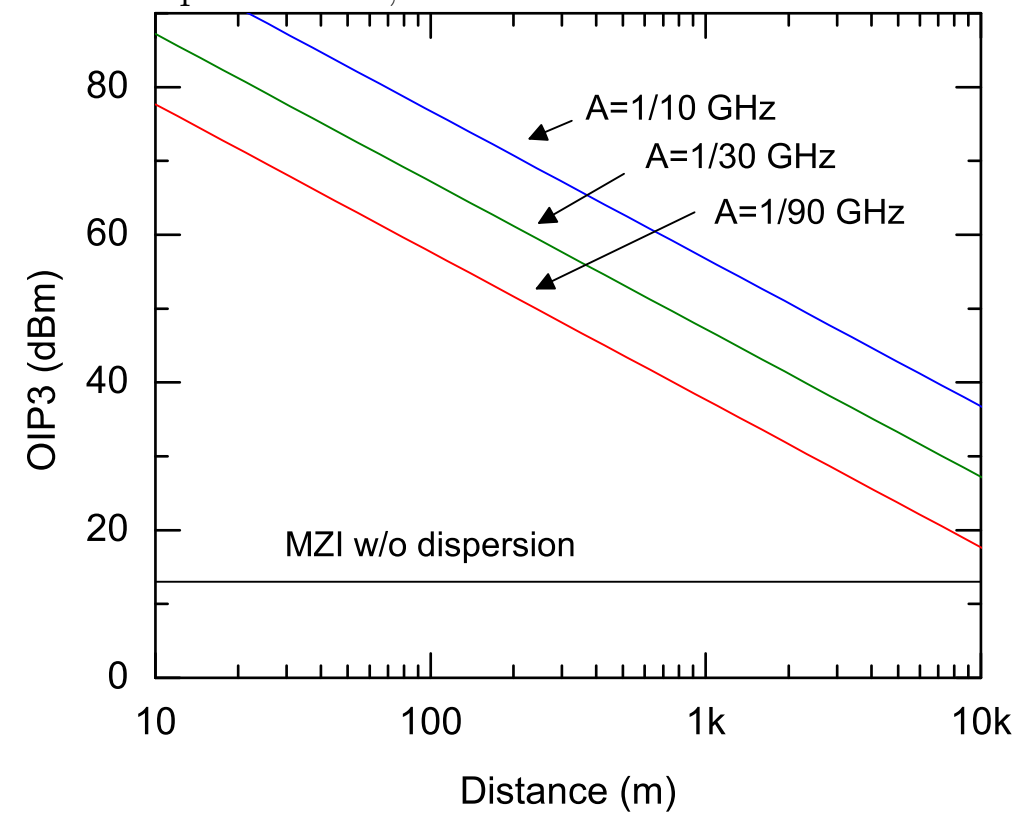

\subsection{Summary}

In this chapter, I have proven theoretically that complementary linear-field discriminators, if implementable with real optical filters, can potentially lead to microwave photonic links with very high dynamic range. Table 2.4 summarizes the noise figure metric in the shot noise and phase noise limited regimes, table 2.5 gives limits on the spurious free dynamic range by filter curvature, and table 2.6 gives the SFDR limited by residual intensity modulation. Assuming the link is limited by photodetector current rather than optical power, I find that the gain and noise figure both benefit from low biasing the discriminators. In the next chapter, the arbitrary filter model derived here will be used to evaluate physical implementions of the discriminators, to predict the limits of their performance. 


\section{Chapter 3}

\section{Simulated filter performance}

Complementary linear-field demodulation can achieve high dynamic range if good approximations to the desired filter transfer functions can be physically realized. In recent years, there has been much work in devising microwave photonic filters $[8,63]$. As reviewed by [64], a systematic way that microwave photonic filters can be designed is by using techniques borrowed from the field of digital filters. One specifies the coefficients of the z-transform representation of the filter, and then uses a synthesis algorithm to map to optical components such as couplers, resonators, and delay lines. The problem of discriminator design reduces to one of choosing the best coefficients and then fabricating a filter which can implement them. This chapter is a refinement of work I first reported in [65] on designing FIR filters for PM/FM-DD links. Links are implemented using different discriminator filters, and their performance is analyzed using a small signal model, a full signal model, and a numerical simulation.

\subsection{Filter coefficients}

Finite impulse response (FIR) filters, with all zeros and no poles in their z-transform representations, may work well as FM discriminators because symmetric FIRs can be designed to have exactly linear phase, and the theory shows that the filter's phaselinearity affects the link's linearity. In this and following sections, I present sets of FIR coefficients, chosen with different criteria, and compare their performance as discriminators in photonic links.

My initial comparison is made between different 10th order (or length 11) symmetric FIR filters. The transfer function for the positive slope filter goes from 0 to 1 within half the filter's free spectral range (FSR), which is the domain of normalized angular frequencies from 0 to $\pi$. The transfer function for the complemantary filter with negative slope goes from 1 to 0 over the same domain. The optical carrier is biased at the midband angular frequency $\pi / 2$, which is half-field bias.

I chose three sets of filter coefficients. The first two were chosen using an optimization routine with least-squares error minimization. Because it is difficult to match the transfer function over the full range, the first filter was optimized from 0.3 to 0.7. The second filter was optimized closer to the carrier from 0.45 to 0.55 . The third set 
Table 3.1: Filter coefficients for negative slope and positive slope, midband optimized, 10th order, FIR discriminators. Each filter is symmetric, so half the coefficients are duplicated. The symmetric filters are guaranteed to have linear phase. The first least squares fit is optimized for normalized frequencies 0.3 to 0.7 , and the second least squares fit is optimized for normalized frequencies 0.45 to 0.55 . The coefficients for the maximally linear filter are from the cited reference. All three filters are Type I linear phase FIR filters (odd-length, symmetric).

\begin{tabular}{|c|c|c|c|c|c|c|}
\hline Coefficients & \multicolumn{2}{|c|}{ Least-Squares 1 } & \multicolumn{2}{|c|}{ Least-Squares 2 } & \multicolumn{2}{c|}{ Maximally linear } \\
\hline \hline & + Slope & - Slope & + Slope & - Slope & + Slope & - Slope \\
\hline$a_{0}, a_{10}$ & -0.00109 & 0.00109 & -0.00076 & 0.00076 & $\frac{-3}{5(2 \pi) 2^{7}}$ & $\frac{3}{5(2 \pi) 2^{7}}$ \\
\hline$a_{1}, a_{9}$ & 0 & 0 & 0 & 0 & 0 & 0 \\
\hline$a_{2}, a_{8}$ & -0.01186 & 0.01186 & -0.01045 & 0.01045 & $\frac{-25}{3(2 \pi) 2^{7}}$ & $\frac{25}{3(2 \pi) 2^{7}}$ \\
\hline$a_{3}, a_{7}$ & 0 & 0 & 0 & 0 & 0 & 0 \\
\hline$a_{4}, a_{6}$ & -0.18929 & 0.18929 & -0.18669 & 0.18669 & $\frac{-150}{(2 \pi) 2^{7}}$ & $\frac{150}{(2 \pi) 2^{7}}$ \\
\hline$a_{5}$ & 0.50000 & 0.50000 & 0.50000 & 0.50000 & $\frac{1}{2}$ & $\frac{1}{2}$ \\
\hline
\end{tabular}

of filter coefficients was chosen using the maximally linear criterium. This criterium was developed by B. Kumar and S.C. Dutta Roy in [66-68] for application in digital differentiator filters. The maximally linear criterium fixes a number of derivatives of the transfer function at a chosen frequency, guaranteeing high accuracy around a small frequency band. If this band is comparable to the bandwidth of modulation, overall I expect high linearity. The intuition for these choices were based on the error bounds in the derived masks, which has tighter constraints close to the carrier.

The three sets of filter coefficients are presented in Table 3.1 on page 42. The transfer functions for the filters are plotted in Figure 3.1 on page 43. All three filter designs appear very linear on the full scale, except for the curvature at the frequencies furthest away from the carrier. The figure also shows the deviation of the transfer functions from the ideal linear ramp plotted on a logarithmic scale. For reference, I show the cubic curvature masks for $\varepsilon_{3, \max }=0.01,0.001$, and 0.0001 . The first least-squares fit is optimized over a wider range of frequencies, but the second-fit has much smaller deviation closer to the carrier. The maximally-linear fit has the smallest bandwidth that is optimized, but it is the closest to the ideal filter over that bandwidth. This observation suggests a tradeoff of linearity and bandwidth so that the filter coefficients can be adjusted to best serve the modulation frequencies of interest.

The transfer functions were analyzed using the small signal model presented in the previous chapter. The code is included in the appendix, Section A.1. Fig. 3.2 shows OIP3 versus modulation frequency for links using each of the three sets of filters. The link is more linear for lower modulation frequencies, and gets worse for large modulation frequencies. The OIP3 from the maximally linear filter is smoothly 
Figure 3.1: Transfer functions for the FIR discriminators optimized at midband.
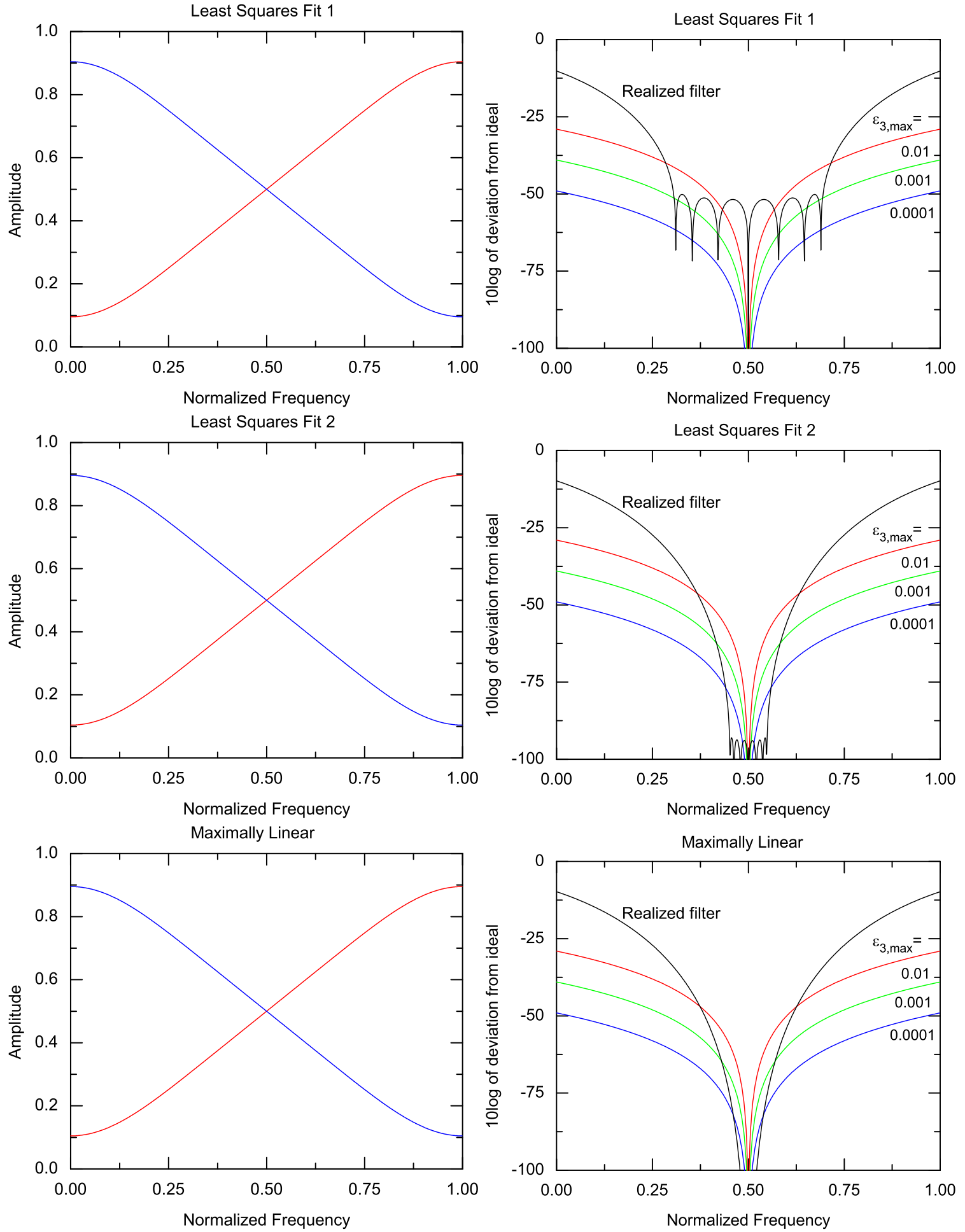
Figure 3.2: Simulated OIP3 for the three different 10th order FIR filter sets optimized at midband versus normalized modulation frequency. The photocurrent is scaled for $10 \mathrm{~mA}$ total photocurrent ( $5 \mathrm{~mA}$ per detector). The filter is more linear for lower modulation frequencies, and gets worse for large modulation frequencies. For the least squares fit filters, the local minima for certain modulation frequencies are apparent in the plot.

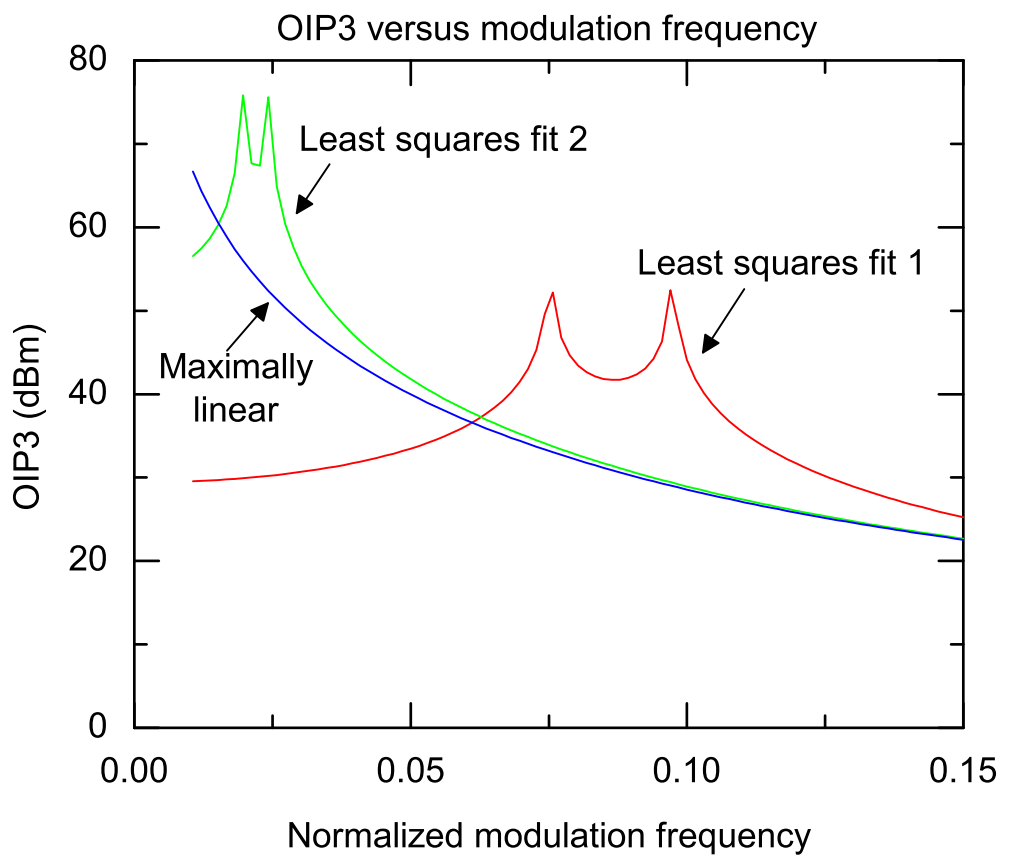

varying and monotonically decreasing as the modulation frequency increases. These are desirable properties when doing further trade-off analysis for the discriminators, so later sections will employ the maximally-linear filters.

In these designs, there is no second-order nonlinearity for the link. However, if the detection is not perfectly balanced, then there is some second-order distortion. In the simulation, I find that OIP2 does not depend on the modulation frequency, but does scale with the common-mode rejection ratio (CMRR) of the detection. Fig. 3.3 shows the OIP2 versus CMRR.

\subsection{Scaling with filter order}

Next, I study the scaling with filter order of the linearity of a link employing maximallylinear filters as the discriminator filters. Table 3.2 on page 45 gives the coefficients for 2nd, 6th, 10th, 14th, and 18th order maximally linear filters optimized at half-band [67]. The distortion of a link using each of the filters was simulated. Fig. 3.4 shows the scaling of OIP3 with the filter order versus modulation frequency. As expected, higher order filters give larger OIP3 than lower-order filters. The linearity has a large improvement over a balanced MZI for a given photocurrent. In a physical link, the highest values of OIP3 ( $>40 \mathrm{dBm})$ may be limited by photodetector nonlinearities. 
Figure 3.3: Simulated OIP2 for the 10th order maximally linear FIR filter set optimized at midband versus common mode rejection ratio. The CMRR is given in decibels of current suppressed. The photocurrent is scaled for $10 \mathrm{~mA}$ total photocurrent ( $5 \mathrm{~mA}$ per detector). The normalized modulation frequency is 0.03 , but no dependence of OIP2 on modulation frequency was observed. For infinite CMRR, the OIP2 value was limited by the numerical precision of the calculation.

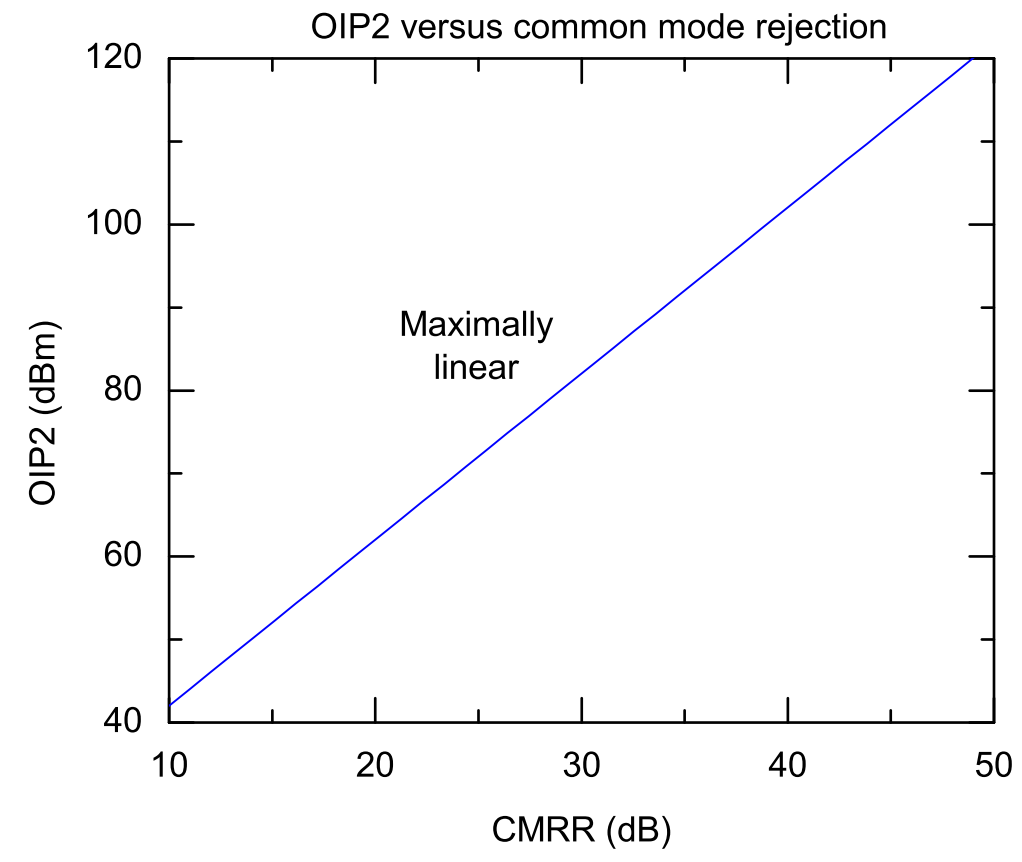

Table 3.2: Filter coefficients for the 2nd, 6th, 10th, 14th, and 18th order maximally linear filters in z-transform representation. Each filter is symmetric, so half the coefficients are duplicated. The coefficients given are for the positive slope filters. For negative slope filters, the even-numbered coefficients have opposite sign.

\begin{tabular}{|c|c|c|c|c|c|}
\hline Coefficients/Order & 2 & 6 & 10 & 14 & 18 \\
\hline \hline$a_{n / 2 \pm 9}$ & & & & & $\frac{-35 / 9}{(2 \pi) 2^{15}}$ \\
\hline$a_{n / 2 \pm 8}$ & & & & & 0 \\
\hline$a_{n / 2 \pm 7}$ & & & & $\frac{-10 / 7}{(2 \pi) 2^{11}}$ & $\frac{-405 / 7}{(2 \pi) 2^{15}}$ \\
\hline$a_{n / 2 \pm 6}$ & & & & 0 & 0 \\
\hline$a_{n / 2 \pm 5}$ & & & $\frac{-3 / 5}{(2 \pi) 2^{7}}$ & $\frac{-98 / 5}{(2 \pi) 2^{11}}$ & $\frac{-2268 / 5}{(2 \pi) 2^{15}}$ \\
\hline$a_{n / 2 \pm 4}$ & & & 0 & 0 & 0 \\
\hline$a_{n / 2 \pm 3}$ & & $\frac{-1 / 3}{(2 \pi) 2^{3}}$ & $\frac{-25 / 3}{(2 \pi) 2^{7}}$ & $\frac{-490 / 3}{(2 \pi) 2^{11}}$ & $\frac{-8820 / 3}{(2 \pi) 2^{15}}$ \\
\hline$a_{n / 2 \pm 2}$ & & 0 & 0 & 0 & 0 \\
\hline$a_{n / 2 \pm 1}$ & $\frac{-1}{2 \pi}$ & $\frac{-9}{(2 \pi) 2^{3}}$ & $\frac{-150}{(2 \pi) 2^{7}}$ & $\frac{-2450}{(2 \pi) 2^{11}}$ & $\frac{-39690}{(2 \pi) 2^{15}}$ \\
\hline$a_{n / 2}$ & $\frac{1}{2}$ & $\frac{1}{2}$ & $\frac{1}{2}$ & $\frac{1}{2}$ & $\frac{1}{2}$ \\
\hline
\end{tabular}


Figure 3.4: Simulated OIP3 for maximally linear FIR filters, of different order, optimized at midband versus normalized modulation frequency. The photocurrent is scaled for $10 \mathrm{~mA}$ total photocurrent (5 mA per detector).

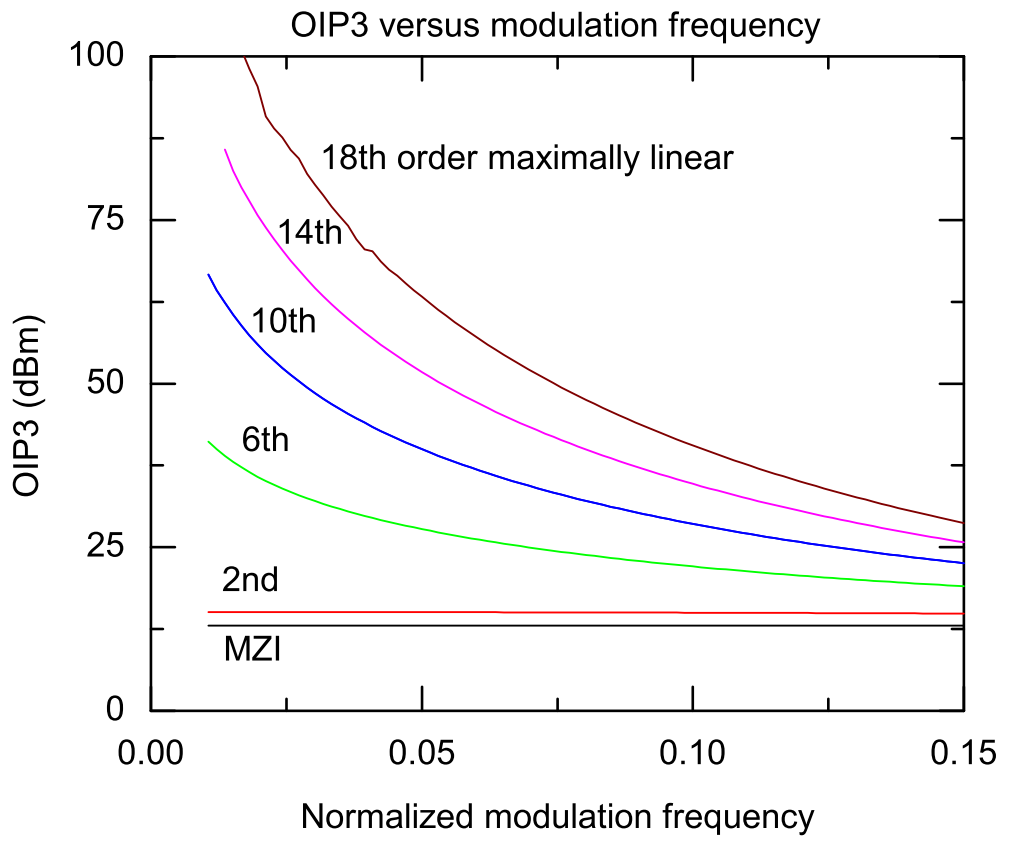

To study the scaling of the spurious-free dynamic range with filter order, I give example parameters for a phase-modulated link based on currently available commerical components. The full free-spectral range of the filter is chosen to be $200 \mathrm{GHz}$, and the modulation frequency, $5 \mathrm{GHz}$. (The normalized frequency is therefore 0.05 ). The transmitter consists of an external-cavity laser with a $100 \mathrm{kHz}$ linewidth, and a lithium niobate phase-modulator with halfwave voltage $V_{\pi}=3 \mathrm{~V}$. The input and output loads are assumed to be $50 \Omega$. The balanced detector handles up to $50 \mathrm{~mA}$ of dc photocurrent, or $25 \mathrm{~mA}$ per detector.

Simulating these values, we end up with a link with gain of $-5.6 \mathrm{~dB}$ and noise figure of $10.5 \mathrm{~dB}$. The gain and noise figure will be similar for a link with a MZI discriminator. The gain and noise figure can be improved by increasing the modulation efficiency (decreasing $V_{\pi}$ ), or increasing the detector power handling. The resulting SFDR versus filter order is shown in Fig. 3.5. The phase noise limited and shot noise limited approximations are shown in the plot along with the full noise figure model. The SFDR is close to being limited by the laser phase noise: there is a difference of about $2 \mathrm{~dB}$. The noise figure is primarily limited by the rf link-loss, and secondly by the linewidth of the laser. Both would need to be improved to get to shot noise limited performance.We also find that to some extent, one can trade-off between SFDR and noise figure by adjusting the filter's FSR. This is shown in Fig. 3.6. With a fixed modulation frequency, making the FSR larger reduces the gain, thus making the NF worse. However, the filter is more linear closer to the carrier, so the SFDR can be improved. Like the MZI, the SFDR for the 2nd order order filter is nearly independent of its FSR. 
Table 3.3: Simulation parameters

\begin{tabular}{|c|c|}
\hline Parameter & Value \\
\hline \hline$f_{1}(\mathrm{GHz})$ & 5.0 \\
\hline$f_{2}(\mathrm{GHz})$ & 5.005 \\
\hline Input/Output Impedances $(\Omega)$ & 50 \\
\hline Modulation efficiency $\left(V_{\pi}\right)$ & 3 \\
\hline Laser linewidth $(\mathrm{kHz})$ & 100 \\
\hline Filter free spectral range $(\mathrm{GHz})$ & 200 \\
\hline Optical power before filters $(\mathrm{mW})$ & 250 \\
\hline Optical power incident upon each detector $(\mathrm{mW})$ & 31.25 \\
\hline Photodetector responsivity $(\mathrm{A} / \mathrm{W})$ & 0.8 \\
\hline DC photocurrent per detector $(\mathrm{mA})$ & 25 \\
\hline Third-order IMD $(\mathrm{GHz})$ & 4.995 \\
\hline Second harmonic $(\mathrm{GHz})$ & 10 \\
\hline
\end{tabular}

The SFDR with the 10th order filter, and $200 \mathrm{GHz}$ FSR has a SFDR of $129 \mathrm{~dB}$ in 1 $\mathrm{Hz}$ bandwidth, which is better than the state-of-the-art links appearing in literature. The SFDR increases by $8 \mathrm{~dB}$ for every increase of 4 for the filter order. This suggests a great benefit from photonic integration: the link's SFDR scales with the square of the filter order!

\subsection{Numerical link simulation}

Finally, I compare the small-signal model to the full-signal model and numerical link simulations to understand the limitations of the small-signal model. The fullsignal model is the infinite summation given in (2.9). For the numerical model, the signal at the output of the link is simulated by creating a time domain waveform, $e(t) \propto \exp \left[2 \pi f_{c} t+2 \pi \eta \int i(t)\right]$, performing a fast Fourier transfer (FFT), weighting the frequency domain waveform by a given filter transfer function, performing an inverse FFT and squaring the time domain waveform to obtain the photocurrent. The simulation process is illustrated in figure 3.7. The code for all three simulations are included in the Appendix. The simulation code includes the effect of imperfect common-mode rejection from the balanced photodetection.

The plot in Fig. 3.8 shows the link response of a $5 \mathrm{GHz}$ PM-DD link using the link parameters discussed in the previous section. The fundamental and third-order intermodulation distortion powers are plotted versus input power. The noise floor is calculated using the small-signal model. As can be seen in the plot, the large-signal analytical model and the numerical simulation using FFTs closely track each-other.We find that the numerical simulation is much more computationally efficient, taking an order of magnitude less time to execute.

For small modulation power, the three models match up. For large modulation power, the distortion of the link increases much faster than the $30 \mathrm{~dB}$ per decade suggested by the small-signal model. This can be explained by observing that in 
Figure 3.5: Spurious free dynamic range versus filter order for $5 \mathrm{GHz}$ PM-DD links using maximally linear filters and $200 \mathrm{GHz}$ FSR. The link parameters are given in Table 3.3 on page 47 .

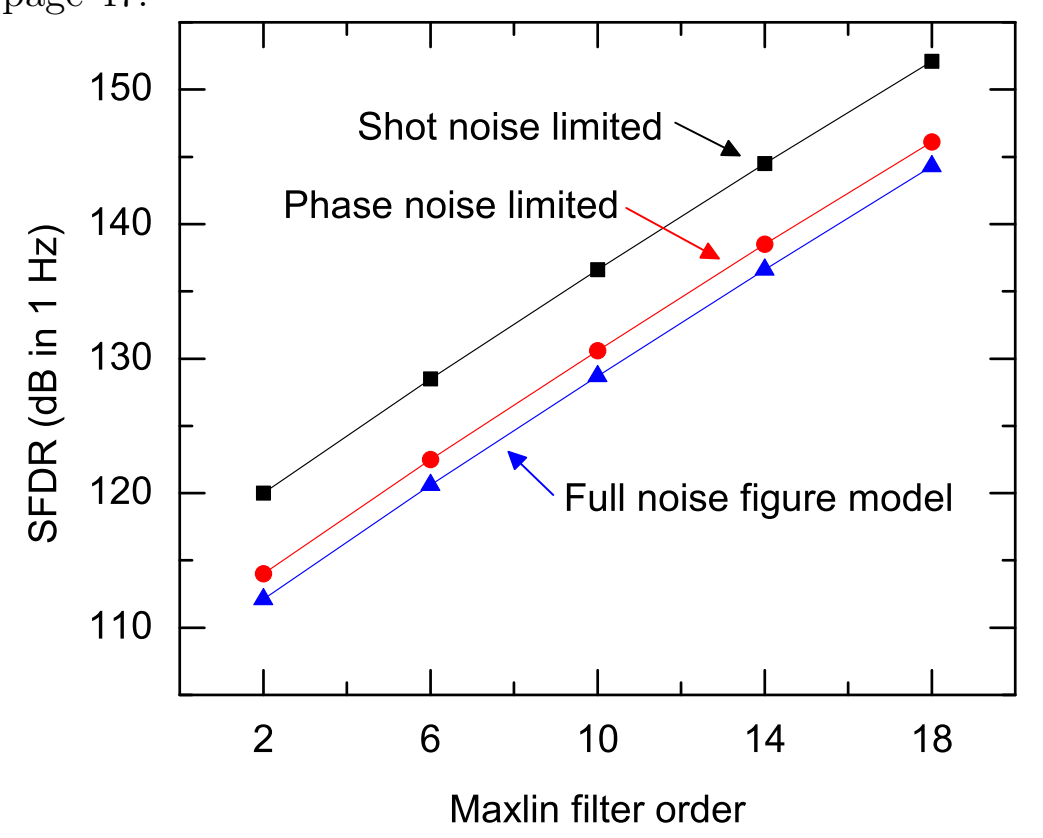

Figure 3.6: Spurious free dynamic range for $5 \mathrm{GHz}$ PM-DD links using maximally linear filters for various FSR.

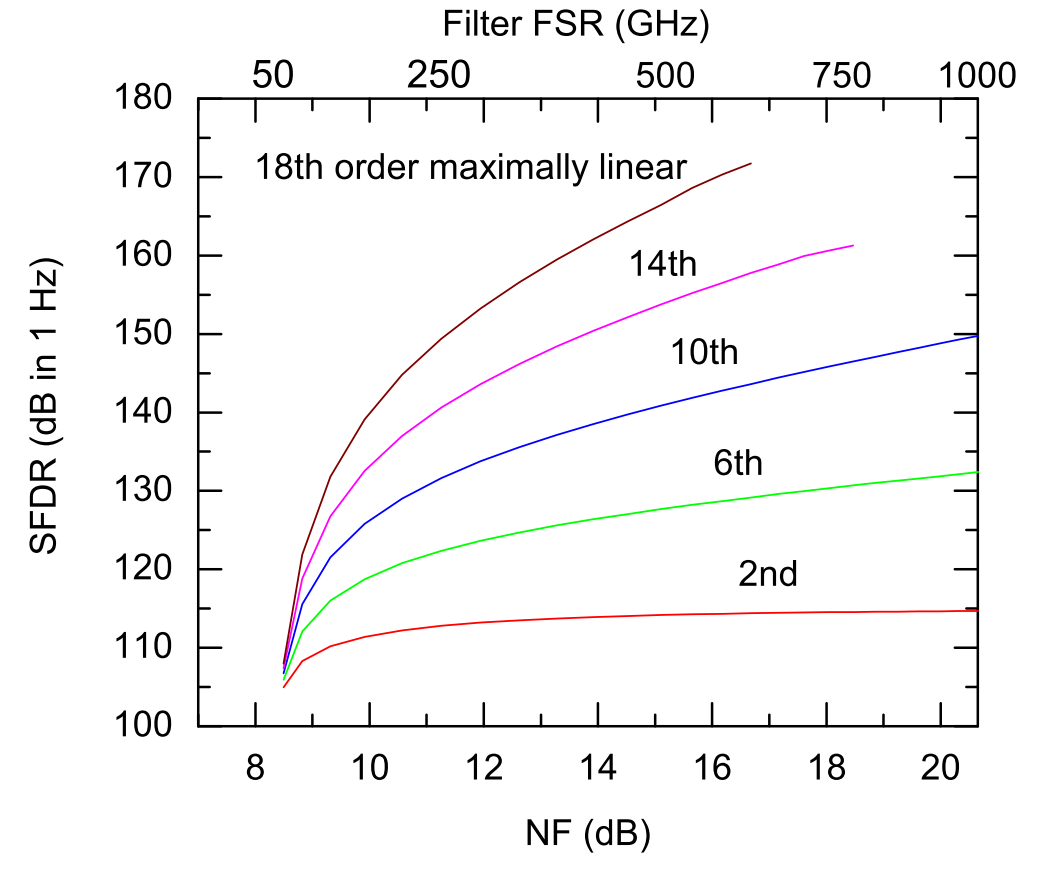


Figure 3.7: Numerical model of a PM-DD or FM-DD photonic link with two discriminator filters and balanced detection

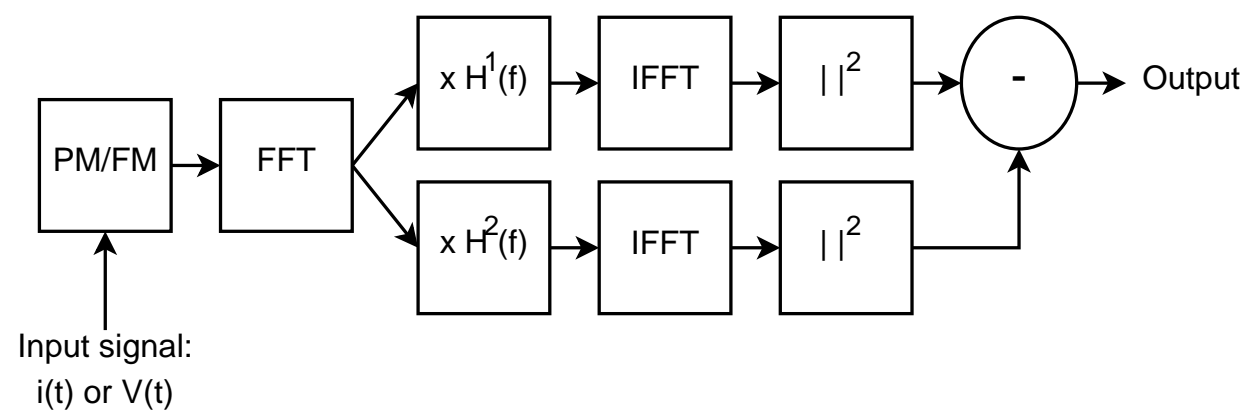

frequency or phase modulation, the frequency deviation of the carrier increases with modulation depth, so more optical power is spread into higher order sidebands. For high modulation depths, most of the optical power lies outside of the range of frequencies for which the filter is optimized, creating more distortion than for low modulation depths. For the link under discussion, the models for IMD3 begin to deviate around $1 \mathrm{~mW}$ of input power, or more precisely, the two values are $3 \mathrm{~dB}$ off when the input power is $1.6 \mathrm{dBm}$. This gives a phase modulation depth of 0.4 or a frequency modulation depth of $2 \mathrm{GHz}$. This makes sense in the context of Carson's bandwidth rule for frequency modulation: the bandwidth occupied by the modulated signal starts to have a noticeable increase once the modulation frequency and the frequency modulation depth are of the same order of magnitude.

The faster than $30 \mathrm{~dB}$-per-decade increase in distortion power has consequences for the actual spurious free dynamic range seen by the system. Fig. 3.10 compares the small-signal approximation to the SFDR with SFDR values calculated by finding the intercept of the IMD3 with the noise in a given bandwidth. Large bandwidths will be unequally affected by the sideband spill-over effect. A link designer needs to be cognizant of the full-signal model in order to accurately predict the spurious signal levels seen.

\subsection{Summary}

We have demonstrated by simulation that frequency and phase modulated microwavephotonic links with very high linearity are obtainable by using FIR optical filters to perform demodulation. Links using filters designed with the maximally linear criteria greatly exceed the linearity performance of an MZI. Although this chapter did not simulate the performance of IIR filters as discriminators, in general it is expected that more closely matched filters could be implemented with fewer stages with an IIR architecture. We have observed that linearity degrades for high modulation depths as power is spread into high-order optical sidebands far from the optical carrier. We find that a tenth-order FIR filter designed using the maximally linear criteria can obtain a $129 \mathrm{~dB} \cdot \mathrm{Hz}^{2 / 3} \mathrm{SFDR}$ with $50 \mathrm{~mA}$ of photocurrent. The SFDR scales with the square 
Figure 3.8: Link response versus input power for a 5 GHz PM-DD link using tenthorder maximally linear filters. The link parameters are given in the text.

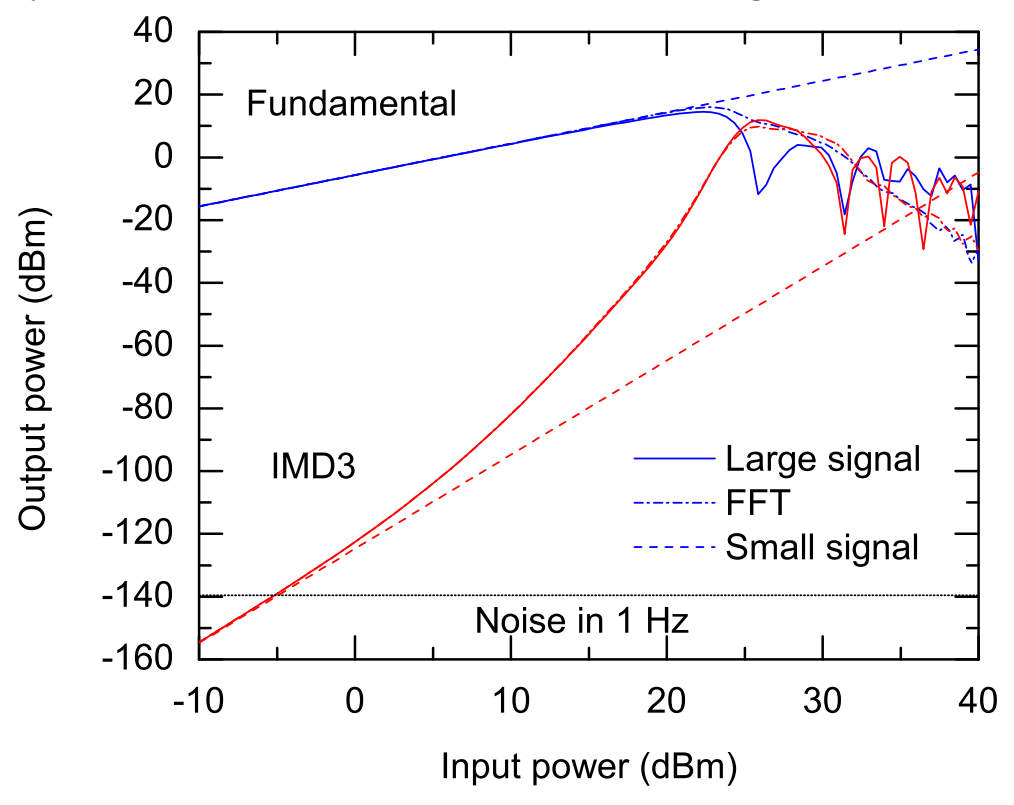

Figure 3.9: Link response versus input power for a $5 \mathrm{GHz}$ PM-DD link using maximally linear filters of different order.

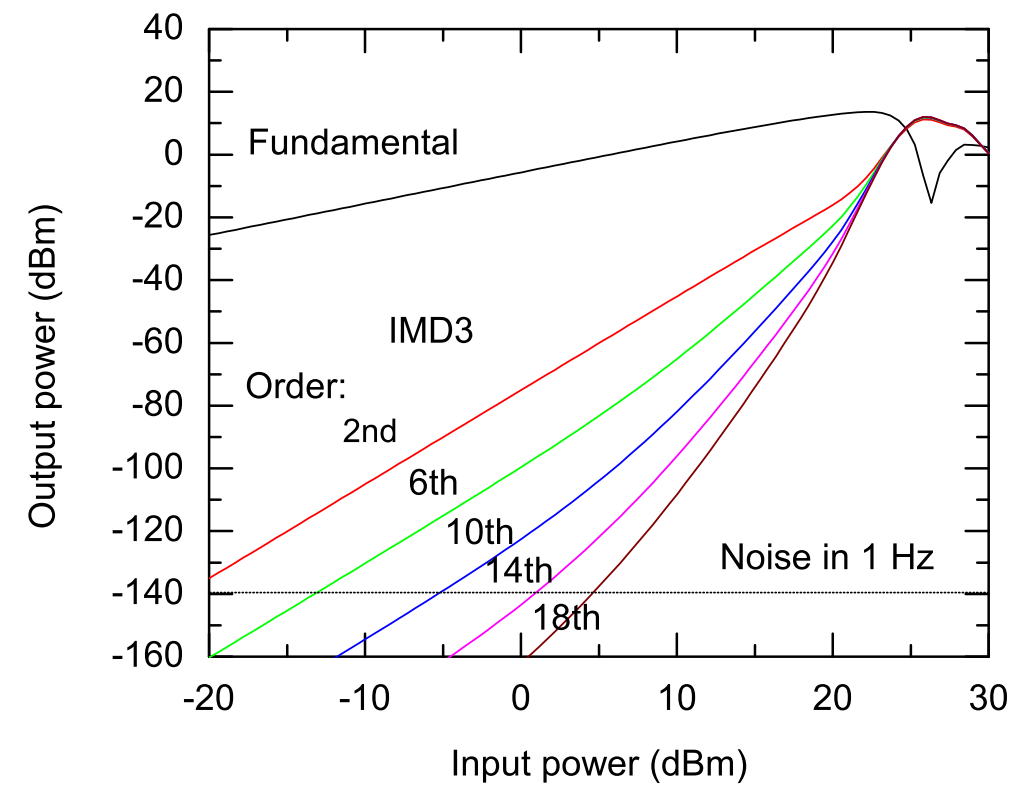


Figure 3.10: Spurious free dynamic range versus bandwidth for $5 \mathrm{GHz}$ PM-DD links using maximally linear filters of different orders.

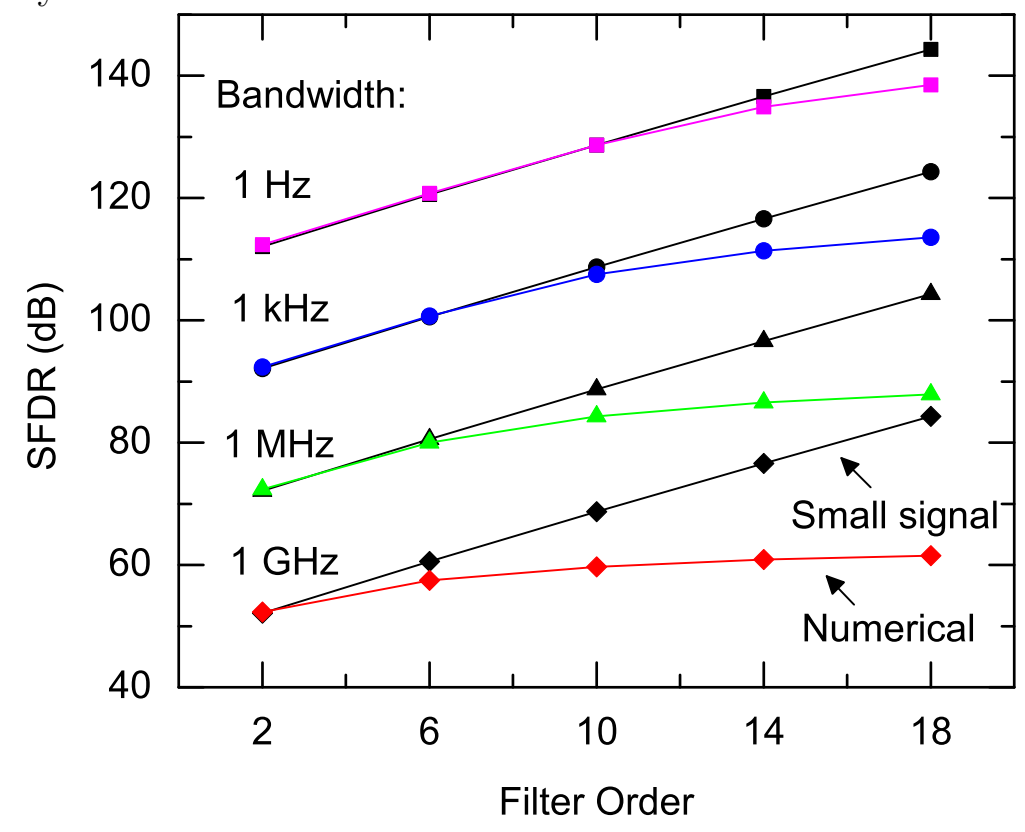

of the FIR filter order, suggesting a benefit to increasing photonic integration. 


\section{Chapter 4}

\section{Phase modulation experiments}

Using complementary linear-field discriminator filters, we believe we have demonstrated PM-DD and FM-DD links with the highest linearities which have been published thus far, as measured by third-order and second-order output-intercept points (OIP3 and OIP2) normalized to a fixed, photodetector-limited photocurrent. Our discriminator filters are fabricated in a low-loss silica-on-silicon, planar-lightwave-circuits (PLC) process at Alcatel-Lucent Bell Laboratories. We report link measurements using both a cascaded MZI FIR lattice filter and a ring assisted MZI (RAMZI) IIR filter, and with both phase modulation and frequency modulation.

The discriminators are based on two architectures: a cascaded MZI FIR lattice filter [69] and a ring assisted MZI (RAMZI) IIR filter [70]. For both types of discriminators, we demonstrate $>6 \mathrm{~dB}$ improvement in the link's third-order output intercept point (OIP3) over a MZM link. We show that the links have low second-order distortion when using balanced detection. Using high optical power, we demonstrate an OIP3 of $39.2 \mathrm{dBm}$. We also demonstrate $4.3 \mathrm{~dB}$ improvement in signal compression.

\subsection{Planar lightwave circuit filters}

A discriminator filter approximating the ideal complementary linear-field response can be constructed using silica-on-silicon planar lightwave circuits (PLC) [71]. The transform function of an FIR filter can be realized in PLC with just MZIs and directional couplers. One implementation of a multi-stage optical FIR filter in PLC is the lattice filter [64]. The lattice filter architecture has a low-loss passband and requires only $\mathrm{N}+1$ couplers for an Nth order filter, which are advantages over other optical filter architectures. The lattice filter architecture is shown in Figure 4.1 on page 53, indicating for each stage the coupling coefficients, designated by $\kappa$, and the phase shifts, designated by $\varphi$. Each stage has a unit delay, $z^{-1}$. The dashed lines indicate additional filter stages omitted from the figure. A recursion relation exists that transforms between given filter coefficients and the corresponding coupling ratios and phase shifts [64]. The recursion relation for the tenth-order lattice filter design gives $2^{10}$, or 1024 solutions. For a tenth-order maximally linear discriminator filter, whose coefficients were given in the previous chapter in Table 3.2 on page 45 , one 
Figure 4.1: FIR lattice filter architecture

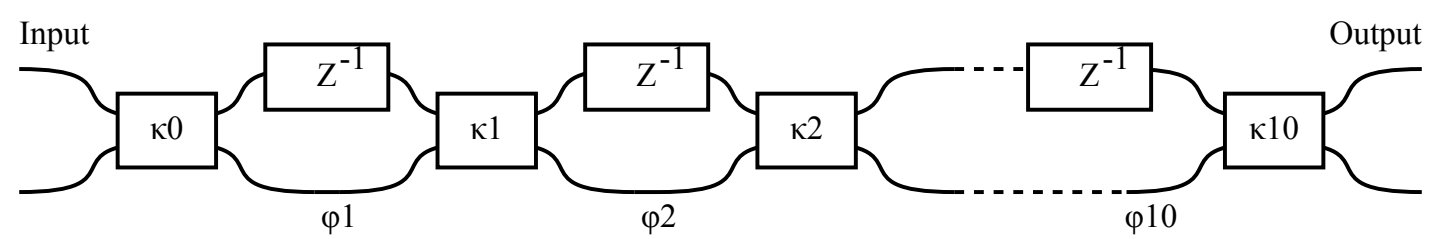

Table 4.1: Filter phase and coupler parameters for a tenth-order maximally linear discriminator filter in lattice filter form

\begin{tabular}{|c|c|c|c|c|}
\hline Phase shift & Value & Coupling ratio & Value & Tunable coupler phase \\
\hline \hline$\varphi_{1}$ & 0 & $\kappa_{0}$ & 0.674271 & 0.607391 \\
\hline$\varphi_{2}$ & $-\pi$ & $\kappa_{1}$ & 0.635460 & 0.648224 \\
\hline$\varphi_{3}$ & 0 & $\kappa_{2}$ & 0.837472 & 0.414954 \\
\hline$\varphi_{4}$ & $-\pi$ & $\kappa_{3}$ & 0.514751 & 0.770645 \\
\hline$\varphi_{5}$ & 0 & $\kappa_{4}$ & 0.918512 & 0.289487 \\
\hline$\varphi_{6}$ & 0 & $\kappa_{5}$ & 0.538578 & 0.746782 \\
\hline$\varphi_{7}$ & $-\pi$ & $\kappa_{6}$ & 0.918384 & 0.289721 \\
\hline$\varphi_{8}$ & 0 & $\kappa_{7}$ & 0.515217 & 0.770179 \\
\hline$\varphi_{9}$ & $-\pi$ & $\kappa_{8}$ & 0.837319 & 0.415161 \\
\hline$\varphi_{10}$ & $-\pi$ & $\kappa_{9}$ & 0.635828 & 0.647842 \\
\hline & & $\kappa_{10}$ & 0.325694 & 0.963443 \\
\hline
\end{tabular}

particular solution for the parameters in lattice filter form is listed in Table 4.1.

Up to tenth-order FIR lattice filters have been implemented in PLC for various applications. A research group at NTT laboratories has extensively explored tunable optical FIR lattice filters. Tunable coupling ratios are implemented by using symmetric Mach-Zehnder interferometers with thermal-optic phase shifters. A diagram of a tunable FIR filter is shown in Figure 4.2 on page 53. The intended application is dispersion compensation, but because the filters are tunable, they can be used for any filter transfer function desired, including discriminators.

The group has fabricated eight-order filters in silica with chromium heaters, with $50 \mathrm{GHz}$ FSR [69] and $200 \mathrm{GHz}$ FSR [72], and arrays of fifth-order filters with 50

Figure 4.2: Tunable PLC FIR lattice filter architecture

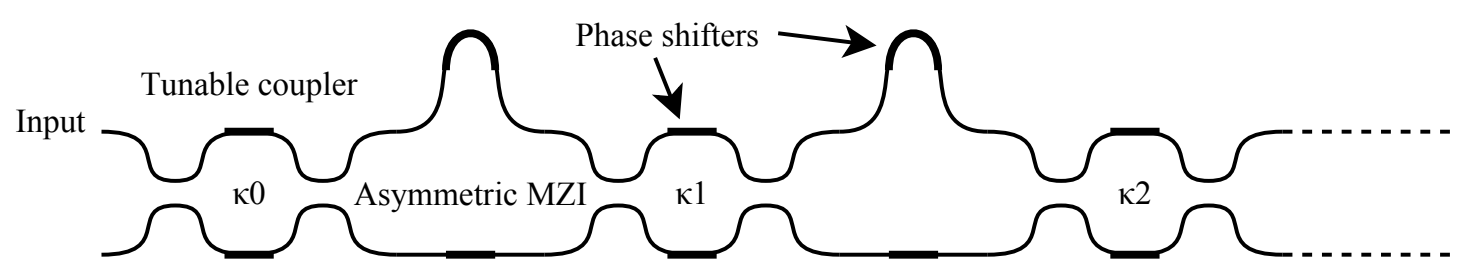


GHz FSR [73-75]. They claim control of the individual phase shifters to accuracy better than $0.01 \pi$ radians. For the fifth-order filters, to reduce the required bias power on the heaters, they use a phase-trimming technique that involves introducing heating induced stress. They have also proposed a $100 \mathrm{GHz}$ FSR filter in a reflection configuration to double its effective length [76].

A collaboration between Siemens, University of Kiel and IBM Research Zurich has implemented the same architecture on a more compact silicon oxynitride platform [77]. The applications include both EDFA gain equalization and dispersion compensation. They have demonstrated sixth, seventh and tenth-order filters with $100 \mathrm{GHz}$ FSR. The collaboration has explored a number of adaptive feedback approaches for setting the filter's phase shifters [78].

- Optical spectrum analysis [79]: They have used an optical spectrum analyzer to compare the amplified spontaneous emission spectrum to a desired intensity profile. A computer running the Levenberg-Marquart optimization algorithm (a modified Gauss-Newton algorithm) varies the power to the phase shifters until the desired profile is obtained.

- Electrical spectrum monitoring [80-82]: ESM is another feedback approach, where power at certain frequencies are used as a feedback mechanism. Pilot tones or knowledge about the signaling over the link determines the optimal choice of electrical filters.

- Eye opening [83-85]: An adaptive feedback approach for digital signals looks at an eye diagram and uses a Levenberg-Marquart optimization to maximize the eye opening.

- LMSE / minimize ISI [80, 82, 86-88]: Another method for digital signals uses minimization between the decision and signal as a feedback signal. These methods are not suitable for analog links.

For simplicity and cost, setting the filters coefficients without using a feedback system is desired. The IBM collaboration has developed a calibration procedure to produce a table look-up for tunable coupler and phase shifter responses versus applied tuning power [79]. The technique uses the OSA approach to iteratively tune all couplers to zero cross coupling. There is a procedure to individually characterize each tunable coupler and asymmetric MZI by measuring the output power versus tuning. The filter then can be set to a pre-calculated inverse system. An alternative calibration approach is given by the NTT group in [89] that does not require a feedback loop. The approach uses incoherent light to characterize each tunable coupler, and coherent light to characterize the asymmetric Mach Zehnders.

\subsection{Implementation and characterization}

As part of this program of research, two types of filters were fabricated and packaged at Alcatel-Lucent Bell Laboratories by Dr. Mahmoud Rasras: a cascaded MZI FIR 
Figure 4.3: (a) Filter stage for an FIR lattice filter (b) Filter stage for an IIR, RAMZI filter.

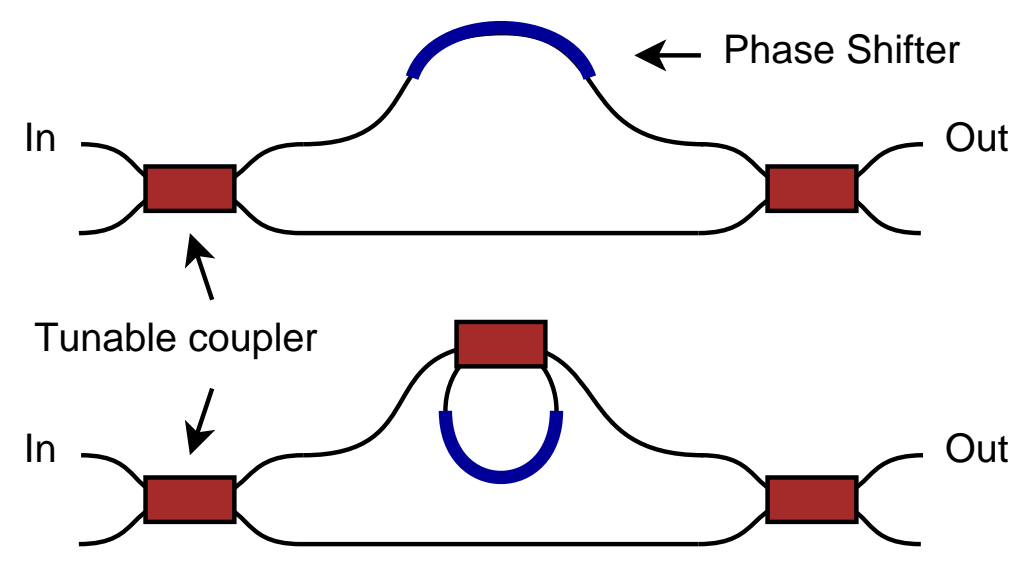

lattice filter and a ring assisted MZI (RAMZI) IIR filter. A single stage of each filter is illustrated in Fig. 4.3. These filters can be thermally tuned using chromium heaters to implement arbitrary filter transfer functions. The RAMZI IIR filter is a third-order filter with an all pass ring resonator structure coupled to the delay arm of an MZI. The FIR filter is a sixth-order filter with $120 \mathrm{GHz}$ free-spectral range. Our filter has 6 stages of symmetrical MZIs (switches) and asymmetrical MZIs (delay line interferometers) which are tunable using chromium heaters deposited on the waveguides. Figs. 4.4 and 4.5 show photographs of a fabricated and packaged FIR filter.

The experimental system for a phase-modulated link measurement is illustrated in Figure 4.6 on page 57. A polarization tracker is used at the output of the ECTL, and, where possible, the optical paths are polarization maintaining fiber. Two tunable RF sources are combined to modulate a commercial lithium niobate phase modulator to perform two-tone distortion measurements. We use a personal-computer-based analog output card to generate bias currents for the heaters to tune the transfer function of the discriminator. See Figs. 4.7 and 4.8. The paths between the filters and balanced detectors are trimmed to match delay and attenuation. For the FM measurements, the tunable laser, polarization controller, and phase modulator are replaced with the directly modulated FM laser.

\subsection{Link Results}

\subsubsection{Phase-modulated link with FIR filter}

We performed link measurements using the FIR filter and phase modulation. In our experiment the discriminator filter is dynamically tuned to minimize the link distortion. The filter has 13 degrees-of-freedom to adjust. If the filter is ideal, one can 
Figure 4.4: Photograph of single FIR filter with wiring board inside protective box.

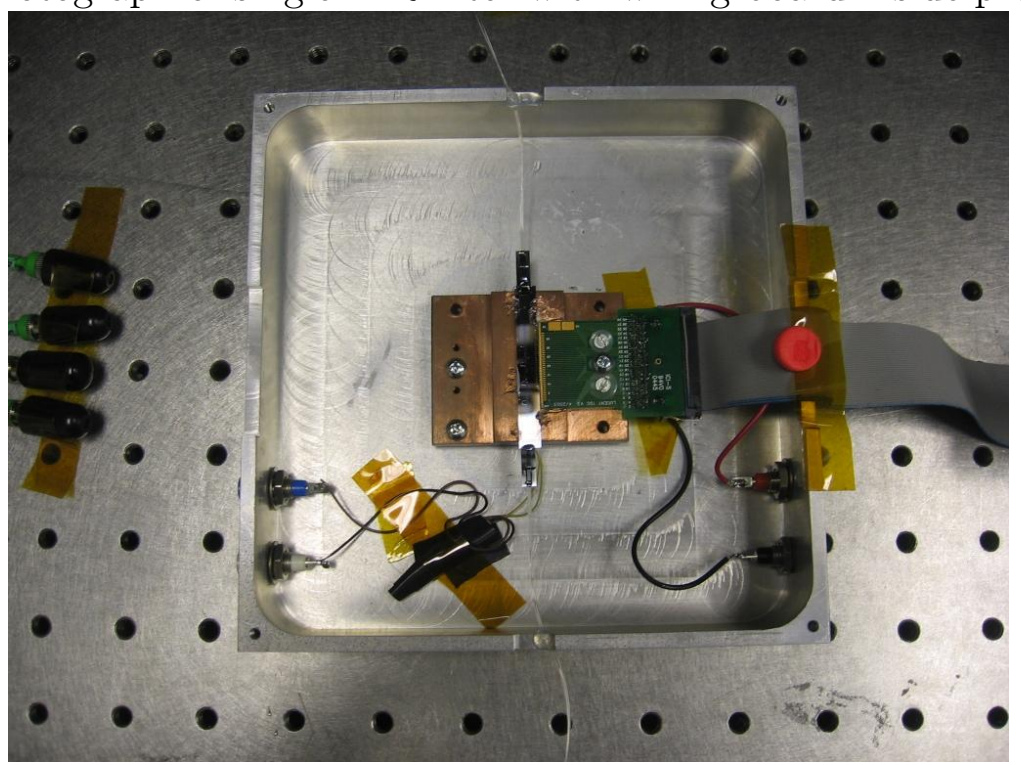

Figure 4.5: Photograph of single FIR filter mounted on heat sink.

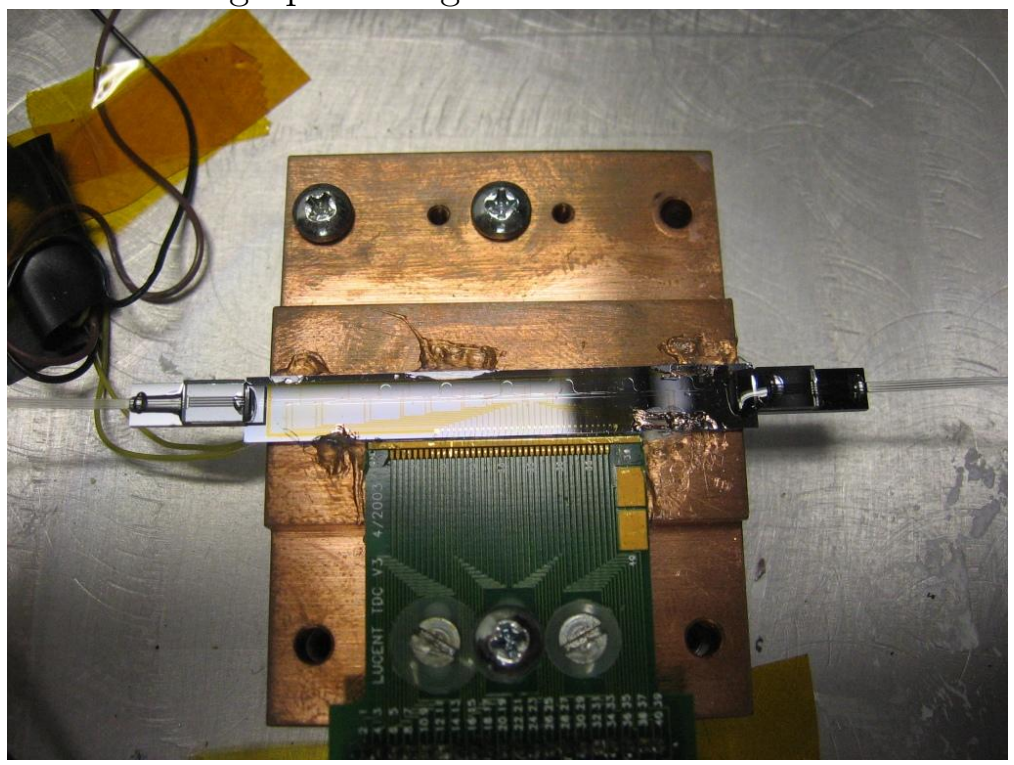


Figure 4.6: Diagram of the system used for characterization

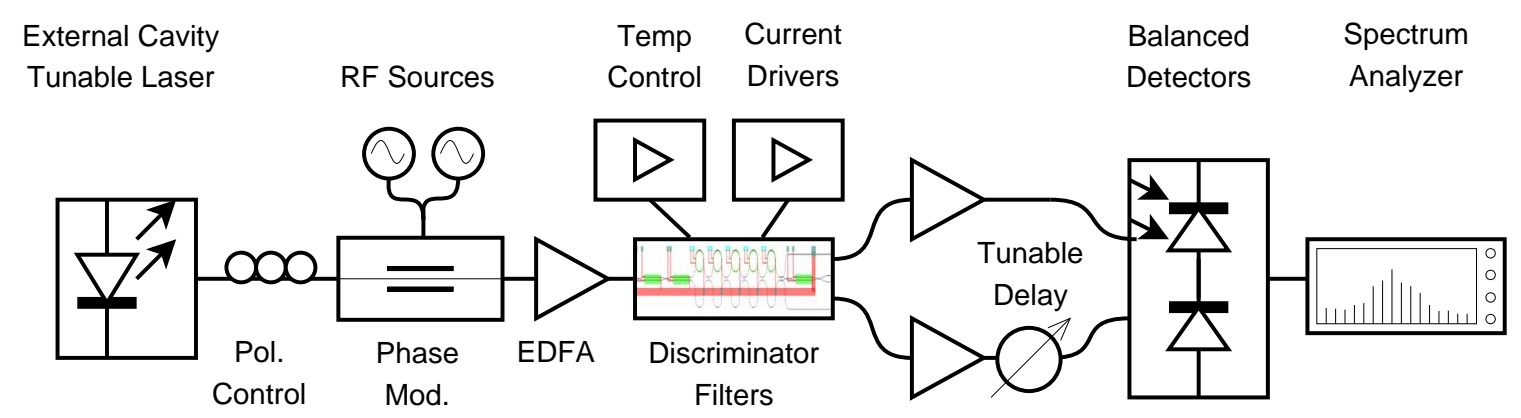

Figure 4.7: Photograph of current amplifier board to drive the chrome heaters on the tunable filters.

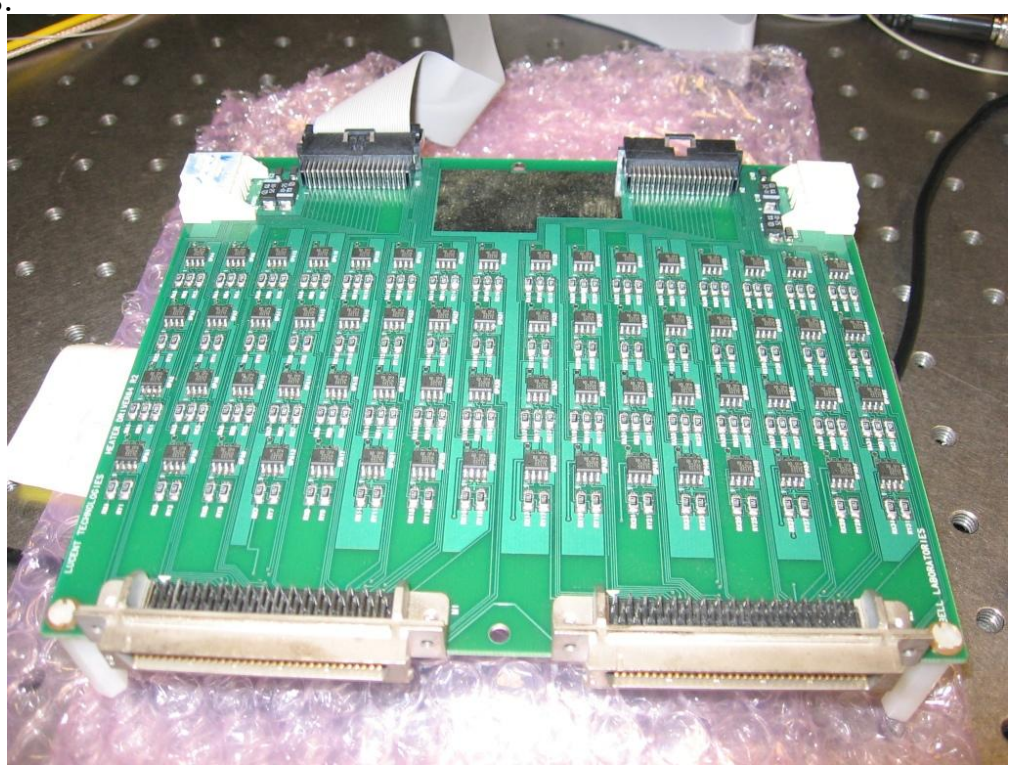


Figure 4.8: Photograph of National Instruments analog input/output card interface.

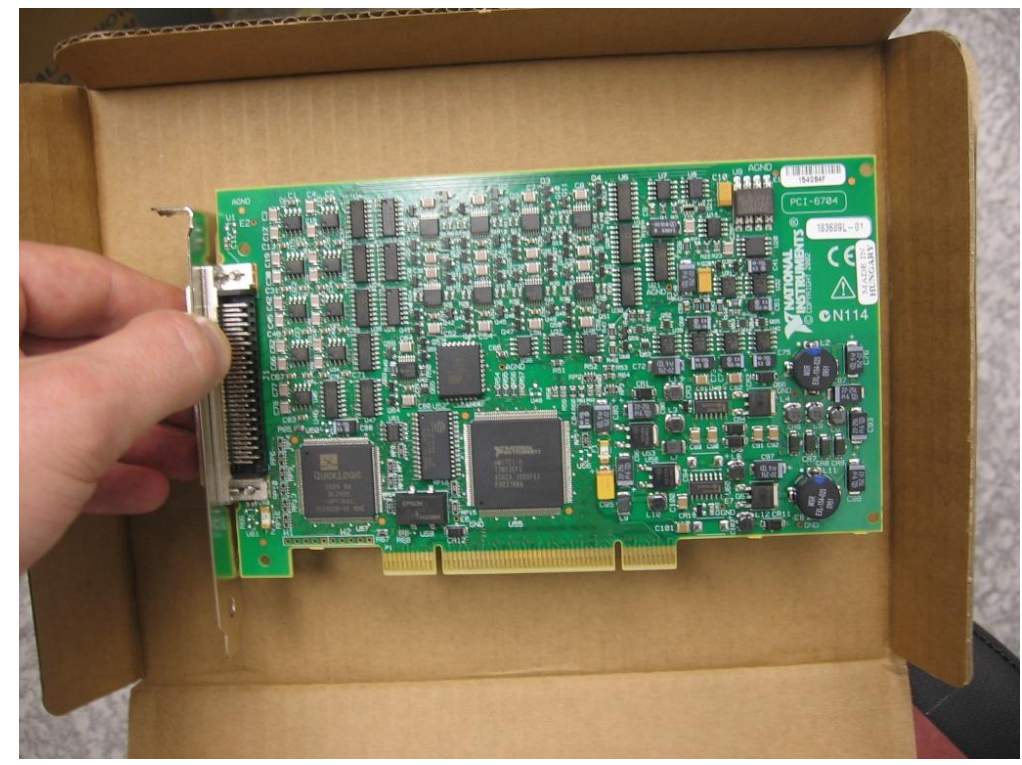

in principle choose all the parameters a-priori to implement desired filter coefficients. However, it is difficult to characterize precisely the correspondence between currents applied to each waveguide heater and the resulting optical phase shift. Imperfections in the filter fabrication also make the characterization difficult. Therefore, feedback is used to choose the correct biases to the heaters.

We use an optimization routine employing a downhill-simplex algorithm to tune the heater settings for the discriminator filter. Two radio frequency synthesizers are used to generate tones at $2 \mathrm{GHz}$ and $2.0001 \mathrm{GHz}$ with equal RF powers. The error signal for the optimization routine is the third-order intermodulation distortion term at $1.9999 \mathrm{GHz}$, normalized to the dc photocurrent and the fundamental signal power. The start point for each heater is randomly chosen within an acceptable range of currents which will not cause damage to the device. The routine varies the heater settings to minimize the error signal, thus maximizing the OIP3. The routine reaches a minimum error value after less than 100 iterations.

One of the filters was tuned to the desired linear ramp and linear phase transfer function. The phase and amplitude of the filter were measured with an optical vector network analyzer (OVNA). The transfer function shown in Fig. 4.9 is normalized to a $7 \mathrm{~dB}$ filter insertion loss. The insertion loss could be improved by better fiber coupling into the filter. The waveguide loss for silica PLC is not a significant loss mechanism. At the 50\% field amplitude transmission point, both the amplitude and phase of the transfer function appear linear within the accuracy of the instrument.

We report distortion measurements made with a single branch of the filter and single-ended detection. With tones at $2 \mathrm{GHz}$ and $2 \mathrm{GHz}+100 \mathrm{kHz}$ for the fundamental frequencies, we stepped the wavelength of the laser to determine the optimal bias point on the filter. At each wavelength, we collected the receiver power at $2 \mathrm{GHz}$ and the third-order intermodulation distortion (IMD3) power at $2 \mathrm{GHz}-100 \mathrm{kHz}$. 
Figure 4.9: Achieved filter amplitude and phase for the 6th order FIR lattice filter.

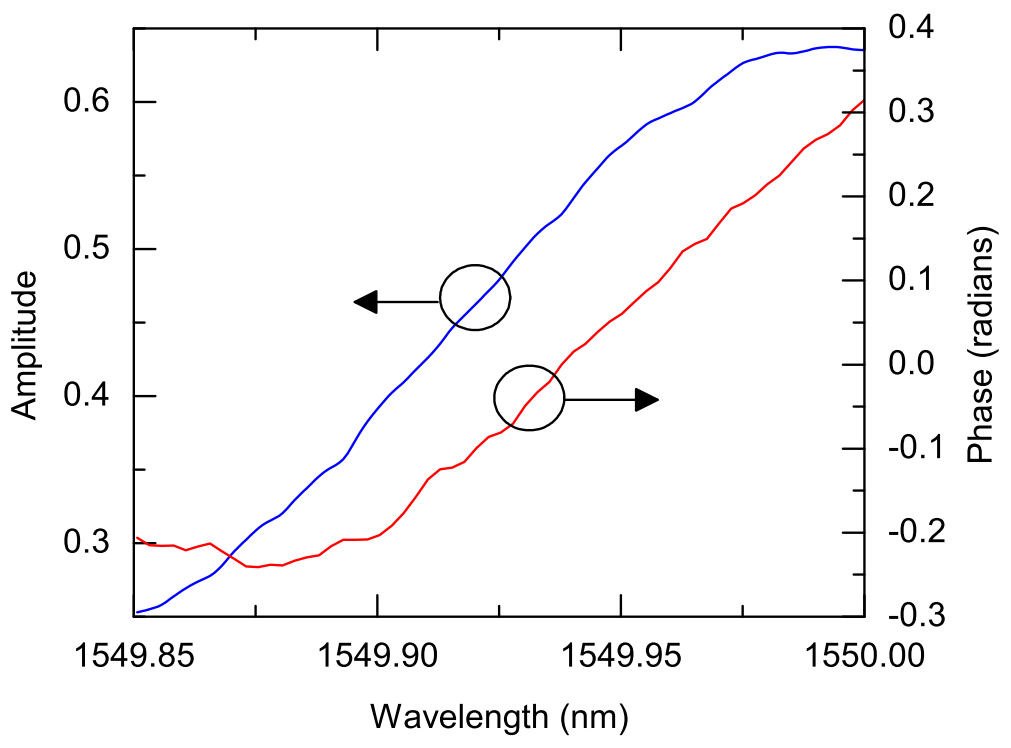

Fig. 4.10 shows the fundamental power and third-order nonlinearity as a function of carrier wavelength. The distortion remains low over a wavelength span of $10 \mathrm{pm}$. A $1549.937 \mathrm{~nm}$ carrier wavelength gives an optimal ratio of fundamental to IMD3 power. The optimal operating wavelength corresponded with a point where the filter has $50 \%$ amplitude transmission. For a system with a fixed wavelength of operation, the filter itself can be tuned in wavelength by adjusting the phase delays in each stage. A single filter could also be tuned to accommodate a variety of sources at different wavelengths, such as wavelength division multiplexing channels, since the filter transfer function repeats over each free-spectral range.

At the optimal wavelength, we varied the RF power input into the link and measured the IMD3 and fundamental power. The data is shown in Fig. 4.11. The distortion clearly showed a cubic dependence with input power. For a photocurrent of $0.11 \mathrm{~mA}$, we measured an OIP3 of $-19.5 \mathrm{dBm}$. The OIP3 of a PM link using an MZI discriminator and the same photocurrent is $-26.2 \mathrm{dBm}$. This particular transfer function displayed a $6.7 \mathrm{~dB}$ OIP3 performance improvement over an MZI with the samed received photocurrent. For shot-noise limited noise performance, the link has a spurious free dynamic range of $112 \mathrm{~dB} \cdot \mathrm{Hz}^{2 / 3}$. If the light is amplified to produce 10 $\mathrm{mA}$ of photocurrent, OIP3 increases to $19.7 \mathrm{dBm}$ and the shot-noise limited spurious free dynamic range is $125 \mathrm{~dB} \cdot \mathrm{Hz}^{2 / 3}$.

These initial results using optical lattice filters to discriminate phase modulation show appreciable improvement in third-order nonlinearity over the MZI, achieving 6.7 $\mathrm{dB}$ improvement in OIP3 at $2 \mathrm{GHz}$ modulation frequency, for a $10 \mathrm{pm}(1.25 \mathrm{GHz})$ span of carrier wavelengths. Because electrical spectrum monitoring and a feedback algorithm are used to optimize the filter coefficients, the system is robust to fabrication imperfections and other variations in the devices. Our particular FIR lattice filter had variations in switch contrast and waveguide loss that were corrected by the optimization. No assumptions were made about the mapping of the heater settings 
Figure 4.10: Fundamental and third-order intermodulation distortion versus laser wavelength. The modulation power is fixed at $10 \mathrm{dBm}$ and the photocurrent is fixed at $0.11 \mathrm{~mA}$.

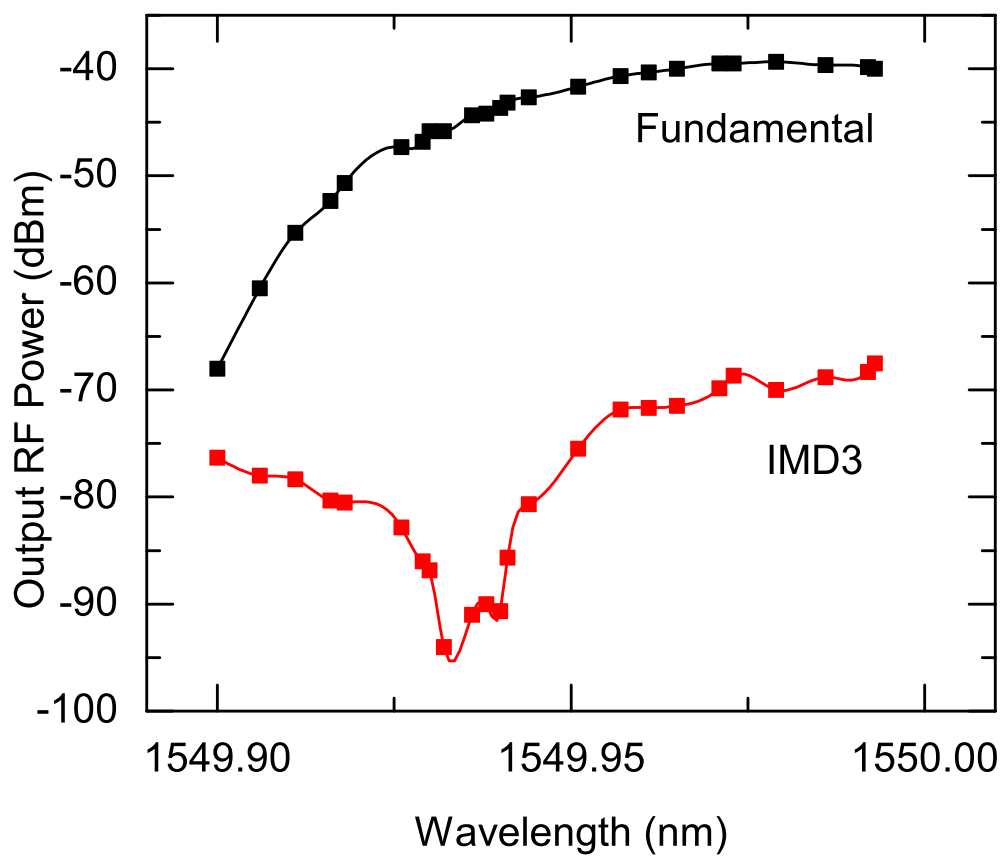

Figure 4.11: Fundamental and third-order intermodulation distortion versus modulation power. The photocurrent is fixed at $0.11 \mathrm{~mA}$ and the wavelength is fixed at $1593.7 \mathrm{~nm}$.

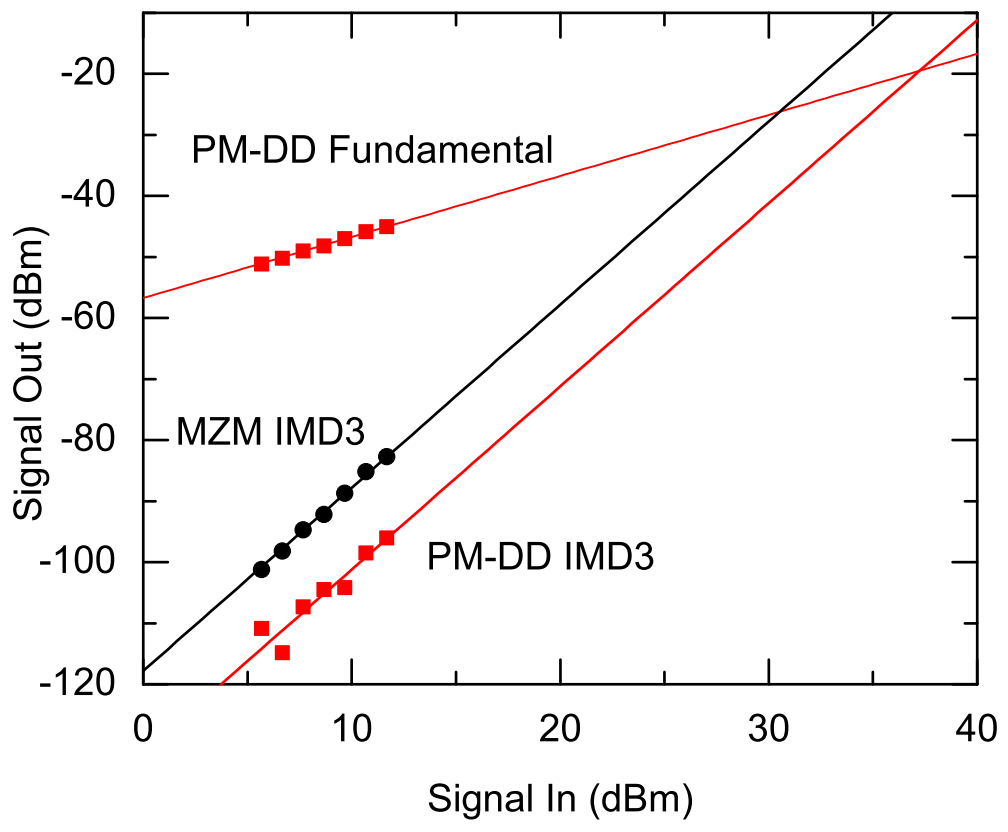


Figure 4.12: Achieved filter amplitude and phase for the RAMZI filter.

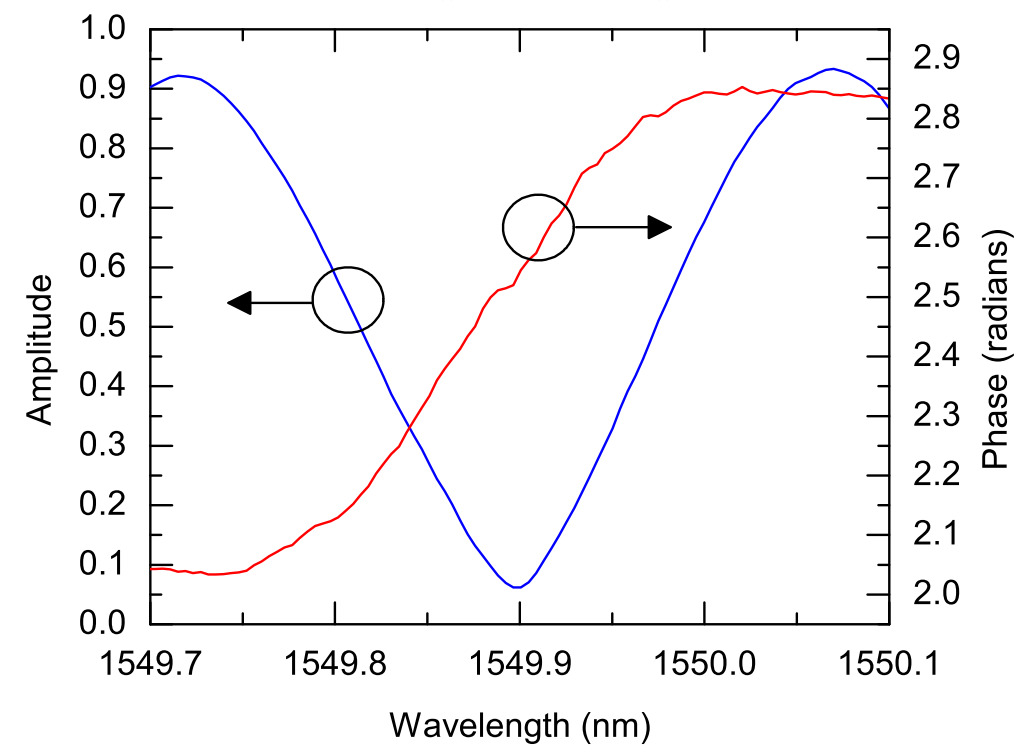

for the filter to its transfer function to run the optimization routine, so the technique could be used for any optical filter architecture. Theory and simulation suggest that much larger improvements in linearity are possible through careful adjustment of the filter transfer function.

Besides just further improvement in nonlinearity, the optimization goal can also be changed to seek improvement in signal gain, or nonlinearity improvement over chosen carrier wavelengths or modulation frequency spans. High-order filters could also be optimized to correct for gain equalization and fiber dispersion. For these reasons, using general purpose, tunable filters for phase discrimination is a very promising technique and suitable for much further study.

\subsubsection{Phase-modulated link with IIR filter}

We also performed PM-DD measurements with the IIR filter. Fig. 4.12 shows the tuned transfer function of one of the branches of the RAMZI filter. The transfer function was tuned by hand using the OVNA measurement for reference. The plotted transfer function is normalized to $5 \mathrm{~dB}$ insertion loss. The transfer function was measured with an OVNA. The second branch was tuned to a transfer function with opposite slope.

For our first distortion measurement, we use fundamental frequencies of $5 \mathrm{GHz}$ and $5 \mathrm{GHz}+100 \mathrm{kHz}$. Like with the FIR filter, we stepped the wavelength of the tunable laser and measured the fundamental and IMD3, but in this case, the measurement is performed with the balanced detection and two filters. The optical power is amplified so that the total DC photocurrent added from the two detectors is $10.5 \mathrm{~mA}$.. Fig. 4.13 shows the calculated OIP3 from the fundamental and IMD3 data. Also shown on the graph is the theoretical OIP3 from a dual-output MZM with the same DC photocurrent, which we exceed for a range of biases. It is important to note that 
Figure 4.13: Output intercept point of third-order intermodulation distortion versus laser wavelength in simulation and experiment. The total photocurrent is fixed at $10.5 \mathrm{~mA}$ and the modulation frequency is $5 \mathrm{GHz}$. The theoretical OIP3 of a link with a dual-output MZM and the same received photocurrent is also plotted in the figure.

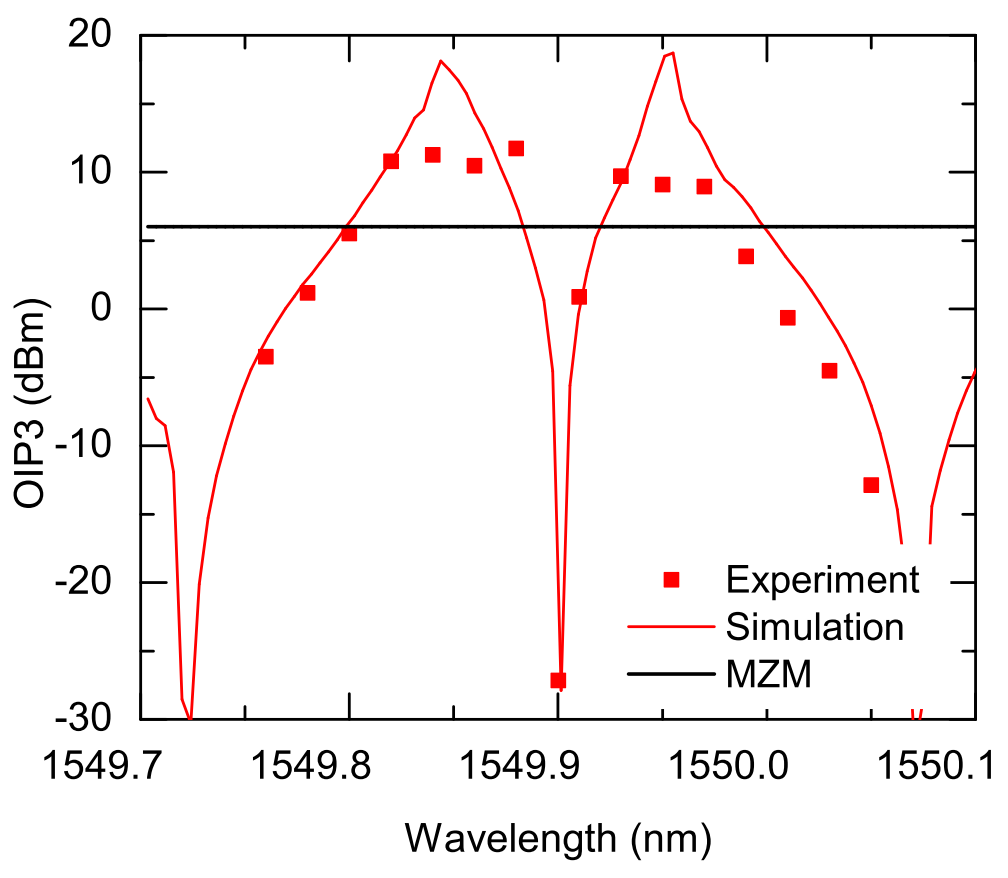

the measured and theoretical power values include a $6 \mathrm{~dB}$ power loss due impedance matching for the photodetector

Furthermore, the measured OVNA transfer function was used in a numerical link simulation, as described in Chapter 3. The simulation trend of OIP3 versus wavelength matched with the experimental data, except for peaks around the optimal bias points. This may be due to optical system factors not included in the simulation, such as balancing the two filters, fiber dispersion, polarization drift, back reflections, etc., or may be due to lack of resolution of the OVNA in measuring ripples in the transfer function.

We tuned the wavelength to $1549.964 \mathrm{~nm}$, which is approximately $50 \%$ field transmission bias and around the maximum measured OIP3 at $5 \mathrm{GHz}$. Varying the modulation frequency, we measured both the IMD3 and the second harmonics, and calculated OIP3 and OIP2, shown in Fig. 4.14. For frequencies between 1 to $10 \mathrm{GHz}$, we exceed the OIP3 of the MZM. The best frequency was around $4 \mathrm{GHz}$, which gave us an 8.5 dB OIP3 improvement over the dual-output Mach-Zehnder. Because it is a balanced device, the second-order distortion is also low over the whole band. The link gain increases for higher frequencies because of the $1 / \mathrm{f}$ PM to FM correspondence.

We compared the PM link and a dual-output MZM link using a high-power balanced photodetector array in development [90]. As shown in Fig. 4.15, the PM-DD link achieved $4.3 \mathrm{~dB}$ better RF compression power than the dual MZM. The link is nearly transparent, with only $3.8 \mathrm{~dB}$ signal loss. As shown in Fig. 4.16, increasing 
Figure 4.14: OIP3 and OIP2 versus modulation frequency at a fixed photocurrent of $10.5 \mathrm{~mA}$ and wavelength of $1549.964 \mathrm{~nm}$.

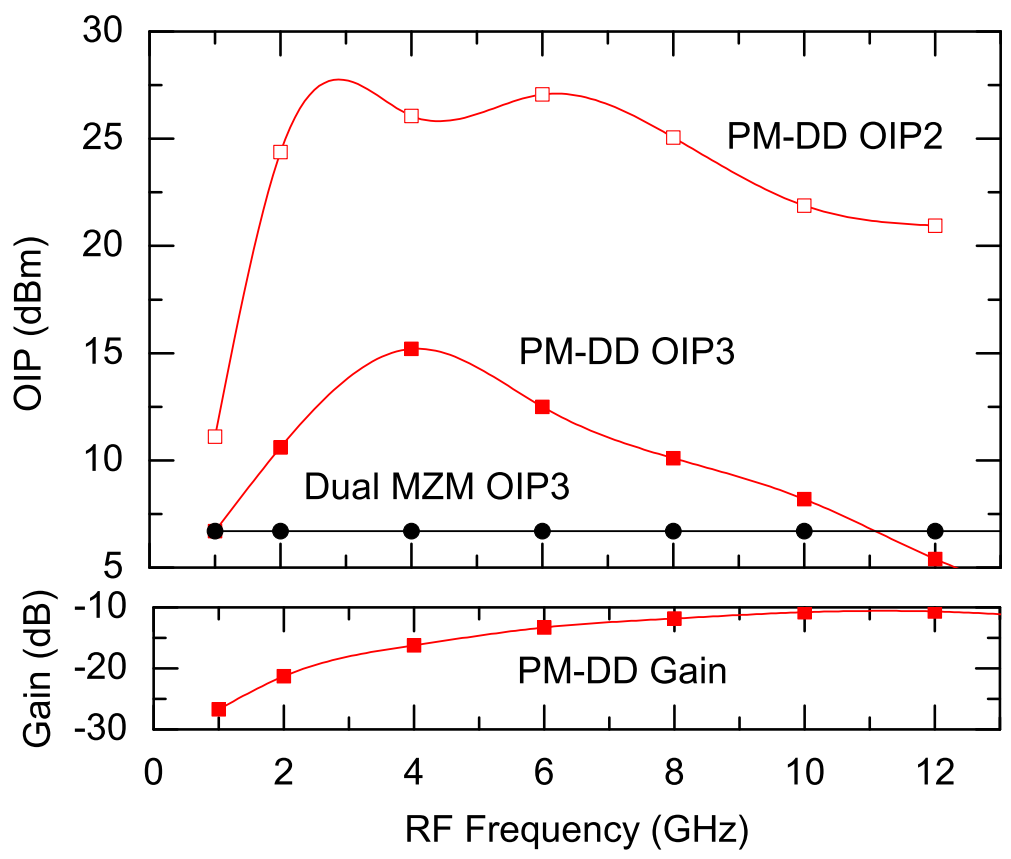

the effective photocurrent, we achieved a maximum OIP3 of $39.2 \mathrm{dBm}$ at $4 \mathrm{GHz}$ modulation frequency.

\subsection{Summary}

In this chapter, I have reviewed previous work in implementing FIR filters in photonics using optical lattice filters, and discussed experimental results using phase modulation. Using a sixth order optical lattice filter and a feedback optimization system, we instantiated a linear-field and linear-phase filter transfer function. This gave a $6.7 \mathrm{~dB}$ improved OIP3 over an MZI for a $2 \mathrm{GHz}$ phase modulated link. Using a 3rd-order IIR filter, we demonstrated improved OIP3 over an MZI for frequencies between 1 to $10 \mathrm{GHz}$, with the optimal improvement of $8.5 \mathrm{~dB}$ at frequency $4 \mathrm{GHz}$. Using high optical power, we demonstrate an OIP3 of $39.2 \mathrm{dBm}$. The link had low second-order distortion when using balanced detection. 
Figure 4.15: Output power versus modulation power compared to a dual-output Mach-Zehdner modulator measured experimentally. The frequency is fixed at 3.3 $\mathrm{GHz}$ and the effective DC photocurrent at $141 \mathrm{~mA}$.

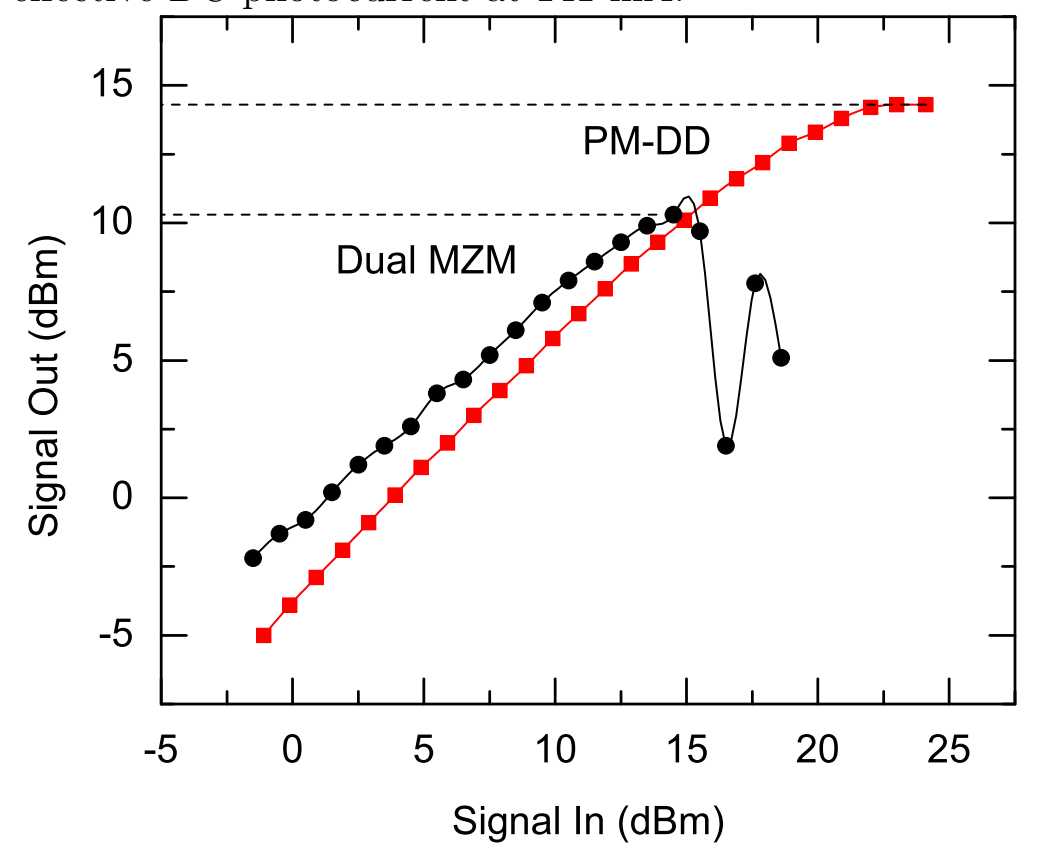

Figure 4.16: OIP3 versus effective DC photocurrent. The frequency is fixed at 4.0 $\mathrm{GHz}$ and the modulation power at $0 \mathrm{dBm}$.

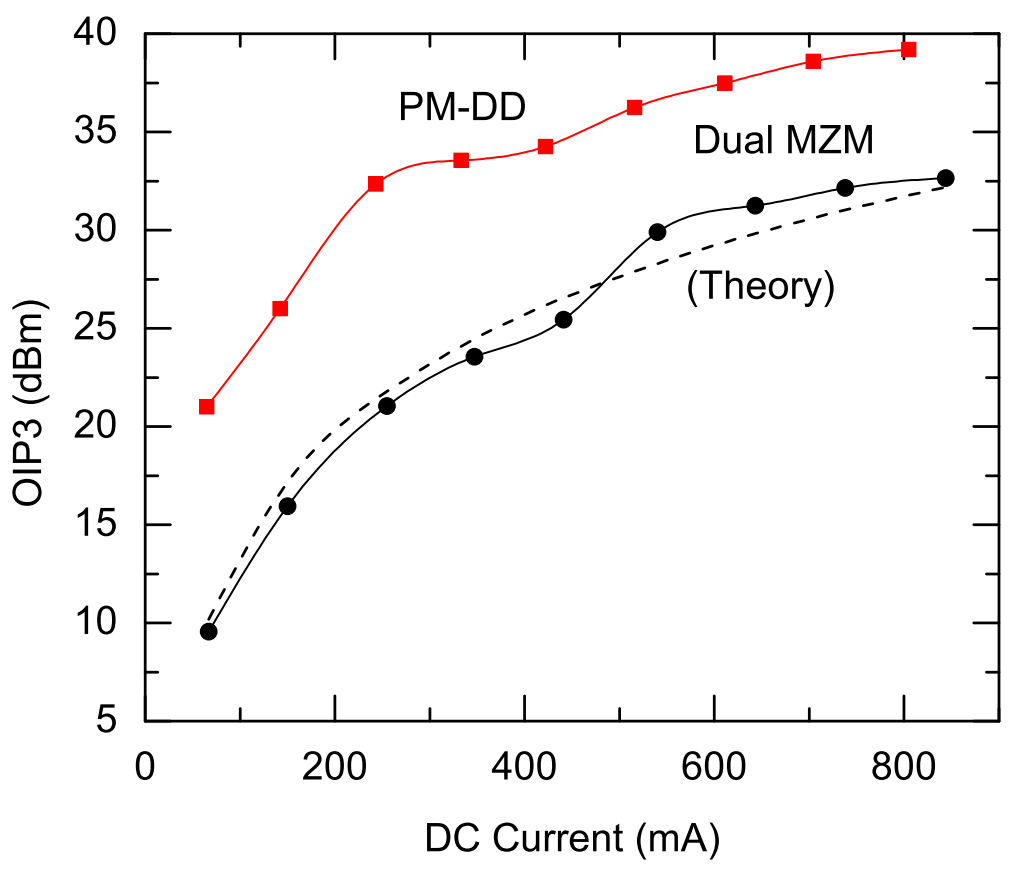




\section{Chapter 5}

\section{Frequency modulation experiments}

In this chapter, I present a review of work in the field of FM lasers, the experimental characterization of current modulated, distributed Bragg reflector lasers developed for this program of research, and microwave photonic link results using the FM lasers and PLC discriminator filters.

\subsection{Review of FM lasers}

There has been much work over the years in designing frequency modulated lasers. For application in microwave photonic links, the theory in Chapter 2 indicates that the FM lasers must have small linewidths $(<100 \mathrm{kHz})$ and large modulation efficiency (of the order of $10 \mathrm{~s}$ of $\mathrm{GHz} / \mathrm{mA}$ or $100 \mathrm{~s}$ of $\mathrm{GHz} / \mathrm{V}$ ) in order to give links with small noise figures. In addition, the lasers also must have low residual intensity modulation (Section 2.6.3) and low intrinsic distortion in order to ensure linearity. Frequency modulation can be produced in semiconductor lasers by active gain modulation, phase modulation, or grating modulation of single or multi-section devices. Both distributed Bragg reflector (DBR) and distributed feedback (DFB) lasers have been used in frequency modulation experiments. The phase shift in each section is either produced by current or voltage modulation. In current modulation, the junction is forward biased, and injected carriers change the refractive index through the free-carrier plasma effect. In voltage modulation, the junction is reverse biased, and a field effect such as the Franz-Keldysh (FK) effect in bulk material or the quantumconfined Stark effect (QCSE) in quantum-well material produces a refractive index change. For a low efficiency approach to frequency modulation, [91] and [92, 93] have suggested using phase modulators operated past their RC limited bandwidth.

There have been a number of theoretical papers looking at laser distortion under frequency modulation. [94-96] used Bessel function and perturbation models to solve for harmonic and intermodulation distortion in laser IM and FM. In [48, 97-102], the authors used Taylor-series analyses to estimate the harmonic and intermodulation distortion of an FM laser after a Mach Zehnder interferometer. Unfortunately, none of these papers applied their theory to multi-section devices. 


\subsubsection{Fabry-Perot lasers}

In some of the earliest work on frequency-modulation of semiconductor lasers, [42] measured the direct FM and IM characteristics of three single-section Fabry-Perot AlGaAs lasers. The results were consistent with models for thermal and carrier injection effects. The device response not flat and decreased several orders of magnitude from low to high modulation frequency. Modulation efficiencies were of the order of 1 $\mathrm{GHz} / \mathrm{mA}$ for low frequencies (thermal), and $100 \mathrm{MHz} / \mathrm{mA}$ for high frequencies (carrier injection), which is not sufficient for microwave photonic links. The nonlinearity of the modulation was not studied.

Later Fabry-Perot devices used a second tuning section and QCSE based voltage modulation to increase the modulation bandwidth. The device reported in [103] had a flat response up to $2 \mathrm{GHz}$, frequency modulation efficiency of $7 \mathrm{GHz} / \mathrm{V}$, and 3.7 MHz minimum linewidth. [104] claimed a simpler fabrication technique for the same type of device. Their improved laser had flat, RC limited response from 5 $\mathrm{kHz}$ to $500 \mathrm{MHz}$, with a modulation efficiency of $20 \mathrm{GHz} / \mathrm{V}$. In addition, the laser also had very low residual IM. The residual intensity modulation depth was 0.05 for a frequency deviation of $4 \mathrm{GHz}$. To demonstrate the benefit of the reverse biased, QCSE modulation, measurements were compared with forward biased carrier injection. The efficiency ranged from $1.7 \mathrm{GHz} / \mathrm{mA}$ at $5 \mathrm{kHz}$ to $0.3 \mathrm{GHz} / \mathrm{mA}$ at $50 \mathrm{MHz}$. In a later device, [105], the capacitance was improved, thus giving a uniform FM response from $30 \mathrm{kHz}$ to $6 \mathrm{GHz}$, and FM efficiency of about $10 \mathrm{GHz} / \mathrm{V}$. The linewidth was approximately $10 \mathrm{MHz}$. The residual IM was low for this device: the IM depth was 0.05 for a frequency deviation of $4 \mathrm{GHz}$. For this device under forward bias, the FM efficiency was $1 \mathrm{GHz} / \mathrm{mA}$ at low frequency but fell off rapidy to $10 \mathrm{MHz} / \mathrm{mA}$ at 1 $\mathrm{GHz}$. The residual IM depth was 0.09 for frequency deviation of $3.8 \mathrm{GHz}$.

\subsubsection{DBR lasers}

Multi-section distributed Bragg reflector lasers have also been explored. In [106], the authors presented a two section DBR laser with grating section modulation and current injection. The modulation bandwidth was $400 \mathrm{MHz}$ and the modulation efficiency was $3 \mathrm{GHz} / \mathrm{mA}$. The linewidth was approximately $50 \mathrm{MHz}$. For $1 \mathrm{GHz}$ frequency deviation, the RIM depth was 0.01. Another author, [107], used a multi-electrode DBR laser to produce pure frequency modulation. Because carrier injection in a passive grating section has limited bandwidth, they instead modulated the active region of the laser with a non uniform current density. With only single electrode modulation, the bandwidth was $600 \mathrm{MHz}$, the modulation efficiency was $200 \mathrm{MHz} / \mathrm{mA}$, and the IM efficiency was $0.014 / \mathrm{mA}$. The bandwidth was limited by the capacitance of the device. With push-pull, dual electrode modulation, the FM efficiency was $360 \mathrm{MHz} / \mathrm{mA}$ and IM efficiency was $0.00252 / \mathrm{mA}$. In [108], a $5 \mathrm{MHz}$ linewidth was reported for the same device. [109] carried out a theoretical analysis of current injected, three-section (gain, phase, grating) DBR lasers using general rate equations. The authors solved for the frequency dependence of the FM and IM modulation efficiencies.

Later DBR devices used voltage modulation. The laser in [110] used a MQW gain 
section and superlattice passive sections. The authors measured a $4 \mathrm{GHz}$ bandwidth, with $38.75 \mathrm{GHz} / \mathrm{V}$ modulation efficiency. A similar device was reported by [111, 112], which used the Franz-Keldysh effect to tune the Bragg section. The linewidth was less than $10 \mathrm{MHz}$ over the tuning range. The authors published another device using an electro-absorption modulated section in $[113,114]$. [115] theoretically studied how to optimize phase-section modulated QCSE DBR lasers. The theoretical modulation efficiency was $2.5 \mathrm{GHz} / \mathrm{V}$, and the spurious intensity modulation was suppessed. More recently, [116] presented a three-section InGaAsP DBR laser. In this device, the passive and active use the same MQW material, but the absorption was reduced in the active sections by using quantum-well intermixing (QWI) based on ion implantation. The modulation response was uniform from $10 \mathrm{MHz}$ to $10 \mathrm{GHz}$. The tuning efficiency of the DBR section was $375 \mathrm{GHz} / \mathrm{V}$ at dc. In $[117,118]$, the authors modulated the phase-section of a DBR laser. To reduce residual IM and increase the bandwidth, the authors designed the bandgap of the phase control region to be much wider than that of the gain region, so the waveguide loss variation induced by the refractive index modulation would be extremely small. The authors presented an array of four lasers in [119]. The modulation efficiency was approximately $5 \mathrm{GHz} / \mathrm{V}$. The linewidth of the lasers was $15 \mathrm{MHz}$.

\subsubsection{DFB lasers}

Finally, authors have studied FM distributed feedback lasers with single and multiple electrodes. [120] measured the modulation response versus frequency of a commerical 1.3 micron DFB laser. The bandwidth was approximately $4.3 \mathrm{GHz}$, but it was not flat, there was an observeable resonance peak. At $1 \mathrm{GHz}$, the FM response was 170 $\mathrm{MHz} / \mathrm{mA}$, and the IM response was $0.022 / \mathrm{mA}$. [121] developed a two section DFB laser, with a DFB active region and a phase tuning region. The phase region was modulated with $200 \mathrm{MHz}$ bandwidth. The maximum FM efficiency at $10 \mathrm{MHz}$ was $16 \mathrm{GHz} / \mathrm{mA}$. The linewidth was of the order of $50 \mathrm{MHz}$. The intensity modulation was less than 0.01 for $1 \mathrm{GHz}$ frequency deviation. [122] reported a complex coupled DFB laser with a specially engineered grating. It had a flat FM response from $10 \mathrm{kHz}$ to more than $20 \mathrm{GHz}$, limited by the measurement system. The modulation efficiency was $0.95 \mathrm{GHz} / \mathrm{mA}$ at $1 \mathrm{GHz}$, and the linewidth was $12 \mathrm{MHz}$.

$[29,123]$ gave a theoretical model for a two section DFB laser using a QCSE phase section. In the results, the highest FM efficiency reported was $80 \mathrm{GHz} / \mathrm{V}$ at about $3 \mathrm{GHz}$ modulation. The modulation was not flat, but the bandwidth was greater than several GHz. The authors argued that DBR lasers are better than DFB lasers because the phase-modulating section can occupy a relatively larger fraction of the cavity leading to higher FM efficiency.

[124] presented a multi-electrode DFB device. Multiple electrodes were engineered so that carrier and thermal effects would be in phase. There were electrodes on the center and sides of the active DFB region. The modulation efficiency was flat from 100 $\mathrm{kHz}$ to more than $15 \mathrm{GHz}$. At $100 \mathrm{kHz}$, the modulation efficiency was $0.64 \mathrm{GHz} / \mathrm{mA}$. At $1 \mathrm{GHz}$, it was $0.37 \mathrm{GHz} / \mathrm{mA}$. The authors used a long $1.2 \mathrm{~mm}$ cavity to reduce the linewidth to less than $1 \mathrm{MHz}$. [125] reported a DFB laser with 5 electrodes. The 
Figure 5.1: Self heterodyne laser linewidth measurement experimental setup.

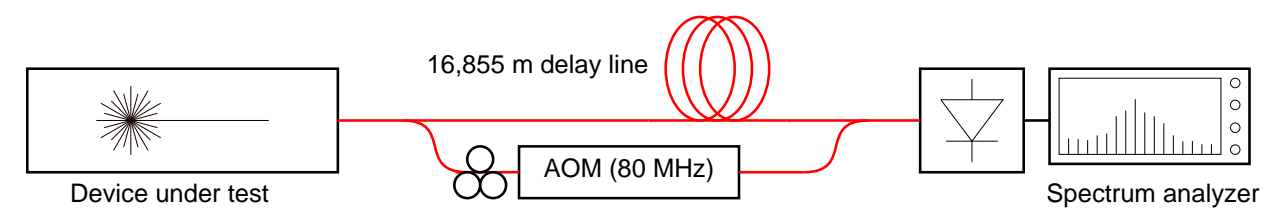

bandwidth was $4 \mathrm{GHz}$, the FM efficiency was $0.65 \mathrm{GHz} / \mathrm{mA}$, and the linewidth was $3 \mathrm{MHz}$.

$[30,126,127]$ reported a high efficiency FM laser using an integrated DFB laser and electroabsorption modulator. The modulator facet was not AR coated so the reflectivity from the facet contributes to the phase change. The bandwidth was about $8 \mathrm{GHz}$ and the modulation efficiency approximately $10 \mathrm{GHz} / \mathrm{mA}$. The linewidth was $60 \mathrm{MHz}$.

A number of the Fabry-Perot, DBR, and DFB FM lasers that have been reported have very high modulation efficiency. This is very desirable for microwave photonic links to ensure low RF signal loss or even link gain. However, the devices with the highest efficiency also had some of the worst linewidths. None of the linewidths reported were less than $1 \mathrm{MHz}$. Additional work still needs to be done to optimize FM semiconductor lasers for both low linewidth and high modulation efficiency to produce links with low noise figures and high dynamic range. In addition, theoretical and experimental work needs to be performed to find out the limitations of the modulation nonlinearities of multi-section FM lasers.

\subsection{Laser characterization}

The FM lasers we had available for our experiments were three-section (gain, phase, grating) and four-section (gain, phase, grating, phase) distributed Bragg reflector lasers designed by Bell Laboratories and fabricated by Multiplex Inc. The devices were optimized for high modulation efficiency, low linewidth, and low residual intensity modulation.

I performed a self-heterodyne measurement to characterize the linewidth of two of the FM lasers. This technique was developed by [128], and consists of heterodyning the laser with a time-delayed and AOM frequency shifted version of itself. For a Lorentzian lineshape, the beat tone has a linewidth that is twice the laser's linewidth. Fig. 5.1 shows the experimental setup of the self-heterodyne measurement. Fig. 5.2 shows the $80 \mathrm{MHz}$ beat-tones from the experiment. Each laser's gain section was biased at $200 \mathrm{~mA}$ at a temperature of $20 \mathrm{C}$. The three-section laser had approximately a $174 \mathrm{kHz}$ linewidth, and the four-section laser had a $161 \mathrm{kHz}$ linewidth.

I performed a DC tuning measurement to choose optimal bias points for modulation. The lasers were gain biased at $200 \mathrm{~mA}$, and the phase section bias was adjusted. The wavelength was viewed on a high-resolution optical spectrum analyzer. Fig. 5.3 shows the tuning curves for the two devices. At $1.3 \mathrm{~mA}$ phase section bias, 
Figure 5.2: Self heterodyne laser spectrum measurements with Lorentzian fits.
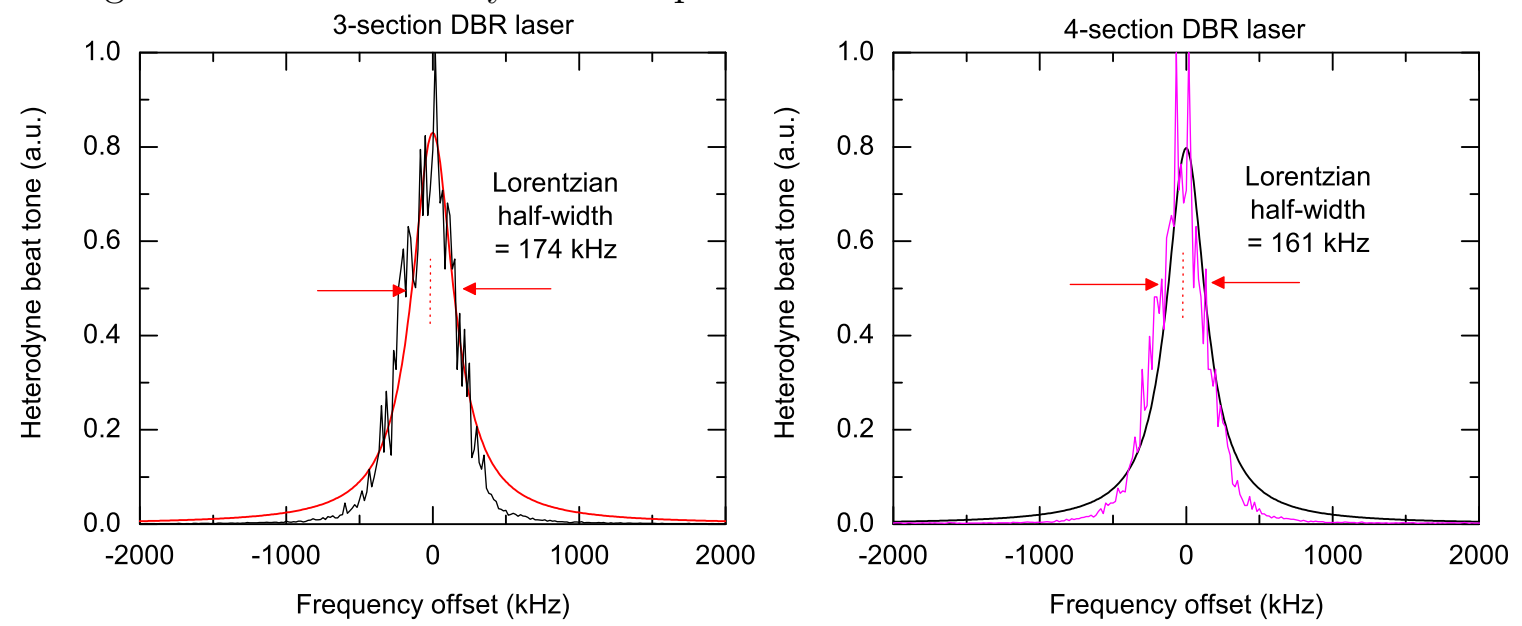

the DC tuning slope for the 3-section laser was $22 \mathrm{GHz} / \mathrm{mA}$, and the tuning slope for the 4 -section laser was $9 \mathrm{GHz} / \mathrm{mA}$. At $0.5 \mathrm{~mA}$ phase section bias, the DC tuning slope for the 3 -section laser was $45 \mathrm{GHz} / \mathrm{mA}$. The 3 -section device had a much higher efficiency.

RF modulation efficiency measurements were performed with a vector network analyzer. Fig. 5.4 shows the experimental setup. A 50 GHz FSR (20 pS delay) MachZehnder interferometer was used to discriminate the FM. The modulation efficiency was calculated using the gain expression (5.1) corrected for the passive impedance matching in the experiment and corrected for reflected modulation power. The modulation efficiency for both devices at $10 \mathrm{MHz}$ was about $10 \%$ less than at DC. At 1.3 $\mathrm{mA}$ phase section bias, the bandwidth of the three-section laser was $70 \mathrm{MHz}$, and the bandwidth of the four-section laser was $75 \mathrm{MHz}$. At $0.5 \mathrm{~mA}$ phase section bias, the bandwidth of the three-section laser was $60 \mathrm{MHz}$. The effective FM modulation efficiency of a lithium niobate phase modulator is also shown on the plot. For frequencies less than $5 \mathrm{GHz}$, the link gain provided by the FM lasers is more than an order of magnitude better than that of the lithium niobate phase modulator.

Fig. 5.6 shows the expected phase-noise limited noise figure versus frequency. The calculation assumes a $50 \mathrm{ohm}$ input impedance, and smoothing has been applied to the graph. At $100 \mathrm{MHz}$ modulation frequency, the modulation efficiency of the 3section laser biased at $0.5 \mathrm{~mA}$ is high enough to provide a $6 \mathrm{~dB}$ noise figure, but the efficiency falls off rapidly.

$$
\eta=\frac{\left|S_{21}\right|}{i_{d c} 2 \pi \tau} \sqrt{\frac{\left|Z_{\text {in }}\right|}{\left(1-\left|S_{11}\right|^{2}\right)\left|Z_{\text {out }}\right|}}
$$

Finally, I characterized the residual intensity modulation of the three-section DBR laser biased at $1.3 \mathrm{~mA}$. A link S21 measurement was performed with the MZI to measure the FM response, and without the MZI, but at the same photocurrent, to measure the IM response. In both cases, only the phase-section of the device was modulated. At $100 \mathrm{MHz}$ modulation frequency, the IM was 27 dB lower than the FM. 
Figure 5.3: DC tuning measurement of DBR laser phase sections.

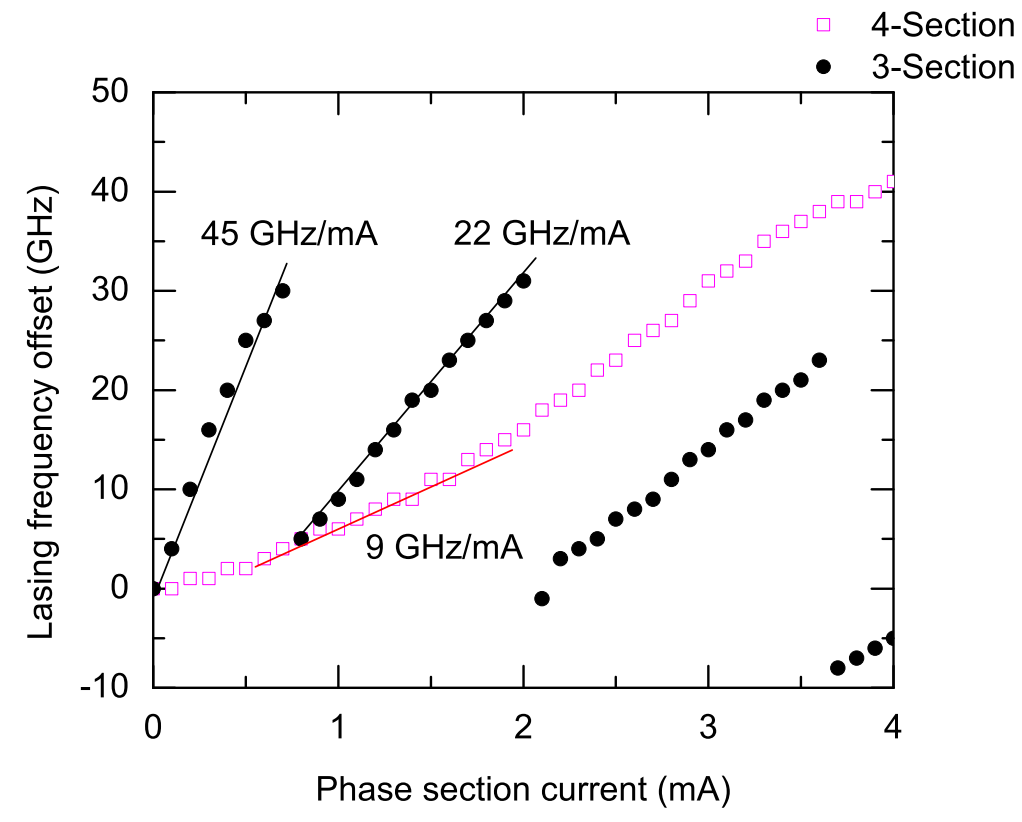

Figure 5.4: FM modulation efficiency experimental setup.

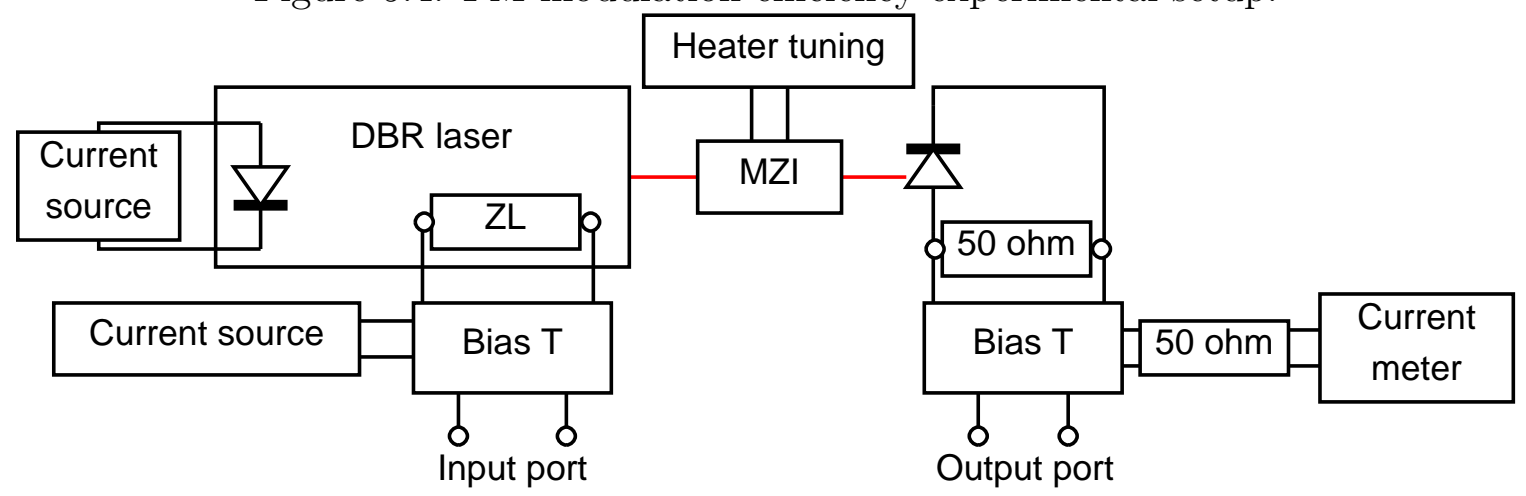


Figure 5.5: DBR FM modulation efficiency versus frequency.

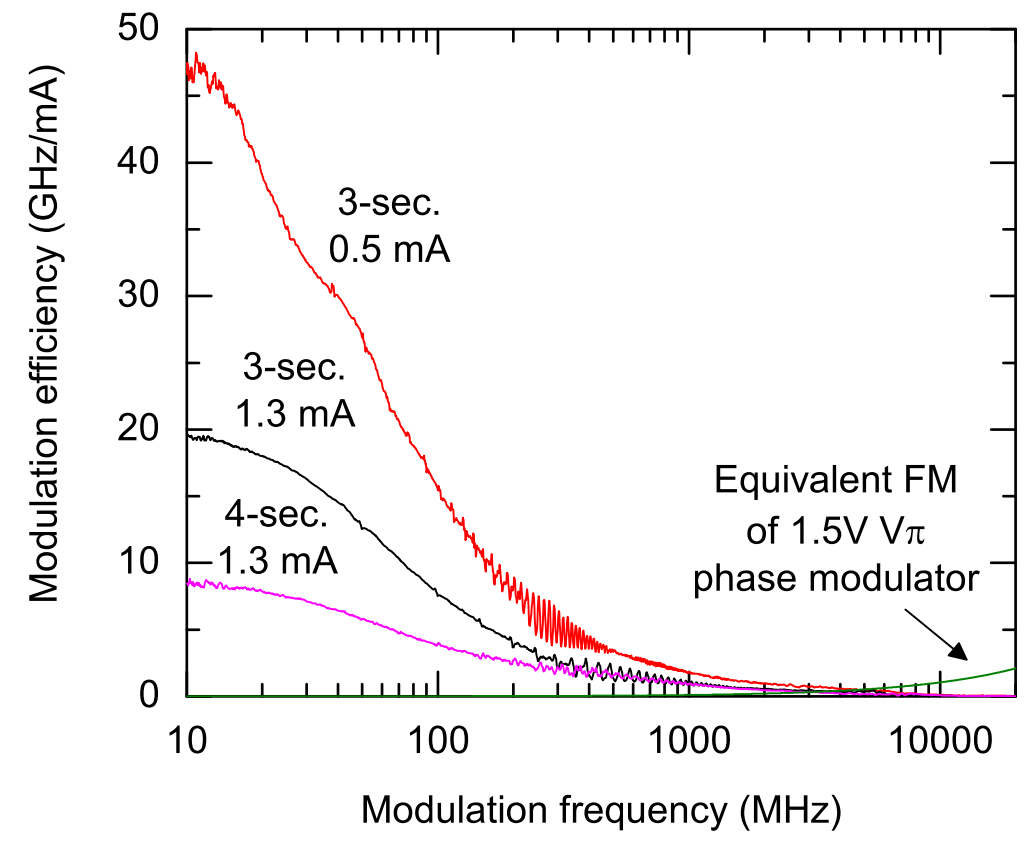

Figure 5.6: Phase-noise limited noise figure for FM DBR lasers from measured modulation efficiency and linewidth.

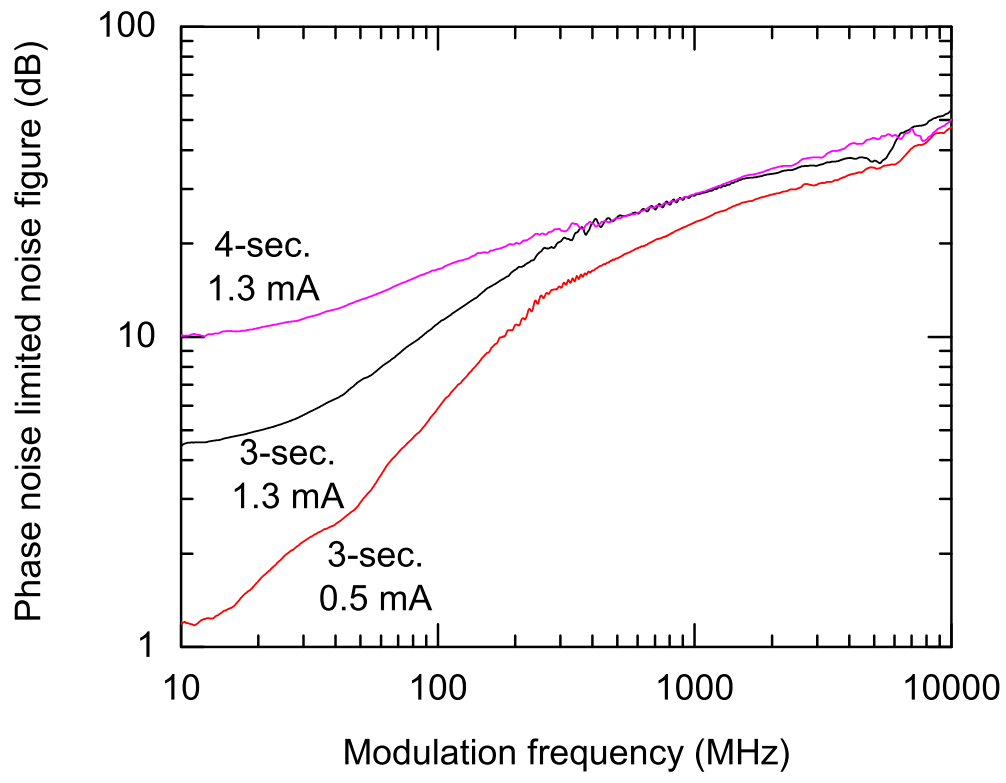


Figure 5.7: Residual intensity modulation measurement of DBR FM lasers.
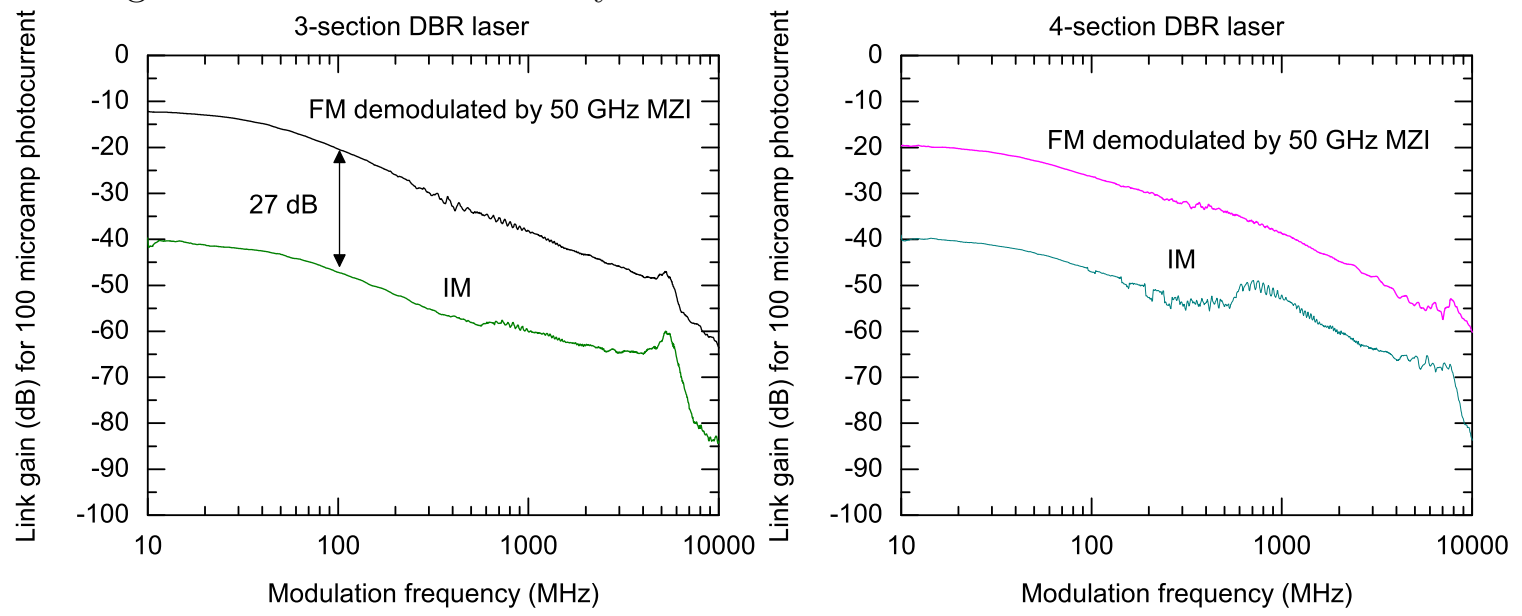

The residual IM was sufficiently small such that it would not degrade the linearity performance of the link.

Overall, the current-injected, 3-section DBR devices had high modulation efficiency, low linewidth, and low residual IM. However, they had very limited bandwidth. This is an intrinsic issue with current-injection, as the carrier lifetime is very long in passive phase-modulation sections. Additional work needs to be performed to develop voltage-modulated devices in order to increase the bandwidth of operation of the FM-DD microwave photonic links.

\subsection{Frequency-modulated link with IIR filter}

The FM laser used in the link-experiments is similar to the devices described in the previous section. It is a three-section DBR laser with a $45 \mathrm{kHz}$ linewidth and 7.5 $\mathrm{GHz} / \mathrm{mA}$ modulation efficiency at $100 \mathrm{MHz}$ by modulating the phase section of the device. The device was used in conjunction with the IIR discriminator to form an FM-DD link. Figure 5.8 on page 73 shows the gain of the link compared to the PM-DD link. For a fairly small photocurrent of $5.2 \mathrm{~mA}$ per detector $(10.5 \mathrm{~mA}$ total photocurrent), we are able to achieve positive link gain for the FM-DD link for frequencies up to $500 \mathrm{MHz}$. Although the modulation efficiency falls off fairly fast because the device is operated by current injection, the link gain exceeds the lithium niobate phase modulator up past $3 \mathrm{GHz}$.

We measured the distortion of the link and compare it to the PM-DD and IMDD. Figure 5.9 on page 73 compares OIP2 and OIP3 of the FM link with PM link data shown previously in Figure 4.14 on page 63 . At its most linear frequency the FM laser exceeds the OIP3 of the Mach Zehnder by $5.8 \mathrm{~dB}$, and the link has low second order distortion. The link gain and OIP3 degrade for higher modulation frequencies, but have significant improvements over IMDD and PM-DD links at low frequencies for both linearity and gain. 
Figure 5.8: Link gain versus modulation frequency for the FM link versus the PM+IIR link.

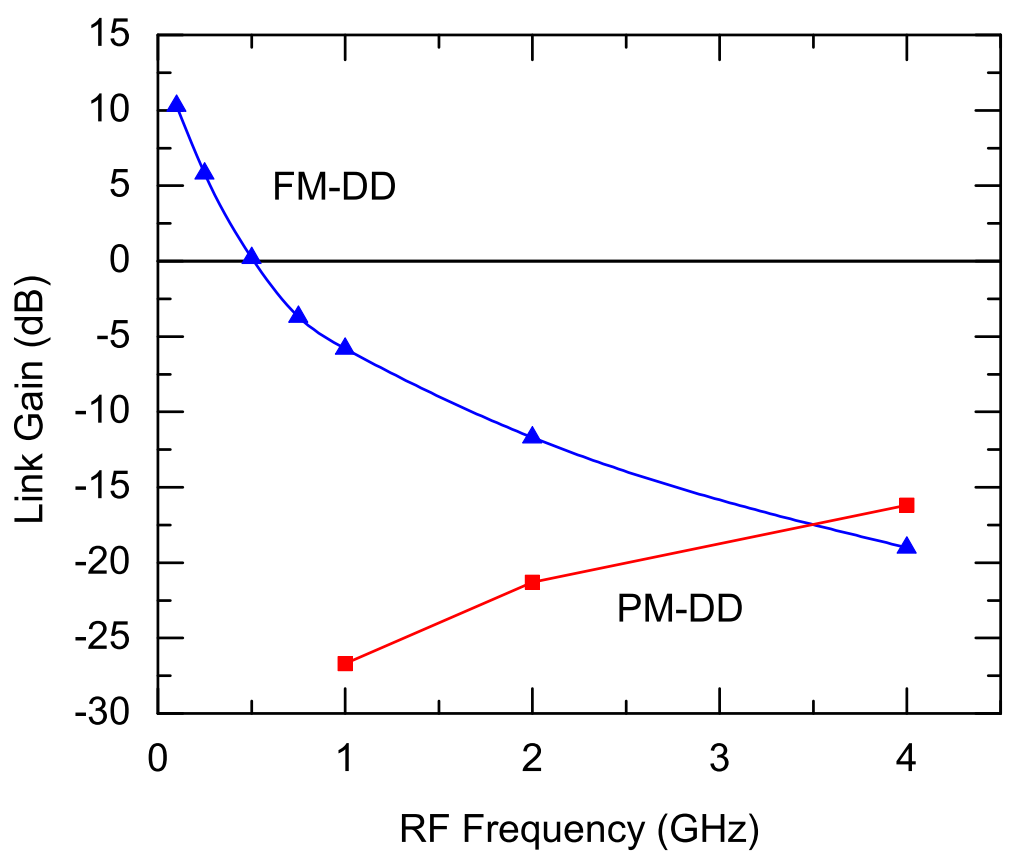

Figure 5.9: Distortion versus modulation frequency, compared to the results of the PM+IIR link.

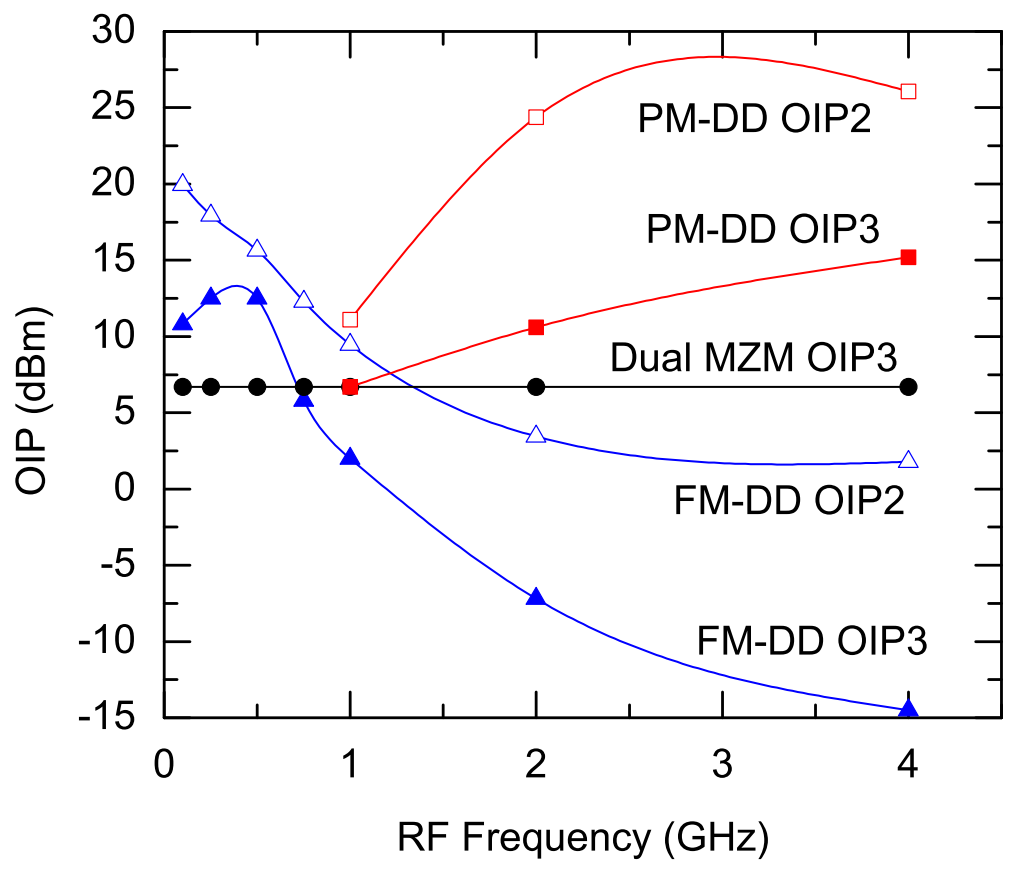




\subsection{Summary}

FM lasers are a promising transmitter for microwave photonic systems, mainly because of their very high modulation efficiency, which exceeds that of the best lithium niobate modulators by an order of magnitude. Previous work had not focused specifically on optimizing both laser linewidth and modulation efficiency in order to ensure low phase-noise limited noise figures when the links are operated with high optical power. We have demonstrated links that, within the laser's modulation bandwidth, provide noise figures $<10 \mathrm{~dB}$, and exceed the linearity of a MZM IM-DD link when using complementary, linear-field PLC filters for demodulation. With further optimization of the laser modulation bandwidth by using field-effect modulation rather than current-injection, the FM-DD link architecture should be able to provide microwave frequency links with high dynamic range, low noise figures, and signal gain. 


\section{Chapter 6}

\section{Conclusions and future work}

The work presented in this dissertation illustrates the benefits of phase-modulated and frequency-modulated microwave photonic links with direct detection (PM/FM-DD) as approaches to achieving microwave photonic systems with low noise-figure, high linearity, and high dynamic range. By fully modeling the nonlinear signal transmission in the links, we are able to accurately predict the performance of a link using particular optical filters in the demodulator. This dissertation has developed the tools necessary for validating the distortion produced by a particular filter designed to discriminate phase or frequency modulation. The close match between experimental results and simulation, as shown in Figure 4.10 on page 60, was very promising. The general large-signal and small-signal analytical expressions for filtered PM and FM links, as well as our numerical model, should be useful for future link designers.

On embarking on this program of research, it was to the surprise of this author that previous work had relied on heuristics and inaccurate Taylor-series models. Although PM/FM-DD has been actively explored since the 1960's, as discussed in Section 2.3, we were the first to present complimentary linear-field demodulation as a simple ideal model for which to design discriminator filters. Although the early work of [33] had suggested a linear-field transmission ramp, they did not identify the use of balanced detection to cancel second harmonics or provide any guidance about the phase linearity of the filter. Later authors tried to linearize the slope of the power transmission instead, which we show is misguided (Section 2.6). It is our hope that the models presented in this dissertation clear up remaining confusion.

Our experimental results showed appreciable improvements in the link distortion over Mach-Zehnder modulator IM-DD links. Intermodulation OIP3 was improved by over $6 \mathrm{~dB}$ over the MZM, and improvement was shown for frequencies up through 10 GHz. This research is a first step, and we believe we have not reached the full potential of this approach. Due to difficulties in controlling filter coefficients, we were not able to directly transfer designs from simulation to implementation. However, the reverse process, measuring the transfer function of a filter implementation and inserting it into simulation, provided accurate predictions of link performance. I would like to do additional analytical work on IIR flter design, and explore the scaling of achievable SFDR with IIR filter order. Fig 3.5. showed that we can achieve a $2 \mathrm{~dB}$ increase in 
SFDR per filter order scaling benefit from the FIR filter designs.

Due to their diversity of applications, there is comparably more work being performed in the field of microwave photonic filters than in linearization of microwave photonic links. The PM/FM-DD approach will benefit from synergies with that work. Another photonic-only approach to linear microwave photonic links, linearized intensity modulators, is not as general, since applicable fabrication processes are limited to those with optically active materials, such as lithium niobate. In PM-DD links, the modulation is simple and decoupled from the demodulation process. Passive filters may be easier to design and optimize as a separate component.

Frequency modulation of semiconductor lasers is another promising area of research. Links using FM directly benefit from PM-DD demodulator designs, with the added advantage of signal gain. There are a number of research topics to explore. Unanswered questions include theoretical models for and experimental characterization of the nonlinearity of the frequency modulation, and discovering the fundamental tradeoffs between noise, modulation efficiency, residual intensity modulation, and bandwidth for the semiconductor lasers. From my review of the literature, it is clear that this design space has not been fully explored. Chapter 5 shows that even with these unknowns, we were able to demonstrate a link with signal gain for a small photocurrent, gain exceeding a PM link up through $3 \mathrm{GHz}$ modulation frequency, and linearity exceeding that of an IM-DD MZM link, if only for a limited bandwidth.

A future area of research remains in the area of frequency up-converting and down-converting links. Frequency converting links, also called IF-to-RF or RF-toIF links, are important in many remote antenna systems. Electronic mixers produce large spurious signals, and photonic mixing is promising for reducing these conversion spurs. We are looking at methods to linearize and increase the dynamic range of these frequency-converting links by using planar-lightwave circuit filters. 


\section{Appendix A}

\section{Simulation code}

In order to design PM-DD and FM-DD microwave photonic systems, it is very important to accurately access tradeoffs between metrics such noise, linearity, bandwidth, power consumption, and cost. The following simulation code applies the theory developed in this dissertation towards modeling these complex systems.

\section{A.1 Small-signal simulation}

The small signal model for a PM-DD or FM-DD link is based on the expressions in Chapter 2. It does not include the effects of residual intensity modulation, but includes the effect of the common-mode rejection ratio.

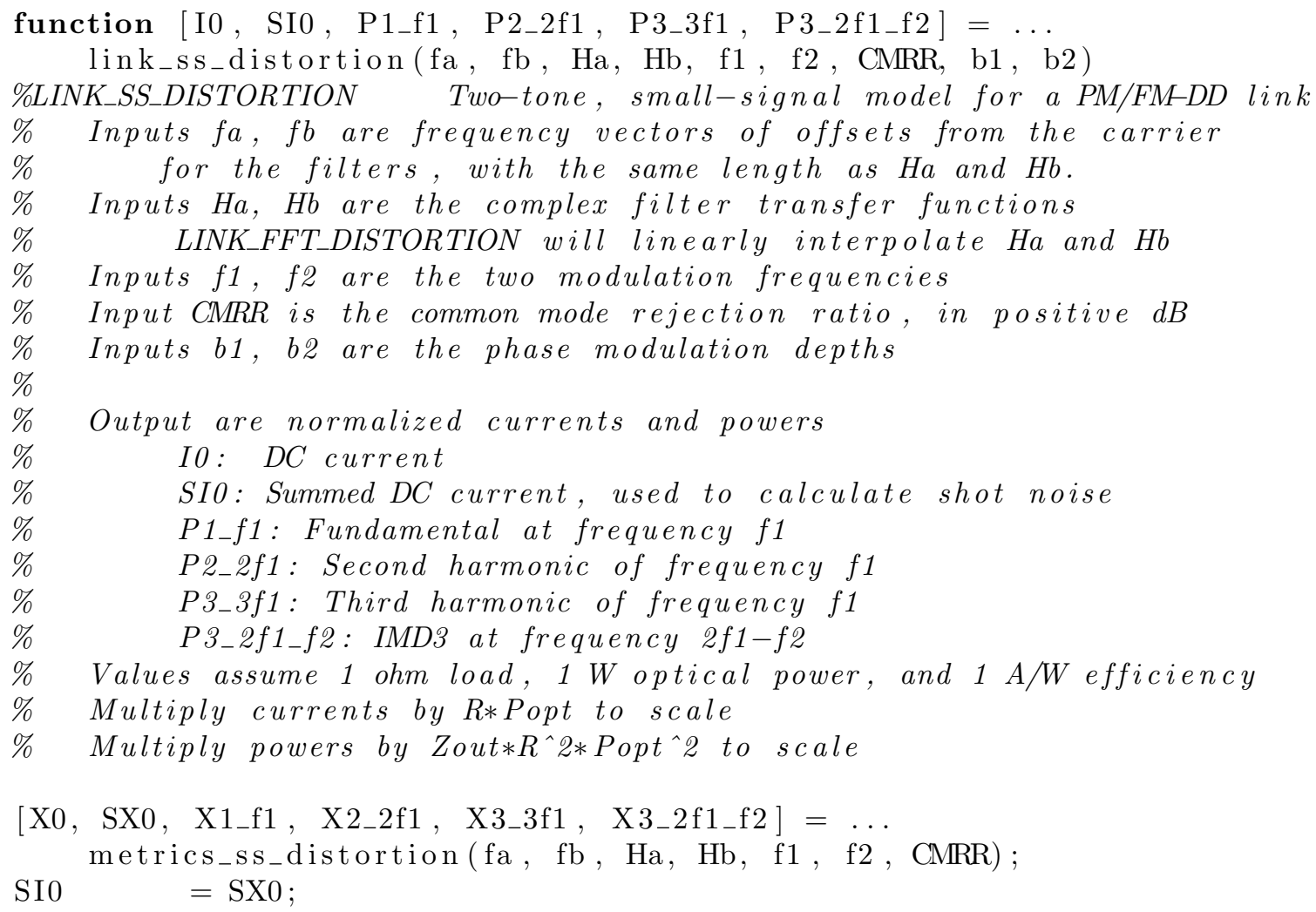




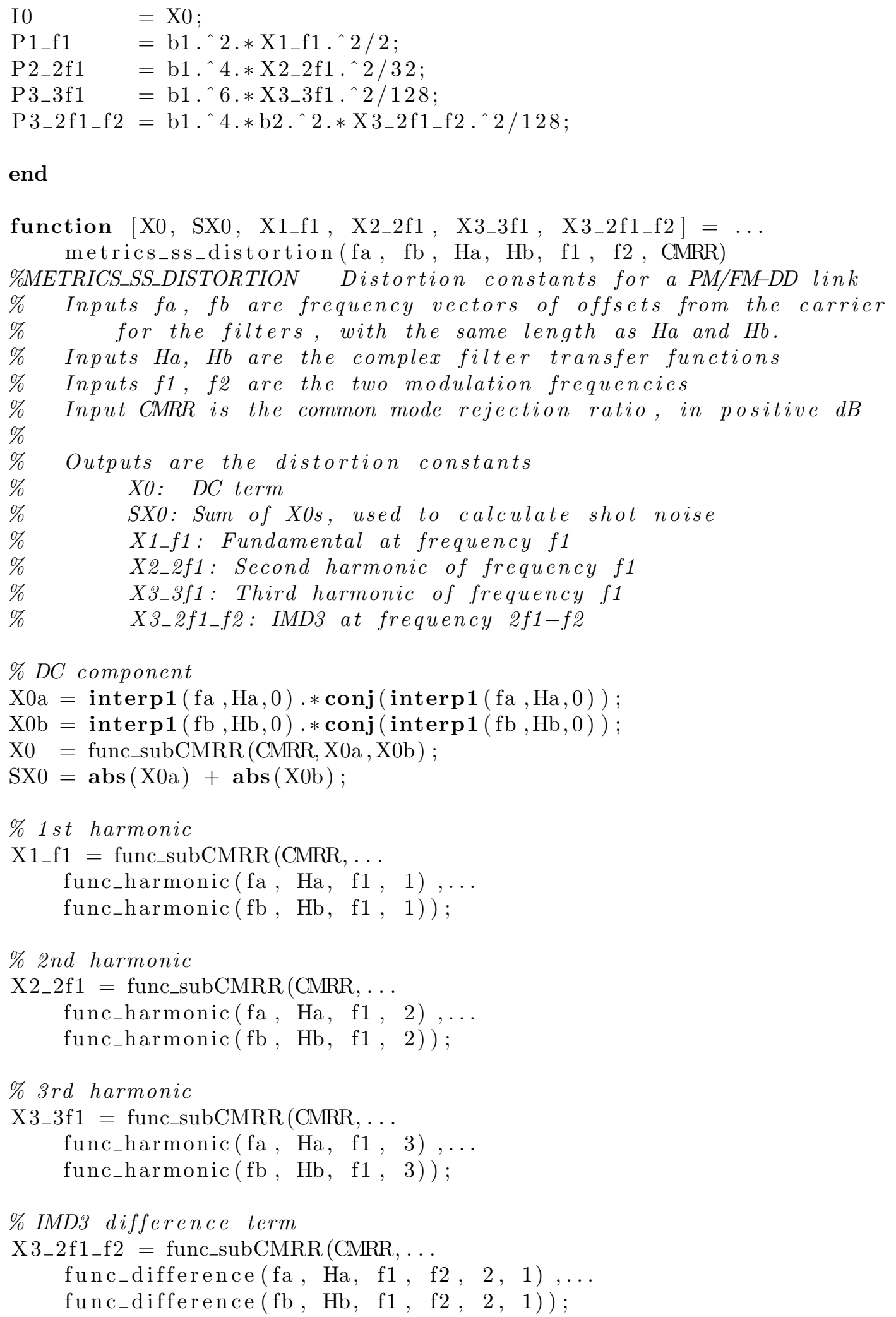

end

function $X=$ func_harmonic $(f, H, f 1, n)$ 


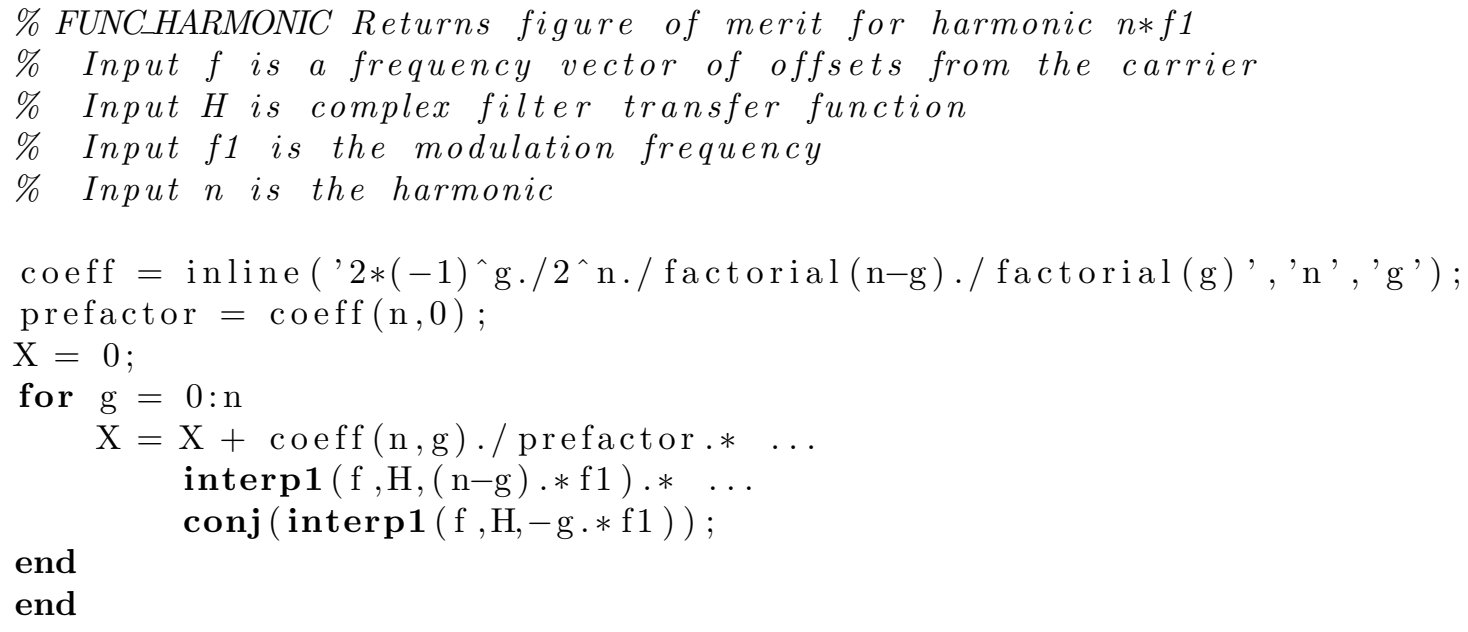

end

end

function $\mathrm{X}=$ func_difference $(\mathrm{f}, \mathrm{H}, \mathrm{f} 1, \mathrm{f} 2, \mathrm{n}, \mathrm{p})$

$\%$ FUNCDIFFERENCE Returns figure of merit for $n * f 1-p * f 2$

\% Input $f$ is a frequency vector of offsets from the carrier

$\%$ Input $H$ is complex filter transfer function

$\%$ Inputs $f 1$ and $f_{2}$ are the modulation frequencies

$\%$ Inputs $n$ and $p$ are the order

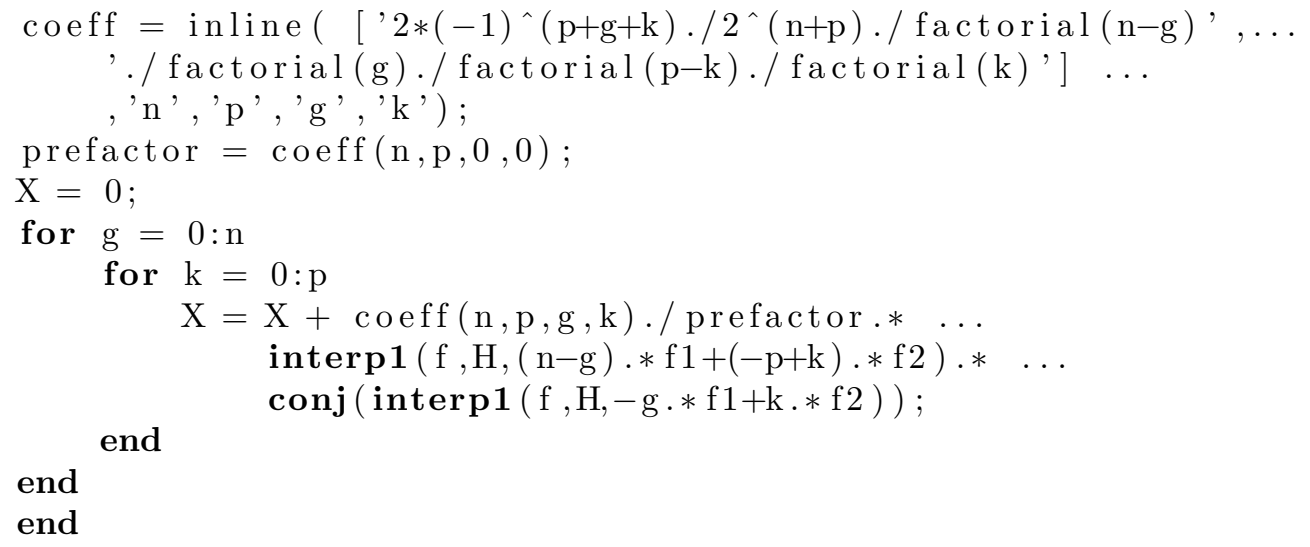




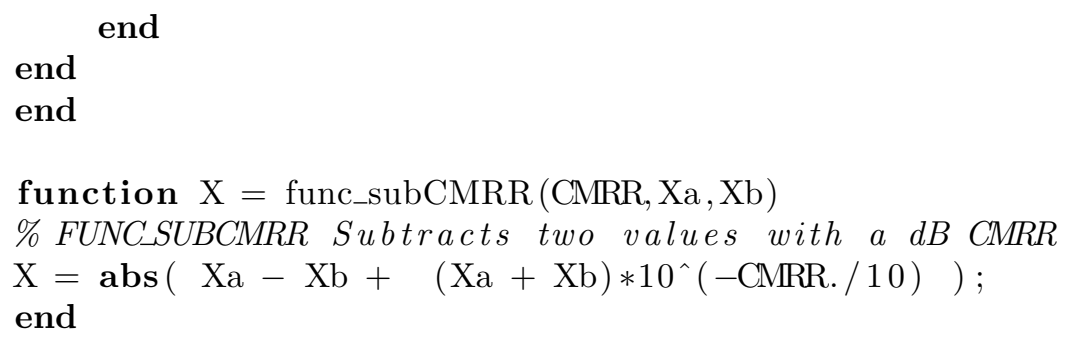

\section{A.2 Large-signal simulation}

The large signal model uses the expansion from (2.9).

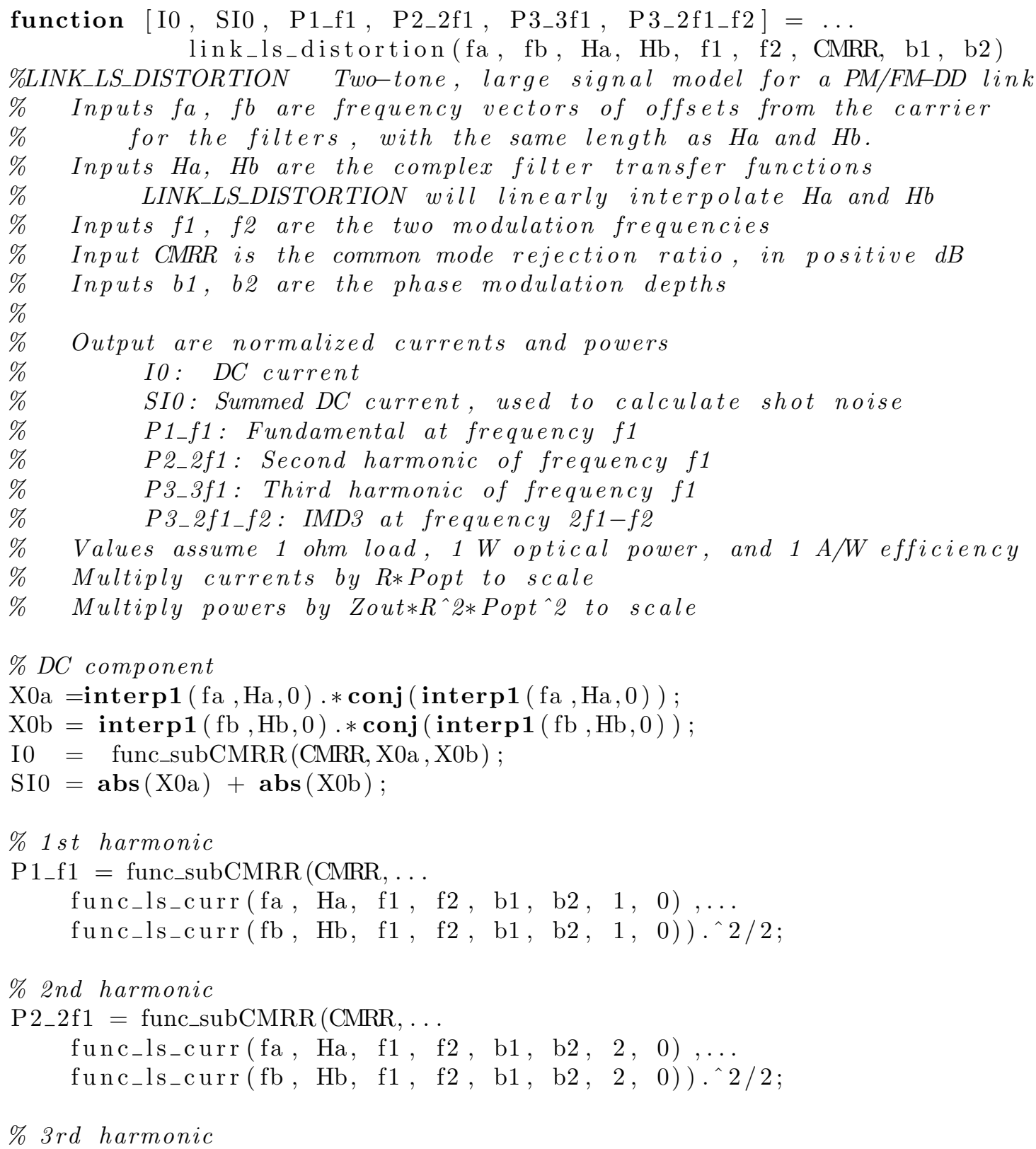




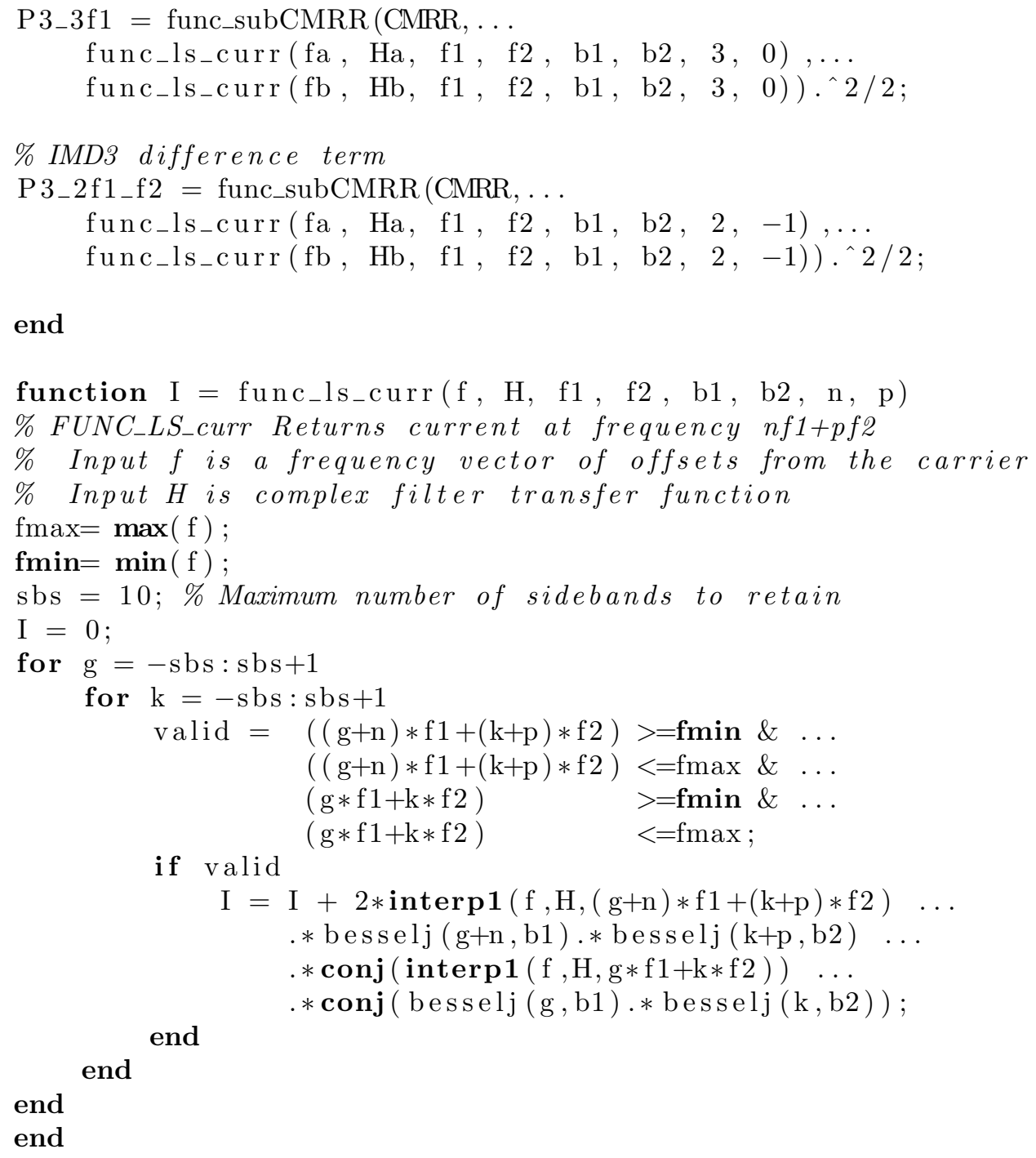

\section{A.3 Numerical simulation}

The numerical model for an FM link is based on the model in Figure 3.7 on page 49.

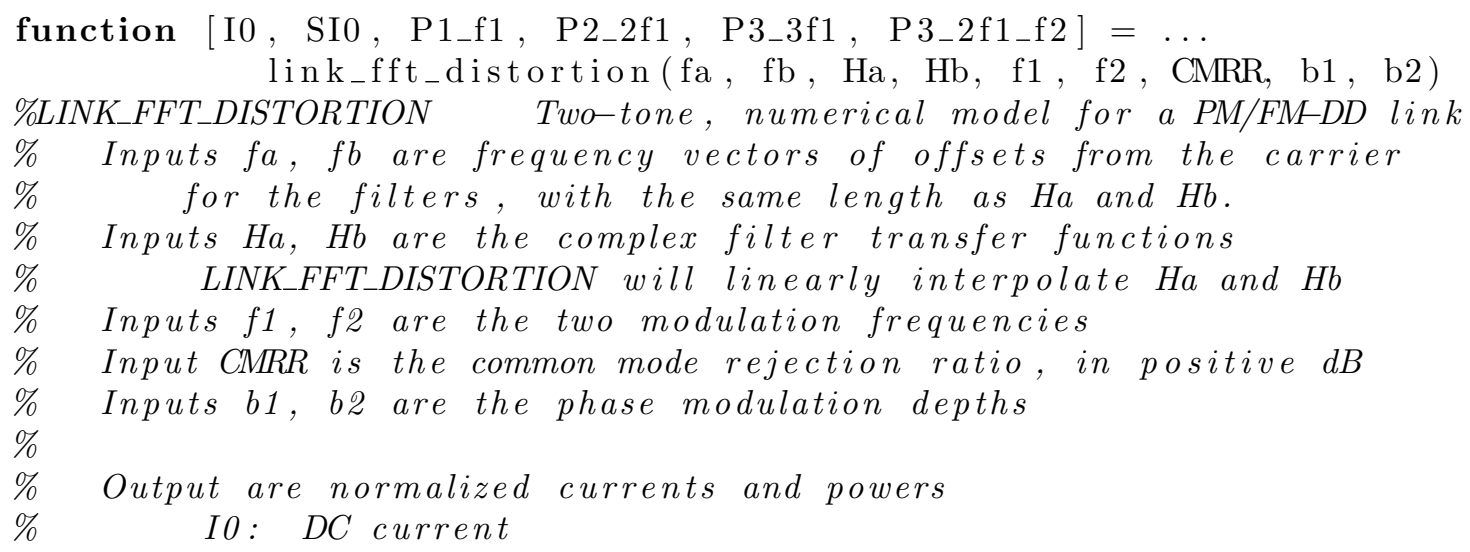




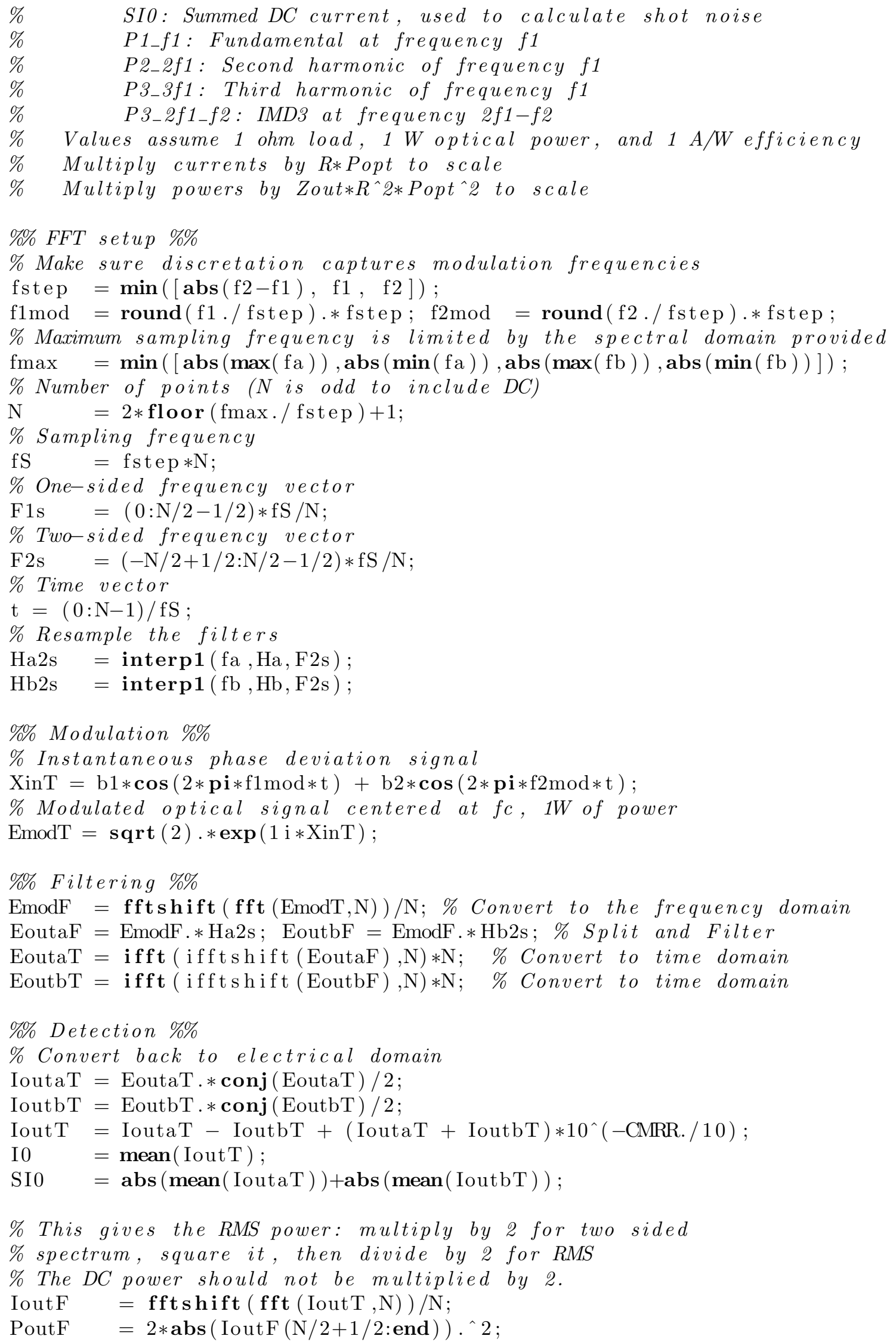




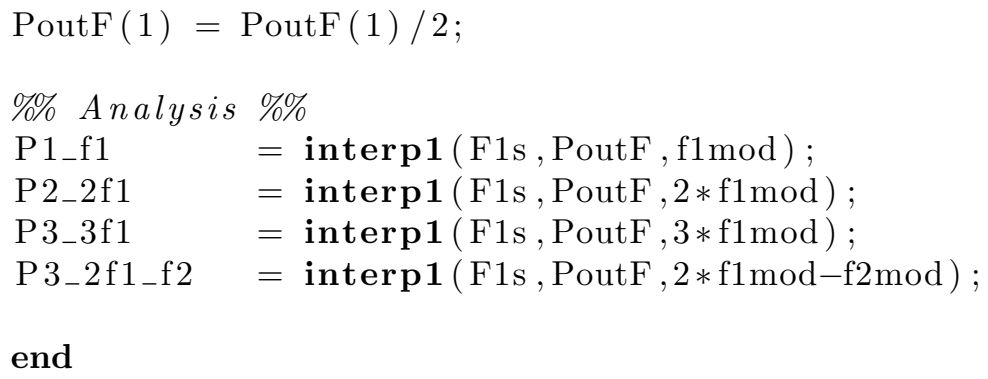

\section{A.4 Link response}

This program will output the response of the link, for a given input power, for any of the three models.

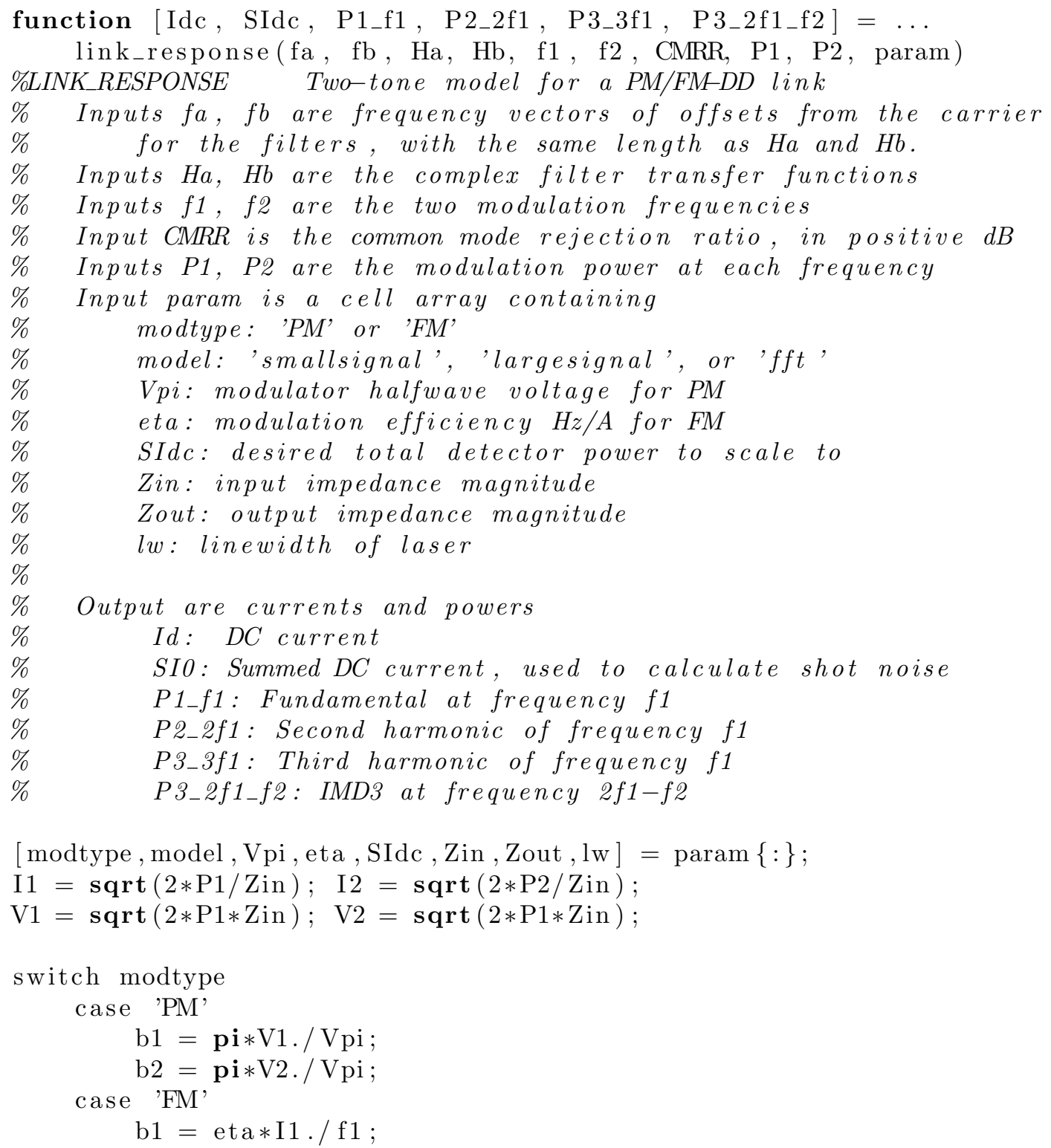


end $\quad \mathrm{b} 2=\mathrm{eta} * \mathrm{I} 2 . / \mathrm{f} 2 ;$

switch model

case 'smallsignal'

$\left[\mathrm{I} 0, \mathrm{SI} 0, \mathrm{P} 1 \_\mathrm{f} 1, \mathrm{P} 2 \_2 \mathrm{f} 1, \mathrm{P} 3 \_3 \mathrm{f} 1, \mathrm{P} 3 \_2 \mathrm{f} 1 \_\mathrm{f} 2\right]=\ldots$

link_ss_distortion( $\mathrm{fa}, \mathrm{fb}, \mathrm{Ha}, \mathrm{Hb}, \mathrm{f} 1, \mathrm{f} 2, \mathrm{CMRR}, \mathrm{b} 1, \mathrm{~b} 2)$;

case 'largesignal'

$\left[\mathrm{I} 0, \mathrm{SI} 0, \mathrm{P} 1 \_\mathrm{f} 1, \mathrm{P} 2 \_2 \mathrm{f} 1, \mathrm{P} 3 \_3 \mathrm{f} 1, \mathrm{P} 3 \_2 \mathrm{f} 1 \_\mathrm{f} 2\right]=\ldots$

link_ls_distortion(fa, fb, Ha, Hb, f1, f2, CMRR, b1, b2) ; case 'fft'

$\left[\mathrm{I} 0, \mathrm{SI} 0, \mathrm{P} 1 \_\mathrm{f} 1, \mathrm{P} 2 \_2 \mathrm{f} 1, \quad \mathrm{P} 3 \_3 \mathrm{f} 1, \quad \mathrm{P} 3 \_2 \mathrm{f} 1 \_\mathrm{f} 2\right]=\ldots$ link_fft_distortion(fa, fb, Ha, Hb, f1, f2, CMRR, b1, b2);

end

RPoptScale $=$ SIdc./SI0;

Idc $\quad=\mathrm{I} 0 *$ RPoptScale;

$\mathrm{P} 1 \_\mathrm{f} 1=\mathrm{P} 1 \_\mathrm{f} 1 . *$ Zout $*$ RPoptScale $\cdot{ }^{\wedge} 2$;

$\mathrm{P} 2 \_2 \mathrm{f} 1=\mathrm{P} 2 \_2 \mathrm{f} 1 * *$ Zout.$*$ RPoptScale ${ }^{\wedge} 2$;

P3_3f1 $=$ P 3_3f1 $*$ Zout.*RPoptScale .^ 2 ;

P3_2f1_f2 = P3_2f1_f2 * Zout.*RPoptScale .^2;

end

\section{A.5 Link metrics}

This program will calculate the small signal metrics of a given link.

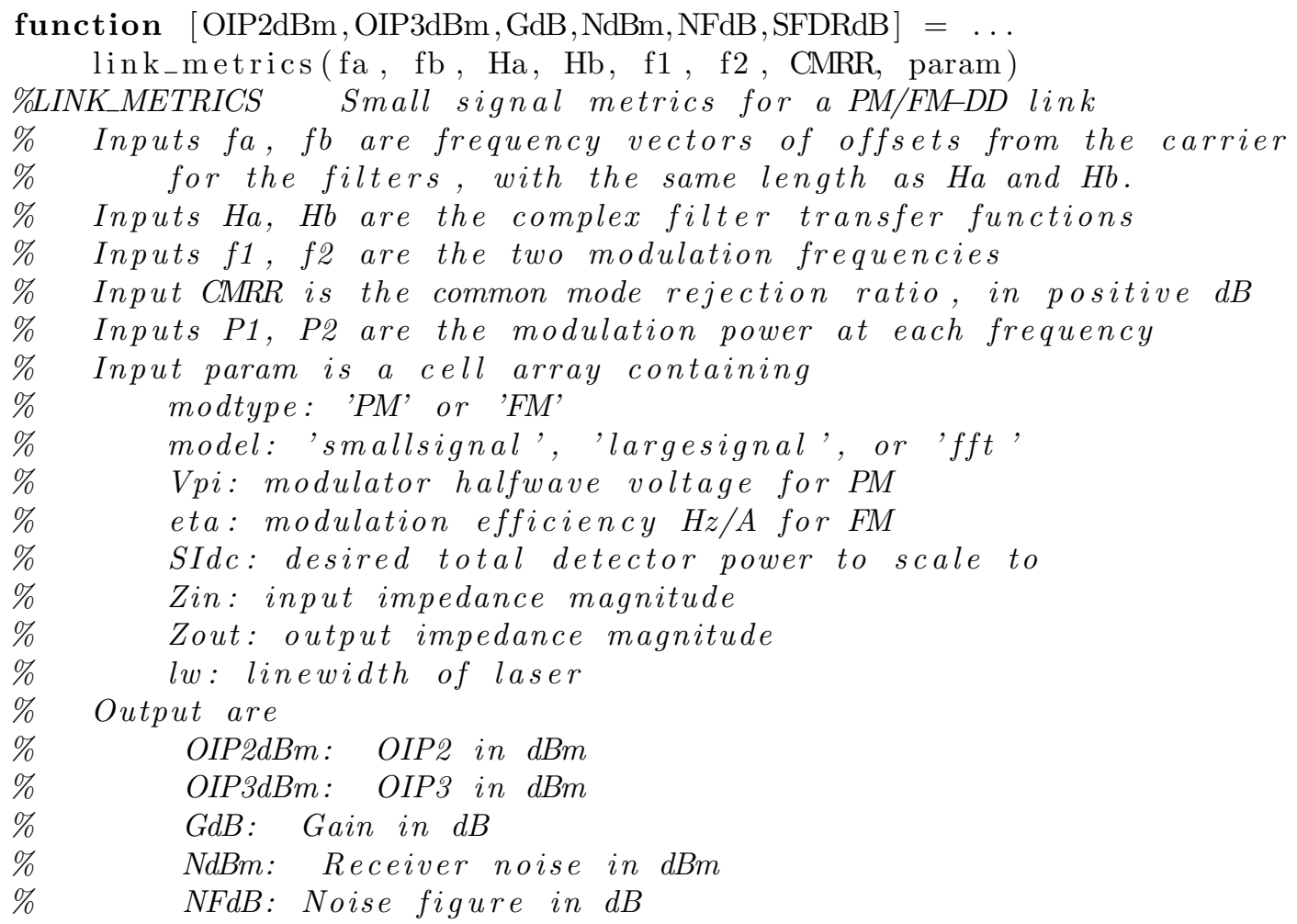




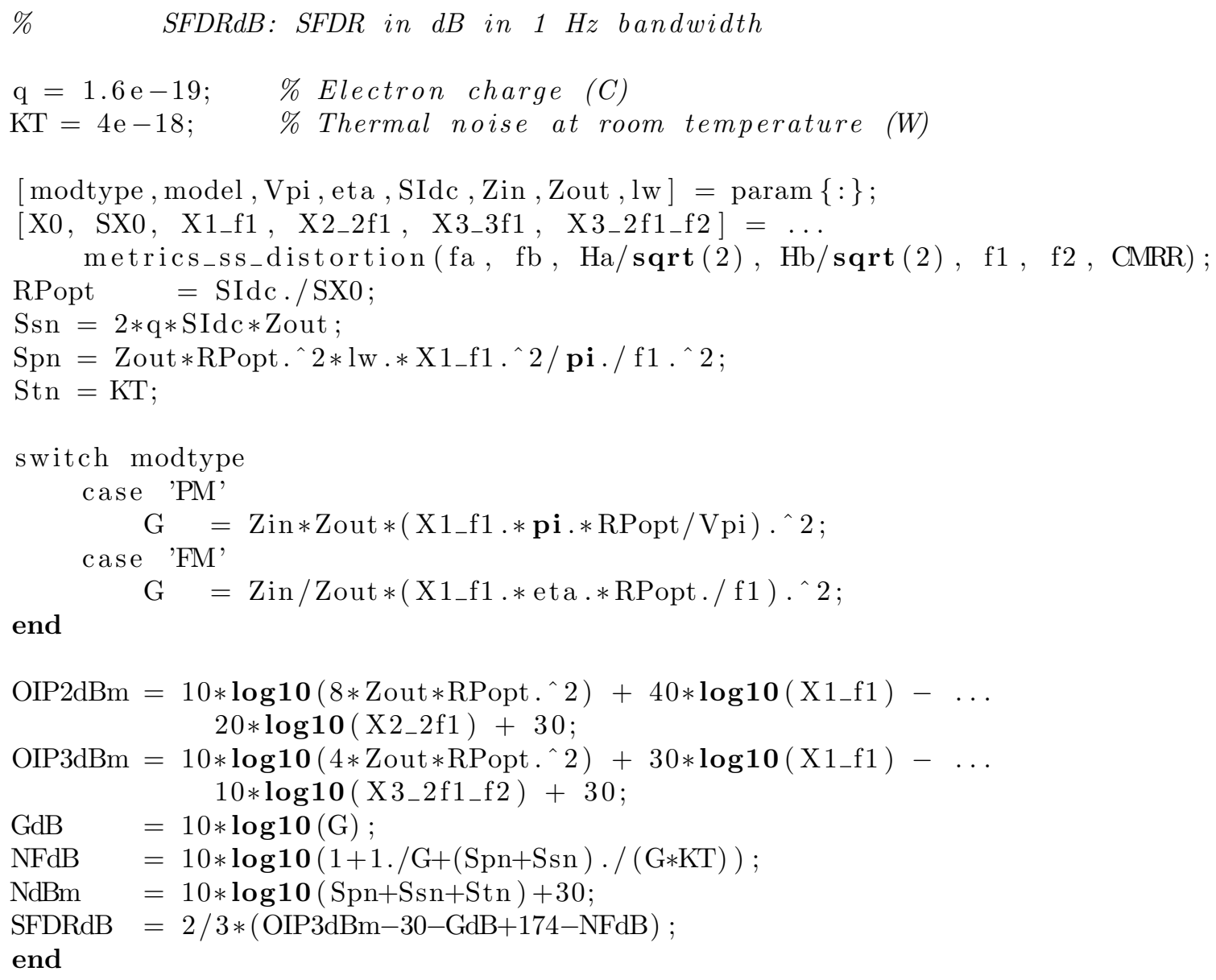




\section{Bibliography}

[1] A. J. Seeds and K. J. Williams, "Microwave Photonics," Lightwave Technology, Journal of, vol. 24, no. 12, pp. 4628 -4641, Dec 2006.

[2] J. Capmany and D. Novak, "Microwave photonics combines two worlds," Nature Photonics, vol. 1, no. 6, pp. 319-330, 2007. [Online]. Available: http://www.nature.com/doifinder/10.1038/nphoton.2007.89

[3] J. Yao, "Microwave Photonics," Lightwave Technology, Journal of, vol. 27, no. 3, pp. $314-335$, Feb 2009.

[4] C. Cox and E. Ackerman, "Microwave photonics: Past, present and future," in Microwave photonics, 2008. jointly held with the 2008 asia-pacific microwave photonics conference. mwp/apmp 2008. international topical meeting on, Oct 2008, pp. $9-11$.

[5] C. H. Cox, Analog Optical Links, Theory and Practice. Cambridge University Press, 2004.

[6] K. Y. Lau, Ultra-high Frequency Linear Fiber Optic Systems. Springer, 2009.

[7] M. Manka, "Microwave photonics for Electronic Warfare applications," in Microwave photonics, 2008. jointly held with the 2008 asia-pacific microwave photonics conference. mwp/apmp 2008. international topical meeting on, Oct 2008, pp. $275-278$.

[8] R. Minasian, "Photonic signal processing of microwave signals," Microwave Theory and Techniques, IEEE Transactions on, vol. 54, no. 2, pp. 832 - 846, Feb 2006.

[9] F. Zeng and J. Yao, "All-optical microwave mixing and bandpass filtering in a radio-over-fiber link," Photonics Technology Letters, IEEE, vol. 17, no. 4, pp. 899 -901, Apr 2005.

[10] B. Bortnik, I. Poberezhskiy, J. Chou, B. Jalali, and H. Fetterman, "Predistortion technique for RF-photonic generation of high-power ultrawideband arbitrary waveforms," Lightwave Technology, Journal of, vol. 24, no. 7, pp. 2752 - 2759, Jul 2006. 
[11] G. C. Valley, "Photonic analog-to-digital converters." Optics Express, vol. 15, no. 5, pp. 1955-1982, 2007. [Online]. Available: http://www.ncbi.nlm.nih.gov/ pubmed/19532436

[12] X. Yao and L. Maleki, "Optoelectronic oscillator for photonic systems," Quantum Electronics, IEEE Journal of, vol. 32, no. 7, pp. 1141 -1149, Jul 1996.

[13] J. Capmany and D. Novak, "Microwave photonics combines two worlds," Nature Photonics, vol. 1, no. 6, pp. 319-330, Jun 2007. [Online]. Available: http://www.nature.com/doifinder/10.1038/nphoton.2007.89

[14] P. Smyth, "Optical Radio - A Review of a Radical New Technology for Wireless Access Infrastructure," BT Technology Journal, vol. 21, pp. 22-31, 2003, 10.1023/A:1025198729319. [Online]. Available: http://dx.doi.org/10.1023/A: 1025198729319

[15] S. Pappert, C. Sun, R. Orazi, and T. Weiner, "Microwave fiber optic links for shipboard antenna applications," in IEEE International Conference on Phased Array Systems and Technology, 2000, 2000, pp. 345-348.

[16] I. Cox, C.H., E. Ackerman, G. Betts, and J. Prince, "Limits on the performance of RF-over-fiber links and their impact on device design," Microwave Theory and Techniques, IEEE Transactions on, vol. 54, no. 2, pp. 906 - 920, Feb 2006.

[17] V. Urick, F. Bucholtz, P. Devgan, J. McKinney, and K. Williams, "Phase Modulation With Interferometric Detection as an Alternative to Intensity Modulation With Direct Detection for Analog-Photonic Links," Microwave Theory and Techniques, IEEE Transactions on, vol. 55, no. 9, pp. 1978 -1985, Sep 2007.

[18] D. A. Tulchinsky, J. B. Boos, D. Park, P. G. Goetz, W. S. Rabinovich, and K. J. Williams, "High-Current Photodetectors as Efficient, Linear, and High-Power RF Output Stages," J. Lightwave Technol., vol. 26, no. 4, pp. 408-416, Feb 2008. [Online]. Available: http://jlt.osa.org/abstract.cfm?URI=jlt-26-4-408

[19] Z. Li, Y. Fu, M. Piels, H. Pan, A. Beling, J. E. Bowers, and J. C. Campbell, "High-power high-linearity flip-chip bonded modified uni-traveling carrier photodiode," Opt. Express, vol. 19, no. 26, pp. B385-B390, Dec 2011. [Online]. Available: http://www.opticsexpress.org/abstract.cfm?URI=oe-19-26-B385

[20] U. V. Cummings, "Linearized and high frequency electrooptic modulators," Ph.D. dissertation, California Institute of Technology, 2005. [Online]. Available: http://resolver.caltech.edu/CaltechETD:etd-05312005-225644

[21] J. Chen, R. Ram, and R. Helkey, "Linearity and third-order intermodulation distortion in DFB semiconductor lasers," Quantum Electronics, IEEE Journal of, vol. 35, no. 8, pp. 1231 -1237, Aug 1999. 
[22] X. J. Meng, T. Chau, and M. Wu, "Improved intrinsic dynamic distortions in directly modulated semiconductor lasers by optical injection locking," Microwave Theory and Techniques, IEEE Transactions on, vol. 47, no. 7, pp. $1172-1176$, Jul 1999.

[23] L. Roselli, V. Borgioni, F. Zepparelli, F. Ambrosi, M. Comez, P. Faccin, and A. Casini, "Analog laser predistortion for multiservice radio-over-fiber systems," Lightwave Technology, Journal of, vol. 21, no. 5, pp. 1211 - 1223, May 2003.

[24] V. Urick, M. Rogge, P. Knapp, L. Swingen, and F. Bucholtz, "Wide-band predistortion linearization for externally modulated long-haul analog fiber-optic links," Microwave Theory and Techniques, IEEE Transactions on, vol. 54, no. 4, pp. 1458 - 1463, Jun 2006.

[25] T. Ismail, C.-P. Liu, J. Mitchell, and A. Seeds, "High-Dynamic-Range WirelessOver-Fiber Link Using Feedforward Linearization," Lightwave Technology, Journal of, vol. 25, no. 11, pp. 3274 -3282, nov. 2007.

[26] A. Ramaswamy, L. Johansson, J. Klamkin, H.-F. Chou, C. Sheldon, M. Rodwell, L. Coldren, and J. Bowers, "Integrated Coherent Receivers for High-Linearity Microwave Photonic Links," Lightwave Technology, Journal of, vol. 26, no. 1, pp. $209-216$, Jan 2008.

[27] Y. Li, R. Wang, A. Bhardwaj, S. Ristic, and J. Bowers, "High Linearity InPBased Phase Modulators Using a Shallow Quantum-Well Design," Photonics Technology Letters, IEEE, vol. 22, no. 18, pp. 1340-1342, Sep 2010.

[28] T. Kakitsuka and S. Matsuo, "High-Speed Frequency Modulated DBR Lasers for Long-Reach Transmission," IEICE Transactions on Electronics, vol. E92-C, no. 7, pp. 929-936, Jul 2009.

[29] X. Xie, J. Khurgin, F.-S. Choa, X. Yu, J. Cai, J. Yan, X. Ji, Y. Gu, Y. Fang, Y. Sun, G. Ru, and Z. Chen, "A model for optimization of the performance of frequency-Modulated DFB semiconductor laser," IEEE Journal of Quantum Electronics, vol. 41, no. 4, pp. 473-482, 2005.

[30] Q. Xiang, Y. Zhao, and F.-S. Choa, "A high-performance RF-lightwave transmitter for analog fiber links," in Lasers and Electro-Optics Society 2000 Annual Meeting., vol. 1, 2000, pp. $138-139$.

[31] J. M. Roth, "Frequency modulated analog fiber optic links using direct detection," Master's thesis, MIT, Cambridge, May 1997.

[32] S. E. Harris, "Demodulation of phase-modulated light using birefringent crystals," Proceedings of the IEEE, vol. 52, no. 7, pp. 823 - 831, Jul 1964.

[33] S. E. Harris, E. O. Ammann, and I. C. Chang, "Optical Network Synthesis Using Birefringent Crystals. I. Synthesis of Lossless Networks of Equal-Length 
Crystals," J. Opt. Soc. Am., vol. 54, no. 10, pp. 1267-1278, 1964. [Online]. Available: http://www.opticsinfobase.org/abstract.cfm?URI=josa-54-10-1267

[34] I. P. Kaminow, "Balanced Optical Discriminator," Applied Optics, vol. 3, no. 4, p. 507, Apr 1964. [Online]. Available: http://www.opticsinfobase.org/abstract. cfm?URI=ao-3-4-507

[35] W. Way, Y. Lo, T. Lee, and C. Lin, "Direct detection of closely spaced optical FM-FDM Gb/s microwave PSK signals," IEEE Photonics Technology Letters, vol. 3, no. 2, pp. $176-178$, Feb 1991.

[36] P. Driessen, T. Darcie, and J. Zhang, "Analysis of a Class-B Microwave-Photonic Link Using Optical Frequency Modulation," IEEE/OSA Journal of Lightwave Technology, vol. 26, no. 15, pp. 2740 -2747, Aug 2008.

[37] D. Marpaung, C. Roeloffzen, A. Leinse, and M. Hoekman, "A photonic chip based frequency discriminator for a high performance microwave photonic link," Opt. Express, vol. 18, no. 26, pp. 27359-27370, Dec 2010. [Online]. Available: http://www.opticsexpress.org/abstract.cfm?URI=oe-18-26-27359

[38] X. Xie, J. Khurgin, J. Kang, and F.-S. Choa, "Ring-assisted frequency discriminator with improved linearity," IEEE Photonics Technology Letters, vol. 14, no. 8, pp. 1136-1138, Aug 2002. [Online]. Available: http: //ieeexplore.ieee.org/lpdocs/epic03/wrapper.htm?arnumber=1021994

[39] J. M. Wyrwas and M. C. Wu, "Dynamic Range of Frequency Modulated Direct-Detection Analog Fiber Optic Links," IEEE/OSA Journal of Lightwave Technology, vol. 27, no. 24, pp. 5552-5562, 2009. [Online]. Available: http://jlt.osa.org/abstract.cfm?URI=JLT-27-24-5552

[40] S. E. Harris, "Conversion of FM Light to AM Light Using Birefringent Crystals," Applied Physics Letters, vol. 2, no. 3, p. 47, 1963. [Online]. Available: http://link.aip.org/link/?APL/2/47/1\&Agg=doi

[41] S. Saito and T. Kimura, "Demodulation of phase-modulated optical maser beam by autocorrelation technique," Proceedings of the IEEE, vol. 52, no. 9, pp. 1048 - 1048, Sep 1964.

[42] S. Kobayashi, Y. Yamamoto, M. Ito, and T. Kimura, "Direct frequency modulation in AlGaAs semiconductor lasers," IEEE Journal of Quantum Electronics, vol. 18, no. 4, pp. 582 - 595, Apr 1982.

[43] S. Saito, Y. Yamamoto, and T. Kimura, "Semiconductor laser FSK modulation and optical direct discrimination detection," Electronics Letters, vol. 18, no. 11, pp. 468 -469, May 1982.

[44] W. Way, M. Maeda, A. Yi-Yan, M. Andrejco, M. Choy, M. Saifi, and C. Lin, "160-channel FM-video transmission using optical FM/FDM 
and subcarrier multiplexing and an erbium doped optical fibre amplifier," Electronics Letters, vol. 26, no. 2, pp. 139-142, 1990. [Online]. Available: http://link.aip.org/link/?ELL/26/139/1

[45] W. V. Sorin, K. W. Chang, G. A. Conrad, and P. R. Hernday, "Frequency domain analysis of an optical FM discriminator," IEEE/OSA Journal of Lightwave Technology, vol. 10, no. 6, pp. 787-793, Jun 1992.

[46] G. Fiksman, R. Gross, J. Fan, and L. Kazovsky, "Performance optimization of directly modulated FM-SCM systems with optical discriminator," IEEE Photonics Technology Letters, vol. 5, no. 7, pp. 845 -848, Jul 1993.

[47] S. Woodward, "Lightwave CATV systems using frequency-modulated laser and interferometer," Electronics Letters, vol. 25, no. 24, pp. 1665 -1666, Nov 1989.

[48] G. Yabre and J. Le Bihan, "Intensity modulation technique using a directly frequency-modulated semiconductor laser and an interferometer," Lightwave Technology, Journal of, vol. 13, no. 10, pp. 2093 -2098, Oct 1995.

[49] K. Tsukamoto, S. Fujii, P. Sanjo, and S. Komaki, "Theoretical Consideration On Nonlinear Distortion Suppression In Directly Optical FM Microwave over Fiber System," in International Topical Meeting on Microwave Photonics, Sep 1997, pp. $251-255$.

[50] J. Chen and R. Brown, "Novel optical frequency discriminator with "perfect" linearity," in Optical Fiber Communication Conference, vol. 2, 2000, pp. 329 -331 .

[51] X. Xie, J. Khurgin, J. Kang, and F. Choa, "Compact linearized optical FM discriminator," IEEE Photonic. Tech. L., vol. 14, no. 3, pp. 384-386, Mar 2002. [Online]. Available: http://ieeexplore.ieee.org/lpdocs/epic03/wrapper. htm?arnumber $=986821$

[52] G. Chen, J. Kang, and J. Khurgin, "Frequency discriminator based on ring-assisted fiber sagnac filter," IEEE Photonics Technology Letters, vol. 17, no. 1, pp. 109-111, Jan 2005. [Online]. Available: http: / /ieeexplore.ieee.org/lpdocs/epic03/wrapper.htm?arnumber=1372599

[53] D. Marpaung, C. Roeloffzen, R. Timens, A. Leinse, and M. Hoekman, "Design and realization of an integrated optical frequency modulation discriminator for a high performance microwave photonic link," in Microwave Photonics (MWP), 2010 IEEE Topical Meeting on, Oct 2010, pp. 131 -134.

[54] T. Darcie, J. Zhang, P. Driessen, and J. Eun, "Demonstration of a class-B microwave-photonic link using optical frequency modulation and complementary fiber-Bragg-grating discriminators," in Optical Fiber Communication Conference, Mar 2006, pp. 1 - 3. 
[55] J. Zhang and T. Darcie, "Low-biased microwave-photonic link using optical frequency or phase modulation and fiber-Bragg-grating discriminator," in Optical Fiber Communication and the National Fiber Optic Engineers Conference, Mar 2006, p. 3 pp.

[56] — - "Clipping-Free Dynamic Range: the Fundamental Limit for Class-B Microwave-Photonic Links," in International Topical Meeting on Microwave Photonics, Oct 2006, pp. $1-4$.

[57] T. Darcie, J. Zhang, P. Driessen, and J.-J. Eun, "Class-B Microwave-Photonic Link Using Optical Frequency Modulation and Linear Frequency Discriminators," IEEE/OSA Journal of Lightwave Technology, vol. 25, no. 1, pp. 157-164, Jan 2007.

[58] J. Zhang, A. Hone, and T. Darcie, "Limitation Due to Signal-Clipping in Linearized Microwave-Photonic Links," IEEE Photonics Technology Letters, vol. 19, no. 14, pp. 1033 -1035, Jul 2007.

[59] J. Zhang and T. Darcie, "Two-Tone Analysis of Distortion Suppression in Microwave-Photonic Links Using Phase Modulation and Fiber-Bragg Grating Filters," in International Symposium on Signals, Systems and Electronics, Jul 2007, pp. $621-624$.

[60] J. Zhang, T. Darcie, and J. J. Eun, "High-Performance Passive MicrowavePhotonic Link for Antenna Remoting Using Truncated Single-Sideband Optical Phase Detection," in Optical Fiber Communication and the National Fiber Optic Engineers Conference, Mar 2007, pp. 1-3.

[61] I. Gasulla and J. Capmany, "Analytical model and figures of merit for filtered Microwave photonic links," Opt. Express, vol. 19, no. 20, pp. 19758-19774, Sep 2011. [Online]. Available: http://www.opticsexpress.org/abstract.cfm?URI= oe-19-20-19758

[62] R. F. Kalman, J. C. Fan, and L. G. Kazovsky, "Dynamic range of coherent analog fiber-optic links," IEEE/OSA Journal of Lightwave Technology, vol. 12, no. 7, pp. 1263-1277, 1994.

[63] J. Capmany, B. Ortega, and D. Pastor, "A Tutorial on Microwave Photonic Filters," J. Lightwave Technol., vol. 24, no. 1, p. 201, Jan 2006. [Online]. Available: http://jlt.osa.org/abstract.cfm?URI=jlt-24-1-201

[64] C. K. Madsen and J. H. Zhao, Optical Filter Design and Analysis. John Wiley \& Sons, Inc., 1999.

[65] J. M. Wyrwas and M. C. Wu, "High Dynamic-Range Microwave Photonic Links Using Maximally Linear FIR Optical Filters," in Optical Fiber Communication Conference. Optical Society of America, 2010, p. JWA43. [Online]. Available: http://www.opticsinfobase.org/abstract.cfm?URI=OFC-2010-JWA43 
[66] B. Kumar and S. Dutta Roy, "Design of digital differentiators for low frequencies," Proceedings of the IEEE, vol. 76, no. 3, pp. 287 -289, Mar 1988.

[67] B. Kumar and S. C. Dutta Roy, "Maximally linear FIR digital differentiators for midband frequencies," International Journal of Circuit Theory and Applications, vol. 17, no. 1, pp. 21-27, Nov 1989. [Online]. Available: http://dx.doi.org/10.1002/cta.4490170104

[68] B. Kumar, S. Dutta Roy, and H. Shah, "On the design of FIR digital differentiators which are maximally linear at the frequency pi/p," Signal Processing, IEEE Transactions on, vol. 40, no. 9, pp. 2334 -2338, Sep 1992.

[69] K. Takiguchi, K. Jinguji, K. Okamoto, and Y. Ohmori, "Variable group-delay dispersion equalizer using lattice-form programmable optical filter on planar lightwave circuit," IEEE Journal of Selected Topics in Quantum Electronics, vol. 2, no. 2, pp. 270 -276, Jun 1996.

[70] D. Gill, S. Patel, M. Rasras, K.-Y. Tu, A. White, Y.-K. Chen, A. Pomerene, D. Carothers, R. Kamocsai, C. Hill, and J. Beattie, "CMOS-Compatible SiRing-Assisted Mach-Zehnder Interferometer With Internal Bandwidth Equalization," Selected Topics in Quantum Electronics, IEEE Journal of, vol. 16, no. 1, pp. $45-52$, Jan 2010.

[71] C. R. Doerr and K. Okamoto, "Advances in Silica Planar Lightwave Circuits," Lightwave Technology, Journal of, vol. 24, no. 12, pp. 4763 -4789, Dec 2006.

[72] K. Takiguchi, S. Kawanishi, H. Takara, A. Himeno, and K. Hattori, "Dispersion slope equalizer for dispersion shifted fiber using a lattice-form programmable optical filter on a planar lightwave circuit," IEEE/OSA Journal of Lightwave Technology, vol. 16, no. 9, pp. 1647 -1656, Sep 1998.

[73] K. Takiguchi, K. Okamoto, and T. Goh, "Planar lightwave circuit dispersion equaliser with reduced bias electrical power employing phase trimming technique," Electronics Letters, vol. 36, no. 7, pp. 657-658, 2000. [Online]. Available: http://link.aip.org/link/?ELL/36/657/1

[74] — - "Integrated optic dispersion slope equaliser for N x 20 Gbit/s WDM transmission," Electronics Letters, vol. 37, no. 11, pp. 701-703, 2001. [Online]. Available: http://link.aip.org/link/?ELL/37/701/1

[75] K. Takiguchi, K. Okamoto, T. Goh, and M. Itoh, "Integrated-Optic Dispersion Slope Equalizer for N x Several Tens of Gb/s WDM Transmission," IEEE/OSA Journal of Lightwave Technology, vol. 21, no. 11, p. 2463, 2003. [Online]. Available: http://jlt.osa.org/abstract.cfm?URI=JLT-21-11-2463

[76] K. Takiguchi, H. Takahashi, and T. Shibata, "Tunable chromatic dispersion and dispersion slope compensator using a planar lightwave circuit lattice-form filter," Optics Letters, vol. 33, no. 11, pp. 1243-1245, 2008. [Online]. Available: http://ol.osa.org/abstract.cfm?URI=ol-33-11-1243 
[77] G.-L. Bona, R. Germann, and B. J. Offrein, "SiON high-refractive-index waveguide and planar lightwave circuits," IBM Journal of Research and Development, vol. 47, no. 2.3, pp. 239 -249, Mar 2003.

[78] M. Bohn and C. Xia, "Electrical and optical equalization strategies in direct detected high-speed transmission systems," AEU - International Journal of Electronics and Communications, vol. 63, pp. 526 - 532, 2009. [Online]. Available: http://www.sciencedirect.com/science/article/ B7GWW-4WD115T-1/2/1399a6fa79891bafae321ff2c9d0d92d

[79] B. Offrein, F. Horst, G. Bona, R. Germann, H. Salemink, and R. Beyeler, "Adaptive gain equalizer in high-index-contrast SiON technology," IEEE Photonics Technology Letters, vol. 12, no. 5, pp. 504 -506, May 2000.

[80] M. Bohn, W. Rosenkranz, and P. Krummrich, "Adaptive distortion compensation with integrated optical finite impulse response filters in high bitrate optical communication systems," IEEE Journal of Selected Topics in Quantum Electronics, vol. 10, no. 2, pp. 273 - 280, Mar 2004.

[81] M. Bohn and W. Rosenkranz, "Experimental verification of combined adaptive PMD and GVD compensation in a $40 \mathrm{~Gb} / \mathrm{s}$ transmission using integrated optical FIR-filters and spectrum monitoring," in Optical Fiber Communication Conference, vol. 1, Feb 2004, p. 2 vol. (1800).

[82] M. Bohn, P. Krurnmrich, and W. Rosenkranz, "Automatic control of optical equalizers," in Optical Fiber Communication Conference, vol. 3, Mar 2005, p. 3 pp. Vol. 3.

[83] M. Bohn, G. Mohs, C. Scheerer, C. Glingener, C. Wree, and W. Rosenkranz, "An adaptive optical equalizer concept for single channel distortion compensation," in 27th European Conference on Optical Communication, vol. 1, 2001, pp. 6 7 vol.1.

[84] M. Bohn, W. Rosenkranz, and G. Mohs, "Multispan inline and adaptive group delay ripple equalization concepts @ 40 Gb/s with optical FIR-filters," in Optical Fiber Communication Conference, mar. 2002, pp. 665 - 667.

[85] F. Horst, R. Germann, U. Bapst, D. Wiesmann, B. Offrein, and G. Bona, "Compact tunable FIR dispersion compensator in SiON technology," IEEE Photonics Technology Letters, vol. 15, no. 11, pp. 1570 -1572, Nov 2003.

[86] M. Secondini, E. Forestieri, and G. Prati, "Performance of MSE configured PLC optical equalizers for chromatic dispersion compensation," IEEE Photonics Technology Letters, vol. 15, no. 2, pp. 248 -250, Feb 2003.

[87] — _ "Adaptive minimum MSE controlled PLC optical equalizer for chromatic dispersion compensation," IEEE/OSA Journal of Lightwave Technology, vol. 21, no. 10 , pp. $2322-2331$, Oct 2003 . 
[88] — - "PLC optical equalizer for chromatic and polarization-mode dispersion compensation based on MSE control," IEEE Photonics Technology Letters, vol. 16, no. 4, pp. $1173-1175$, Apr 2004.

[89] K. Takiguchi, S. Suzuki, and T. Shibata, "Method for adjusting lattice-form optical devices and its use in realising low-loss variable chromatic dispersion compensator," Electronics Letters, vol. 39, no. 4, pp. 355 - 356, Feb 2003.

[90] V. Houtsma, T. Hu, N. Weimann, R. Kopf, A. Tate, J. Frackoviak, R. Reyes, Y. Chen, and L. Zhang, "A $1 \mathrm{~W}$ linear high-power InP balanced uni-traveling carrier photodetector," in Optical Communication (ECOC), 2011 37th European Conference and Exhibition on, Sep 2011, pp. 1-3.

[91] L. A. Johansson, J. S. Barton, L. A. Coldren, and G. A. Fish, "High-speed optical frequency modulation in a monolithically integrated widely-tunable laserphase modulator," in Proc. Optical Fiber Communication Conf. OFC 2004, vol. 2, 2004.

[92] M. N. Sysak, L. A. Johansson, L. A. Coldren, M. Rodwell, and J. E. Bowers, "A High Efficiency, Current Injection Based Quantum-Well Phase Modulator Monolithically Integrated with a Tunable Laser for Coherent Systems," in Optical Amplifiers and Their Applications/Coherent Optical Technologies and Applications. Optical Society of America, 2006, p. CFC6. [Online]. Available: http://www.opticsinfobase.org/abstract.cfm?URI=COTA-2006-CFC6

[93] M. N. Sysak, L. Johansson, J. S. Klamkin, L. A. Coldren, and J. E. Bowers, "A Dynamic Measurement Technique for Third-Order Distortion in Optical Phase Modulators," IEEE Photonics Technology Letters, vol. 19, no. 3, pp. 170-172, 2007.

[94] J. Le Bihan, G. Yabre, J. Debeau, and E. Le Coquil, "Bessel function analysis of harmonic distortion in semiconductor lasers," Electronics Letters, vol. 29, no. 10, pp. 834-835, 1993.

[95] J. Le Bihan and G. Yabre, "FM and IM intermodulation distortions in directly modulated single-mode semiconductor lasers," IEEE Journal of Quantum Electronics, vol. 30, no. 4, pp. 899-904, 1994.

[96] — - "Distortion in a semiconductor laser under quasi-sinusoidal current modulation," in Proc. SBMO/IEEE MTT-S Int Microwave and Optoelectronics Conf., vol. 2, 1995, pp. 932-935.

[97] G. Yabre, "Nonlinear distortion of a three-tone modulated laser diode followed by an interferometer," Applied Physics Letters, vol. 69, no. 12, pp. 1671-1673, 1996.

[98] — - "Improved direct-modulation characteristics of a semiconductor laser by FM/IM conversion through an interferometer," IEEE/OSA Journal of Lightwave Technology, vol. 14, no. 10, pp. 2135-2140, 1996. 
[99] — - "Interferometric conversion of laser chirp to IM: effect of the interferometer free spectral range on the output nonlinear distortion," IEEE Photonics Technology Letters, vol. 8, no. 10, pp. 1388-1390, 1996.

[100] — "Third-order Intermodulation Distortion And Noise Behavior Of Laser Diode Transmitters Using Optical FM demodulation," in Proc. Int Microwave Photonics MWP '977 Topical Meeting, 1997, pp. 247-250.

[101] — "Analog direct-modulation behavior of semiconductor laser transmitters using optical FM demodulation," IEEE/OSA Journal of Lightwave Technology, vol. 16, no. 3, pp. 411-418, 1998.

[102] — - "Output intensity noise of lightwave transmitters employing chirp demodulation," IEEE Photonics Technology Letters, vol. 10, no. 7, pp. 1024-1026, 1998.

[103] T. Wolf, K. Drogemuller, B. Borchert, H. Westermeier, E. Veuhoff, and H. Baumeister, "Tunable twin-guide lasers with flat frequency modulation response by quantum confined Stark effect," Applied Physics Letters, vol. 60, no. 20, pp. 2472-2474, 1992.

[104] X. Huang, A. J. Seeds, and J. S. Roberts, "Reverse bias tuned multiple quantum well ridge guide laser with uniform frequency modulation response," Applied Physics Letters, vol. 71, no. 6, pp. 765-766, 1997.

[105] X. Huang, A. J. Seeds, J. S. Roberts, and A. P. Knights, "Monolithically integrated quantum-confined stark effect tuned laser with uniform frequency modulation response," IEEE Photonics Technology Letters, vol. 10, no. 12, pp. 16971699, 1998.

[106] S. Murata, I. Mito, and K. Kobayashi, "Spectral characteristics for $1.5 \mu \mathrm{m}$ DBR laser with frequency-tuning region," IEEE Journal of Quantum Electronics, vol. 23, no. 6, pp. 835-838, 1987.

[107] O. Ishida, H. Toba, and Y. Tohmori, "Pure frequency modulation of a multielectrode distributed-Bragg-reflector (DBR) laser diode," IEEE Photonics Technology Letters, vol. 1, no. 7, pp. 156-158, 1989.

[108] O. Ishida, H. Toba, Y. Tohmori, and K. Oe, "Multielectrode DBR laser diode for wide bandwidth and flat FM response," Electronics Letters, vol. 25, no. 11, pp. 703-704, 1989.

[109] A. Zatni and J. Le Bihan, "Analysis of FM and AM responses of a tunable threeelectrode DBR laser diode," IEEE Journal of Quantum Electronics, vol. 31, no. 6, pp. 1009-1014, 1995.

[110] J. Langanay, E. Gaumont-Goarin, J. Y. Emery, C. Labourie, J. G. Provost, C. Starck, O. Le Gouezigou, and D. Lesterlin, "High FM bandwidth of DBR 
laser including butt-jointed electro-optical wavelength tuning sections," Electronics Letters, vol. 30, no. 4, pp. 311-312, 1994.

[111] F. Delorme, S. Slempkes, A. Ramdane, B. Rose, and K. Nakajima, "Subnanosecond tunable distributed Bragg reflector lasers with an electrooptical Bragg section," IEEE Journal of Selected Topics in Quantum Electronics, vol. 1, no. 2, pp. 396-400, 1995.

[112] F. Delorme, A. Ramdane, B. Rose, S. Slempkes, and H. Nakajima, "Ultrafast optical switching operation of DBR lasers using an electro-optical tuning section," IEEE Photonics Technology Letters, vol. 7, no. 3, pp. 269-271, 1995.

[113] G. Alibert, F. Delorme, P. Boulet, J. Landreau, and H. Nakajima, "Subnanosecond tunable laser using a single electroabsorption tuning super structure grating," IEEE Photonics Technology Letters, vol. 9, no. 7, pp. 895-897, 1997.

[114] G. Alibert, F. Delorme, S. Grosmaire, S. Slempkes, A. Ougazzaden, and H. Nakajima, "A new tunable laser using a single electroabsorption tuning super structure grating for subnanosecond switching applications," IEEE Journal of Selected Topics in Quantum Electronics, vol. 3, no. 2, pp. 598-606, 1997.

[115] X. Xie, J. B. Khurgin, and F.-S. Choa, "Suppression of spurious intensity modulation in frequency-modulated semiconductor lasers," IEEE Journal of Selected Topics in Quantum Electronics, vol. 9, no. 5, pp. 1294-1299, 2003.

[116] M. Pantouvaki, C. C. Renaud, P. Cannard, M. J. Robertson, R. Gwilliam, and A. J. Seeds, "Fast Tuneable InGaAsP DBR Laser Using Quantum-Confined Stark-Effect-Induced Refractive Index Change," IEEE Journal of Selected Topics in Quantum Electronics, vol. 13, no. 5, pp. 1112-1121, 2007.

[117] S. Matsuo, T. Kakitsuka, T. Segawa, N. Fujiwara, Y. Shibata, H. Yasaka, and H. Suzuki, "Frequency Modulated Widely Tunable SSG-DBR Laser with Optical Filtering for Extended Transmission Reach," in Proc. 20th Annual Meeting of the IEEE Lasers and Electro-Optics Society LEOS 2007, 2007, pp. 494-495.

[118] S. Matsuo, T. Kakitsuka, T. Segawa, N. Fujiwara, Y. Shibata, H. Oohashi, H. Yasaka, and H. Suzuki, "Extended Transmission Reach Using Optical Filtering of Frequency-Modulated Widely Tunable SSG-DBR Laser," IEEE Photonics Technology Letters, vol. 20, no. 4, pp. 294-296, 2008.

[119] S. Matsuo, T. Kakitsuka, T. Segawa, R. Sato, Y. Shibata, R. Takahashi, H. Oohashi, and H. Yasaka, "4 x $25 \mathrm{~Gb} / \mathrm{s}$ Frequency-Modulated DBR Laser Array for 100-GbE 40-km Reach Application," IEEE Photonics Technology Letters, vol. 20, no. 17, pp. 1494-1496, 2008.

[120] W. V. Sorin, K. W. Chang, G. A. Conrad, and P. R. Hernday, "Frequency domain analysis of an optical FM discriminator," IEEE/OSA Journal of Lightwave Technology, vol. 10, no. 6, pp. 787-793, 1992. 
[121] S. Murata, I. Mito, and K. Kobayashi, "Frequency modulation and spectral characteristics for a $1.5 \mu \mathrm{m}$ phase-tunable DFB laser," Electronics Letters, vol. 23, no. 1, pp. 12-14, 1987.

[122] M. Okai, M. Suzuki, and M. Aoki, "Complex-coupled $\lambda / 4$-shifted DFB lasers with a flat FM response," IEEE Journal of Selected Topics in Quantum Electronics, vol. 1, no. 2, pp. 461-465, 1995.

[123] J. B. Khurgin, X. Xie, F. Choa, X. Yu, J. Cai, J. Yan, X. Ji, Y. Gu, Y. Fang, Y. Sun, and Z. Chen, "Optimization of the performance of frequency-modulated DFB semiconductor laser: theory and experiment," in Proc. (CLEO) Lasers and Electro-Optics Conf, vol. 2, 2005, pp. 1539-1541.

[124] S. Ogita, Y. Kotaki, M. Matsuda, Y. Kuwahara, H. Onaka, H. Miyata, and H. Ishikawa, "FM response of narrow-linewidth, multielectrode $\lambda / 4$ shift DFB laser," IEEE Photonics Technology Letters, vol. 2, no. 3, pp. 165-166, 1990.

[125] M. Horita, M. Tsurusawa, K. Utaka, and Y. Matsushima, "Wavelength-tunable InGaAsP-InP multiple- $\lambda / 4$-shifted distributed feedback laser," IEEE Journal of Quantum Electronics, vol. 29, no. 6, pp. 1810-1816, 1993.

[126] F.-S. Choa, "High-efficiency directly modulated laser links," in Proc. Int. Topical Meeting Microwave Photonics MWP '01, 2002.

[127] J. Cai, Z. Chen, X. Ji, Y. Sun, J. Lin, X. Zhao, X. Yu, J. Zhang, J. Yan, M. Raj, and F.-S. Choa, "Fast frequency tuning characteristics of a FM laser based on the integrated MQW DFB laser/EA modulator device," in Proc. 16th Annual Meeting of the IEEE Lasers and Electro-Optics Society LEOS 2003, vol. 2, 2003, pp. 638-639.

[128] T. Okoshi, K. Kikuchi, and A. Nakayama, "Novel method for high resolution measurement of laser output spectrum," Electronics Letters, vol. 16, no. 16, pp. $630-631$, Jul 1980. 